University of Tennessee Health Science Center

UTHSC Digital Commons

\title{
$5-2009$
}

\section{Distinct DNA Damage Signaling in the Brain Distinguishes ATLD, NBS, and ATR-Seckel Syndrome}

\author{
Erin Renee Phillips Shull \\ University of Tennessee Health Science Center
}

Follow this and additional works at: https://dc.uthsc.edu/dissertations

Part of the Neurosciences Commons

\section{Recommended Citation}

Shull, Erin Renee Phillips, "Distinct DNA Damage Signaling in the Brain Distinguishes ATLD, NBS, and ATRSeckel Syndrome" (2009). Theses and Dissertations (ETD). Paper 242. http://dx.doi.org/10.21007/ etd.cghs.2009.0290.

This Dissertation is brought to you for free and open access by the College of Graduate Health Sciences at UTHSC Digital Commons. It has been accepted for inclusion in Theses and Dissertations (ETD) by an authorized administrator of UTHSC Digital Commons. For more information, please contact jwelch30@uthsc.edu. 


\title{
Distinct DNA Damage Signaling in the Brain Distinguishes ATLD, NBS, and ATR- Seckel Syndrome
}

\author{
Abstract \\ DNA double strand breaks create a situation of extreme stress under which a cell must either be capable \\ of repairing the lesions in order to continue replication or succumb to death. Not surprisingly, deficiencies \\ in DNA repair genes often lead to human diseases frequently associated with genomic instability, cancer \\ proneness, and neuropathology. Neurological consequences of aberrant DNA repair mechanisms vary \\ depending upon the affected gene and the pathway in which it operates. Ataxia-telangiectasia (A-T) is the \\ prototypical disease associated with DNA double strand break (DSB) repair deficiency and is \\ characterized by severe neural pathology. A-T results from homozygous mutations that inactivate the \\ ataxia-telangiectasia mutated (ATM) gene, and typically presents in early childhood as cerebellar ataxia \\ accompanied by ocular telangiectasias, elevated $\alpha$-fetoprotein levels, and lymphoma predisposition \\ compounded by radiosensitivity. The ATM protein is a large $(\sim 380 \mathrm{kDa})$ protein kinase within the PI3K \\ family, which mediates cell cycle checkpoint activity and induces apoptosis through phosphorylation of \\ copious substrates. It is thought to be through apoptotic induction of newly post-mitotic cells harboring \\ lethal levels of DNA damage that Atm maintains integrity of the CNS, as it prevents incorporation of \\ genomically compromised neurons into the mature nervous system. Hypomorphic mutations in the \\ Mre11/Rad50/NBS1 complex (MRN complex), which is required for efficient ATM activity, result in distinct \\ syndromes. Ataxia-telangiectasia-like disease (ATLD) results from truncating mutations in Mre11 and \\ presents with neurodegeneration similar to that observed in $A-T$, but with longer latency and less severity. \\ Mutations in the FHA and BRCT domains of NBS1 result in Nijmegen Breakage Syndrome (NBS), which is \\ characterized by microcephaly without evidence of neurodegeneration. Thus far mutations in Rad50 have \\ not been reported in humans. In order to better understand the roles of ATM and MRN signaling in \\ maintaining physical and functional integrity of the CNS, mutant mice either null for Atm or carrying \\ hypomorphic mutations in Mre11 or Nbs1 were subjected to either endogenous or exogenous forms of \\ DNA damage and the signaling response was assessed in the brain. To understand the contrasting \\ neuropathology resulting from Mre11 or Nbs1 hypomorphic mutations, we analyzed neural tissue from \\ Mre11ATLD1/ATLD1 and Nbs1DB/DB mice after genotoxic stress. We found a pronounced resistance to \\ DNA damage-induced apoptosis after ionizing radiation or DNA ligase IV (Lig4) loss in the Mre11ATLD1/ \\ ATLD1 nervous system that was associated with defective Atm activation and phosphorylation of its \\ substrate $\mathrm{p} 53$. Apoptosis occurred normally in the $\mathrm{Nbs} 1 \Delta \mathrm{B} / \Delta \mathrm{B}$ brain. We also conditionally disrupted Lig4 \\ throughout the nervous system using Nestin-cre (Lig4Nes-Cre), and while viable, these mice showed \\ pronounced microcephaly and a prominent age-related accumulation of DNA damage throughout the \\ brain. Either Atm-/- or Mre11ATLD1/ATLD1 genetic backgrounds, but not Nbs1 $\Delta B / \triangle B$ rescued Lig4Nes- \\ Cre microcephaly. Thus, DNA damage signaling in the nervous system is different between ATLD and \\ NBS, and likely explains their respective neuropathology. Ataxia-telangiectasia and Rad3-related (ATR) is a \\ large serine/threonine kinase that is highly similar in structure to ATM and phosphorylates many \\ overlapping substrates. Hypomorphic mutations in ATR have been associated with some cases of Seckel \\ syndrome, a microcephalic condition. Despite the similarities between ATM and ATR, inactivation of either \\ kinase has quite distinct consequences. Unlike ATM, ATR is essential for embryonic development, as Atr- \\ /- embryos are lethal by E7.5. To better understand the differing roles of ATM and ATR in the nervous \\ system, a conditional Atr mutant was crossed onto the Nestin-cre transgenic line. AtrloxP/loxP; Nestin-cre \\ ( AtrNes-cre) animals displayed early post-natal lethality and severe neurogenesis defects, particularly \\ striking in the cerebellum due to decreased proliferation and viability of granule neuron progenitors. \\ Surprisingly, progenitors in the rhombic lip were largely unaffected in AtrNes-cre embryos whereas the \\ granule cell precursors of the cerebellar external germinal layer (EGL) displayed DNA damage as early as \\ E13.5 and p53-dependant apoptosis by E15.5. Between E15.5 and E16.5 p53 independent cell cycle arrest \\ was inferred by decreased proliferation of the remaining granule cell precursors in the EGL. DNA damage
}


was not observed in the rhombic lip until E16.5. The forebrain showed a similar pattern of acquired damage followed by apoptosis and decreased proliferation. The p53-null genetic background did not substantially rescue the AtrNes-cre phenotype despite blocking apoptosis. This data indicates that proliferation arrest is the major factor causing ATR-Seckel syndrome and that ATR does not significantly contribute to p53-induced apoptosis in proliferating neural progenitors. AtrNes-cre animals were crossed onto an Atm conditional background (AtmNes-cre) in order to assess how ATM and ATR cooperate in maintaining homeostasis in the brain. The AtmNes-cre background failed to alter the AtrNes-cre phenotype, underscoring the divergence of ATR and ATM function during embryogenesis, despite overlapping substrates between the kinases. In summary, this work provides us with important insight into the requirements for specific DNA damage signaling molecules in the nervous system. ATM and ATR appear to have distinct roles in the nervous system with ATM required for elimination of compromised early post-mitotic neurons, while ATR is critical for maintaining integrity and replicative capacity of progenitor populations. Based upon this study, there is no obvious cross-talk or overlap between Atm and Atr signaling in the nervous system as each plays a separate role in different cell populations.

Furthermore, we have begun to tease out the existence of independent signaling roles between Mre11 and Nbs1 in regard to Atm activity. While Mre11 mutations associated with ATLD compromise Atmdependant apoptosis, Nbs1 hypomorphism linked to NBS does not appear to negatively impact upon known Atm function in the nervous system. Overall these data provide new insights into the specific genetic requirements for DNA double strand break repair in the nervous system.

\section{Document Type}

Dissertation

Degree Name

Doctor of Philosophy (PhD)

\section{Program}

Interdisciplinary Program

\section{Research Advisor}

Peter J. McKinnon, Ph.D.

\section{Keywords}

ATM, ATR, brain, DNA damage, DNA repair, MRN complex

\section{Subject Categories}

Medical Sciences | Medicine and Health Sciences | Neurosciences 


\title{
Distinct DNA Damage Signaling in the Brain Distinguishes ATLD, NBS, and ATR-Seckel Syndrome
}

\author{
A Dissertation \\ Presented for \\ The Graduate Studies Council \\ The University of Tennessee \\ Health Science Center
}

In Partial Fulfillment

Of the Requirements for the Degree

Doctor of Philosophy

From The University of Tennessee

By Erin Renee Phillips Shull

May, 2009 
Portions of Chapter 1 (C) 2007 by Nature Publishing Group Portions of Chapter 2 (c) 2009 by Cold Spring Harbor Laboratory Press All other material (C) 2008 by Erin R. P. Shull

\author{
All rights reserved
}




\section{DEDICATION}

To my Herbert, whose sturdy character and unwavering dedication to me made it possible to tolerate the sacrifice that the completion of this work required and which will help sustain us both in pursuing whatever endeavors follow.

I also dedicate this work to my Mom, who endowed me with will and perseverance, and to my brothers James and Brian who keep me grounded. 


\section{ACKNOWLEDGMENTS}

I thank my mentor Dr. Peter McKinnon for having granted me the honor of being the first graduate student to complete the Ph.D. process under his guidance and for his support during this process. Having such a kind and decent "boss" always makes life easier. I extend my eternal gratitude to Dr. Youngsoo Lee, who helped me through every aspect of this experience. I consider Youngsoo my second mentor. Dr. Helen Russell provided me with everything from technical support (particularly for generating the conditional Lig4 mice and greatly assisting in generating the conditional Atm mice) to the occasional home-cooked meal with her family. Dr. Pierre-Olivier Frappart initiated the Atr project. I would also like to acknowledge Jingfeng Zhao for providing some of the data shown in Chapter 2, and every other member of the McKinnon lab for his/her support over the years.

Dr. John Petrini provided the Mre $11^{A T L D}$ and the $N b s 1^{\triangle B}$ mice, and Dr. Eric Brown gave us the $A t r^{\text {loxP/loxP }}$ mice. Without these animal models, none of this work could have been done. Dr. Chris Calabrese of the St. Jude Children's Research Hospital Animal Imaging Facility performed CT scans of the Lig $4^{\text {Nes-cre }}$ spinal column.

I extend my gratitude to my committee members Dr. Larry Pfeffer, Dr. Susan Senogles, Dr. Rich Smeyne, and Dr. Jian Zuo. The graduate committee is an integral aspect of the Ph.D. process, and I deeply appreciate the time taken from their own schedules to offer input and guidance toward my work. I also thank Dr. Suzanne Baker, who was generous enough to read and critique my "grant" for the admission to candidacy exam.

This work was supported by the National Institutes of Health (NS-37956 and CA21765) and the CCSG (P30 CA21765), and the American Lebanese and Syrian Associated Charities of St. Jude Children's Research Hospital. 


\begin{abstract}
DNA double strand breaks create a situation of extreme stress under which a cell must either be capable of repairing the lesions in order to continue replication or succumb to death. Not surprisingly, deficiencies in DNA repair genes often lead to human diseases frequently associated with genomic instability, cancer proneness, and neuropathology. Neurological consequences of aberrant DNA repair mechanisms vary depending upon the affected gene and the pathway in which it operates. Ataxia-telangiectasia $(\mathrm{A}-\mathrm{T})$ is the prototypical disease associated with DNA double strand break (DSB) repair deficiency and is characterized by severe neural pathology. A-T results from homozygous mutations that inactivate the ataxia-telangiectasia mutated (ATM) gene, and typically presents in early childhood as cerebellar ataxia accompanied by ocular telangiectasias, elevated $\alpha$ fetoprotein levels, and lymphoma predisposition compounded by radiosensitivity. The ATM protein is a large $(\sim 380 \mathrm{kDa})$ protein kinase within the PI3K family, which mediates cell cycle checkpoint activity and induces apoptosis through phosphorylation of copious substrates. It is thought to be through apoptotic induction of newly post-mitotic cells harboring lethal levels of DNA damage that Atm maintains integrity of the CNS, as it prevents incorporation of genomically compromised neurons into the mature nervous system. Hypomorphic mutations in the Mre11/Rad50/NBS1 complex (MRN complex), which is required for efficient ATM activity, result in distinct syndromes. Ataxiatelangiectasia-like disease (ATLD) results from truncating mutations in Mre11 and presents with neurodegeneration similar to that observed in A-T, but with longer latency and less severity. Mutations in the FHA and BRCT domains of NBS1 result in Nijmegen Breakage Syndrome (NBS), which is characterized by microcephaly without evidence of neurodegeneration. Thus far mutations in Rad50 have not been reported in humans.

In order to better understand the roles of ATM and MRN signaling in maintaining physical and functional integrity of the CNS, mutant mice either null for Atm or carrying hypomorphic mutations in Mre11 or Nbs1 were subjected to either endogenous or exogenous forms of DNA damage and the signaling response was assessed in the brain. To understand the contrasting neuropathology resulting from Mre11 or Nbs1 hypomorphic mutations, we analyzed neural tissue from Mre $11^{A T L D 1 / A T L D 1}$ and $\mathrm{Nbs} 1^{\triangle B / \triangle B}$ mice after genotoxic stress. We found a pronounced resistance to DNA damage-induced apoptosis after ionizing radiation or DNA ligase IV (Lig4) loss in the Mre 1 $1^{\text {ATLDI/ATLD1 }}$ nervous system that was associated with defective Atm activation and phosphorylation of its substrate $\mathrm{p} 53$. Apoptosis occurred normally in the $N b s 1^{\Delta B / \Delta B}$ brain. We also conditionally disrupted Lig4 throughout the nervous system using Nestin-cre ( $\left.\mathrm{Lig} 4^{\mathrm{Nes}-\mathrm{Cre}}\right)$, and while viable, these mice showed pronounced microcephaly and a prominent agerelated accumulation of DNA damage throughout the brain. Either $\mathrm{Atm}^{-/-}$or Mre $11^{A T L D 1 / A T L D 1}$ genetic backgrounds, but not $N b s 1^{\triangle B / \triangle B}$ rescued Lig $4^{\text {Nes-Cre }}$ microcephaly. Thus, DNA damage signaling in the nervous system is different between ATLD and NBS, and likely explains their respective neuropathology.
\end{abstract}

Ataxia-telangiectasia and Rad3-related (ATR) is a large serine/threonine kinase that is highly similar in structure to ATM and phosphorylates many overlapping 
substrates. Hypomorphic mutations in ATR have been associated with some cases of Seckel syndrome, a microcephalic condition. Despite the similarities between ATM and ATR, inactivation of either kinase has quite distinct consequences. Unlike ATM, ATR is essential for embryonic development, as Atr $^{-/}$embryos are lethal by E7.5. To better understand the differing roles of ATM and ATR in the nervous system, a conditional Atr mutant was crossed onto the Nestin-cre transgenic line. Atr ${ }^{\text {loxP/loxP }} ;$ Nestin-cre $\left(\right.$ Atr $^{\text {Nes-cre }}$ ) animals displayed early post-natal lethality and severe neurogenesis defects, particularly striking in the cerebellum due to decreased proliferation and viability of granule neuron progenitors. Surprisingly, progenitors in the rhombic lip were largely unaffected in $\mathrm{Atr}^{\mathrm{Nes}-}$ cre embryos whereas the granule cell precursors of the cerebellar external germinal layer (EGL) displayed DNA damage as early as E13.5 and p53-dependant apoptosis by E15.5. Between E15.5 and E16.5 p53 independent cell cycle arrest was inferred by decreased proliferation of the remaining granule cell precursors in the EGL. DNA damage was not observed in the rhombic lip until E16.5. The forebrain showed a similar pattern of acquired damage followed by apoptosis and decreased proliferation. The p53-null genetic background did not substantially rescue the Atr $^{\text {Nes-cre }}$ phenotype despite blocking apoptosis. This data indicates that proliferation arrest is the major factor causing ATRSeckel syndrome and that ATR does not significantly contribute to p53-induced apoptosis in proliferating neural progenitors. Atr ${ }^{\text {Nes-cre }}$ animals were crossed onto an Atm conditional background ( Atm $^{\text {Nes-cre }}$ ) in order to assess how ATM and ATR cooperate in maintaining homeostasis in the brain. The $\mathrm{Atm}^{\text {Nes-cre }}$ background failed to alter the Atr ${ }^{\text {Nes- }}$ cre phenotype, underscoring the divergence of ATR and ATM function during embryogenesis, despite overlapping substrates between the kinases.

In summary, this work provides us with important insight into the requirements for specific DNA damage signaling molecules in the nervous system. ATM and ATR appear to have distinct roles in the nervous system with ATM required for elimination of compromised early post-mitotic neurons, while ATR is critical for maintaining integrity and replicative capacity of progenitor populations. Based upon this study, there is no obvious cross-talk or overlap between Atm and Atr signaling in the nervous system as each plays a separate role in different cell populations. Furthermore, we have begun to tease out the existence of independent signaling roles between Mre11 and Nbs1 in regard to Atm activity. While Mre11 mutations associated with ATLD compromise Atmdependant apoptosis, Nbs1 hypomorphism linked to NBS does not appear to negatively impact upon known Atm function in the nervous system. Overall these data provide new insights into the specific genetic requirements for DNA double strand break repair in the nervous system. 


\section{TABLE OF CONTENTS}

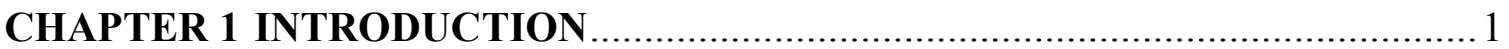

1.1 DNA Double Strand Break Repair................................................................. 1

1.2 Consequence of DSB Repair Deficiencies in Humans.................................... 3

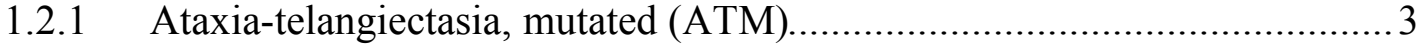

1.2.2 Ataxia-telangiectasia and Rad3-related (ATR) ...................................... 4

1.2.3 DNA ligase IV (Lig4) ...................................................................... 5

1.2.4 The Mre11/Rad50/Nbs1 complex (MRN) ............................................. 5

1.2.5 Mouse models of DNA repair deficiencies .......................................... 6

CHAPTER 2 DIFFERENTIAL DNA DAMAGE SIGNALING ACCOUNTS FOR DISTINCT NEUROPATHOLOGY ASSOCIATED WITH

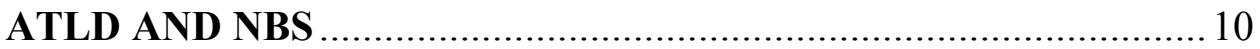

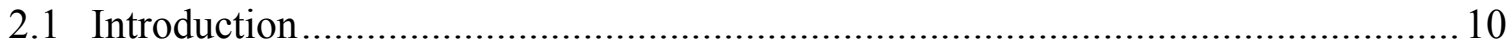

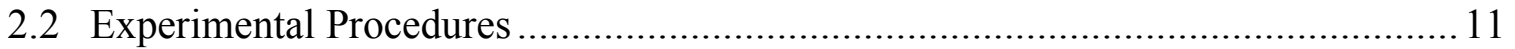

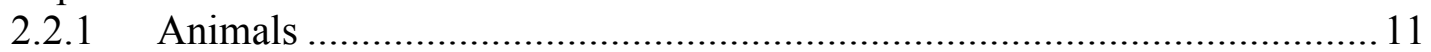

2.2.2 Histology and immunohistochemistry ............................................ 12

2.2.3 Astrocyte culture....................................................................... 12

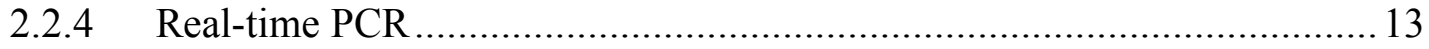

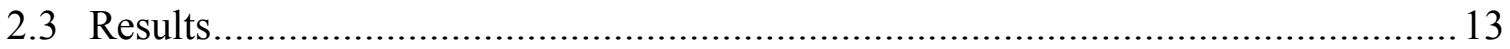

2.3.1 Kinetics of H2AX phosphorylation in Atm, Mre11, Nbs1, or

Lig4-deficient astrocytes ........................................................... 13

2.3.2 IR-induced apoptosis is defective in the Mre $11^{\text {ATLDI/ATLDI }}$ brain ................. 14

2.3.3 Characterization of $\mathrm{Lig} 4^{\text {Nes-cre }}$ mice ..................................................... 21

2.3.4 Microcephaly after Lig4 loss requires Atm signaling............................29

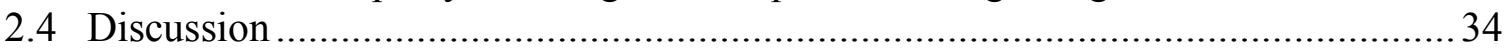

CHAPTER 3 ATR IS REQUIRED FOR GENOMIC INTEGRITY AND PROLIFERATION OF NEURAL PROGENITORS ....................... 36

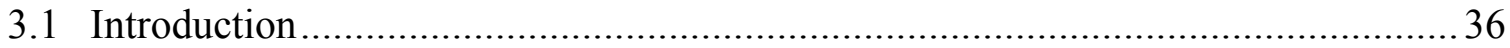

3.2 Experimental Procedures .......................................................................... 42

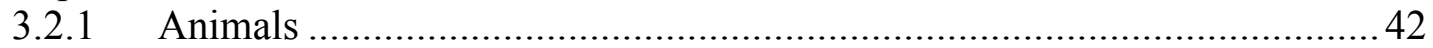

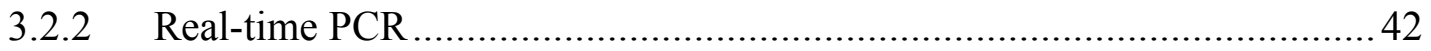

3.2.3 Histology and immunohistochemistry .......................................... 43

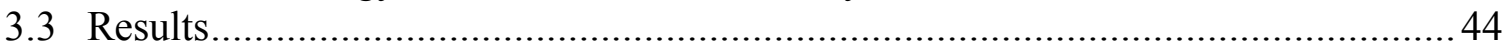

3.3.1 Atr deficiency results in microcephaly and cerebellar dysgenesis ............. 44

3.3.2 Atr loss was associated with increased DNA damage and p53-dependent apoptosis in the developing brain .................................................. 47

3.3.3 Atr deficiency results in depletion of proliferating cell populations during neurogenesis ............................................................. 58

3.3.4 Atm and Atm functions are not obviously redundant in the developing

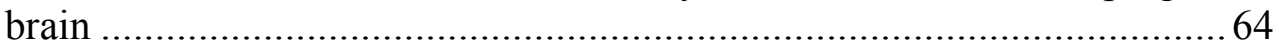

3.4 Discussion 
CHAPTER 4 DISCUSSION

4.1 Apoptosis Is Central to the Manifestation of Neuropathology Associated with DSB Repair Deficiencies

4.2 The Impact of DNA Damage Signaling upon Cell Cycle Regulation in Neural

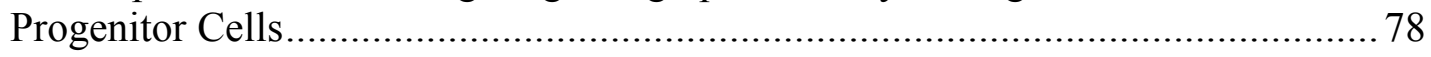

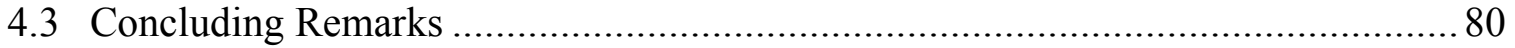

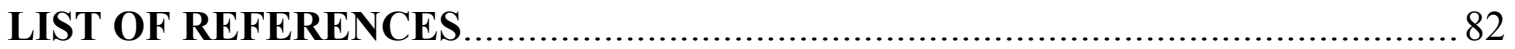

VITA 


\section{LIST OF FIGURES}

Figure 1.1 Diseases associated with DNA double-strand break repair deficits.

Figure 2.1 Kinetics of H2AX phosphorylation in $\mathrm{Lig}^{\mathrm{Nes}-\mathrm{cre}}, \mathrm{Atm}^{-/}$,

Mre $11^{A T L D 1 / A T L D 1}$, and $N b s 1^{\triangle B / \triangle B}$ astrocytes.

Figure 2.2 DNA DSB repair kinetics in Lig4 ${ }^{\text {Nes-cre }}, \mathrm{Atm}^{-/-}$, Mrel $1^{\text {ATLD1/ATLD1 }}$, and

$N b s 1^{\Delta B / \Delta B}$ astrocytes. ............................................................. 16

Figure 2.3 Radiation-induced apoptosis in Mre $11^{A T L D I / A T L D 1}$ and $N b s 1^{\triangle B / \triangle B}$ CNS.... 18

Figure 2.4 DNA damage responses in the Mre $11^{A T L D 1 / A T L D 1}$ and $N b s 1^{\triangle B / \triangle B}$ nervous

systems.

Figure 2.5 Real-time PCR demonstrating Lig4 gene excision in E13.5 Lig4 ${ }^{\text {Nes-cre }}$

CNS.

Figure 2.6 Diminished cerebellar size reflective of overall microcephaly in Lig $4^{\text {Nes-cre }}$ mice.

Figure 2.7 CT scan of spinal column collected from $\operatorname{Lig} 4^{\text {Nes-cre }}$ mouse with end-stage hindlimb ataxia and age-matched $\operatorname{Lig} 4^{\mathrm{Ctrl}}$.

Figure 2.8 Histology observed in Lig $4^{\text {Nes-cre }}$ spinal cord...................................... 25

Figure 2.9 Pattern of 53BP1 foci in E15.5 Lig $4^{\text {Nes-cre }}$ brain. ................................. 26

Figure 2.10 Comparison of 53BP1 foci in caudate putamen of P5, 4 month old, and 12 month old Lig4 ${ }^{\text {Nes-cre }}$ mice.

Figure 2.11 Comparison of $\gamma-\mathrm{H} 2 \mathrm{AX}$ expression in cerebella of $\mathrm{P} 4,4$ month old, and 12 month old Lig $4^{\text {Nes-cre }}$ mice.

Figure 2.12 Spatiotemporal mapping of 53BP1 and H2AX foci in Lig $4^{\text {Nes-cre }}$ brains. . 30

Figure 2.13 $\gamma$-H2AX foci persist in irradiated Lig $4^{\text {Nes-cre }}$ brain.

Figure 2.14 Mre11 hypomorphism and Atm loss partially rescue microcephaly in Lig4 ${ }^{\text {Nes-cre }}$ mice.

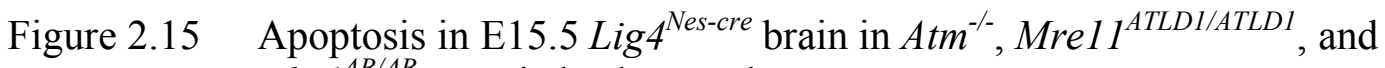
$N b s 1^{\Delta B / \Delta B}$ genetic backgrounds.

Figure 3.1 Activation and signaling pathways of ATR and ATM. .38 
Figure 3.2 Dramatic changes in cerebellar morphology that occur between embryogenesis and adulthood..

Figure 3.3 Depiction of post-natal cerebellar development

Figure 3.4 Nestin-cre mediated deletion efficiency of floxed Atr region in E13.5 Atr ${ }^{\text {Nes-cre }}$ CNS.

Figure 3.5 Atr ${ }^{\text {Nes-cre }}$ mice showed severe growth defects accompanied by microcephaly and neurogenesis defects.

Figure 3.6 Nissl stained coronal sections of the P5 Atr ${ }^{\text {Nes-cre }}$ olfactory bulb and rostral migratory stream.

Figure $3.7 \quad$ P5 Atr ${ }^{\text {Nes-cre }}$ forebrain stained for Nissl substance.

Figure 3.8 Pattern of $\gamma-\mathrm{H} 2 \mathrm{AX}$ foci in the $A t r^{\text {Nes-cre }}$ cerebellum.

Figure 3.9 Pattern of $\gamma-\mathrm{H} 2 \mathrm{AX}$ in the ganglionic eminence and lateral ventricular zone of embryonic Atr $^{\text {Nes-cre }}$ mice.

Figure 3.10 Pattern of $\gamma$-H2AX formation in the developing Atr $^{\text {Nes-cre }}$ cortex. .53

Figure 3.11 Pattern of cell death in the Atr $^{\text {Nes-cre }}$ cerebellum.

Figure 3.12 Pattern of cell death in lateral ventricular zone and ganglionic eminence of $A t r^{\text {Nes-cre }}$ embryos.

Figure 3.13 Pattern of cell death in the ventricular zone of the embryonic Atr ${ }^{\text {Nes-cre }}$ cortex.

Figure 3.14 Immunostaining against p53-phosphoserine 18 in E16.5 $\mathrm{Atr}^{\mathrm{Ctrl}}$ and Atr $^{\text {Nes-cre }}$ samples.

Figure 3.15 Apoptosis was rescued in $A t r^{\text {Nes-cre }} p 53^{-/-}$animals, but neuropathology was not affected by p53 loss in P5 Atr ${ }^{\text {Nes-cre }}$ mice.

Figure 3.16 Proliferation of the EGL in the E16.5 Atr ${ }^{\text {Nes-cre }}$ cerebellum is decreased.. 61

Figure 3.17 The rhombic lip is largely unaffected in $A t r^{\text {Nes-cre }}$ embryos. ....................62

Figure 3.18 Immunostaining against PCNA in E18.5 Atr $^{\text {Nes-cre }}$ forebrain. .................. 63

Figure 3.19 Immunostaining against PCNA in P5 Atr ${ }^{\mathrm{Ctrl}}$ and $\mathrm{Atr}^{\text {Nes-cre }}$ sagittal sections of cerebellum and forebrain. 
Figure 3.20 Efficient Nestin-cre mediated excision of Atm exon 58 and confirmation of genotype consistent with $\mathrm{Atm}^{-/-}$mice. .66

Figure $3.21 \mathrm{Atr}^{\text {Nes-cre }} \mathrm{Atm}^{\text {Nes-cre }}$ mice are indistinguishable from $\mathrm{Atr}^{\text {Nes-cre }}$ littermates.. 68

Figure 3.22 DNA damage signaling in the E16.5 $\mathrm{Atr}^{\text {Nes-cre }} \mathrm{Atm}^{\text {Nes-cre }}$ EGL.................69 


\section{LIST OF ABBREVIATIONS}

A-T

ATLD

ATM

ATM

Atm

Atm

Atm $^{\text {Ctrl }}$

Atm $^{\text {Nes-cre }}$

ATR

ATR

Atr

Atr

Atr ${ }^{C t r l}$

ATRIP

Atr ${ }^{\text {Nes-cre }}$

ATR-SS

BG

BrdU

$\mathrm{CC}$

CDK

$\mathrm{CP}$

CTX

DDR

DG

DSB

EGL

E0-18.5

GE

GP

$\gamma$-H2AX

$\mathrm{HR}$

IGL

IR

$\mathrm{kDa}$

LIG4

LIG4

Lig4
Ataxia-telangiectasia

Ataxia-telangiectasia like disease

Ataxia-telangiectasia mutated, human protein

Ataxia-telangiectasia mutated, human gene

Ataxia-telangiectasia mutated, mouse protein Ataxia-telangiectasia mutated, mouse gene

$\mathrm{Atm}^{+/ l o x P}$ or $\mathrm{Atm}^{+/+}$(+ or - Nestin-cre $)$, or Atm ${ }^{\operatorname{lox} P / l o x P}$

Atm $^{\text {loxP/loxP }} ;$ Nestin-cre

Ataxia-telangiectasia and Rad 3 related, human protein

Ataxia-telangiectasia and Rad 3 related, human gene

Ataxia-telangiectasia and $\mathrm{Rad} 3$ related, mouse protein

Ataxia-telangiectasia and $\mathrm{Rad} 3$ related, mouse gene

$A_{t r}^{+/ l o x P}$ or $A_{t r}^{+/+}$(+ or - Nestin-cre $)$, or Atr ${ }^{\operatorname{lox} P / l o x P}$

ATR interacting protein

Atr $^{\text {loxP/loxP }}$; Nestin-cre

ATR-Seckel syndrome

Basal ganglia

5-Bromo-2'-deoxyuridine

Corpus callosum

Cyclin dependent kinase

Caudate putamen

Cortex

DNA damage response

Dentate gyrus

DNA double strand break

External granule layer

Embryonic day of gestation 0-18.5

Ganglionic eminence

Globus pallidus

H2AX-phospho-ser139

Homologous recombination

Internal granule layer

Ionizing radiation

kilo Dalton

DNA ligase IV, human protein

DNA ligase IV, human gene

DNA Ligase IV, mouse protein 


\begin{tabular}{|c|c|}
\hline Lig4 & DNA ligase IV, mouse gene \\
\hline $\operatorname{Lig} 4^{C t r l}$ & $\begin{array}{l}\text { Lig4 }{ }^{+/ l o x P} \text { or Lig4 } 4^{+/+}(+ \text {or }- \text { Nestin-cre }) \text {, or } \\
\text { Lig } 4^{\text {loxP/loxP }}\end{array}$ \\
\hline Lig $4^{\text {Nes-Cre }}$ & Lig ${ }^{\text {loxP/loxP }} ;$ Nestin-cre \\
\hline MRE11 & Meiotic recombination 11 , human protein \\
\hline Mre11 & Meiotic recombination 11 , mouse protein \\
\hline Mre11 & Meiotic recombination 11 , mouse gene \\
\hline $\mathrm{MRN}$ & Mre11/RAD50/NBS1 complex \\
\hline NBS & Nijmegen breakage syndrome \\
\hline NBS1 & Nibrin (p95), human protein \\
\hline Nbs 1 & Nibrin (p95), mouse protein \\
\hline$N b s 1$ & Nibrin (p95), mouse gene \\
\hline NHEJ & Non-homologous end-joining \\
\hline $\mathrm{OB}$ & Olfactory bulb \\
\hline OMIM & Online Mendelian inheritance of man \\
\hline $\mathrm{PC}$ & Purkinje cells \\
\hline PCNA & Proliferating cell nuclear antigen \\
\hline PFA & Paraformaldehyde \\
\hline $\mathrm{P} 0-21$ & Post-natal day $0-21$ \\
\hline RL & Rhombic lip \\
\hline RPA & Replication protein A \\
\hline SSB & DNA single strand break \\
\hline TopBP1 & Topoisomerase (DNA) II binding protein \\
\hline $\mathrm{VZ}$ & Ventricular zone \\
\hline V4 & Fourth ventricle \\
\hline 911 & RAD9-RAD1-HUS1 \\
\hline
\end{tabular}




\section{CHAPTER 1 INTRODUCTION*}

\subsection{DNA Double Strand Break Repair}

DNA double strand breaks (DSBs) are considered the most deleterious form of DNA damage. When left unrepaired, DSBs can cause apoptosis or the accumulation of genomic instability. DNA damage initiates complex signaling cascades that result in cell cycle checkpoint activation, DNA repair or apoptosis. Much of our understanding of these pathways comes from studies using patient-derived cell lines or mutant mice designed to model DNA repair deficits (Friedberg \& Meira, 2006; Rosenthal \& Brown, 2007). ATM and ATR sit at the apex of DNA double strand break signaling, and are responsible for phosphorylating multiple substrates that impact cellular fate after damage. While ATM is predominantly activated by DSBs such as those produced by irradiation and subsequently initiates cell cycle checkpoints and/or apoptosis, ATR responds to agents that interfere with DNA replication (i.e., UV irradiation and hydroxyurea) and is essential to cellular viability (Brown \& Baltimore, 2000; Cimprich \& Cortez, 2008; Hurley \& Bunz, 2007). The precise recruitment order of DNA damage signaling molecules to DSB sites is unclear, as the rapidity with which the damage response is activated makes delineating what comes first challenging. Phosphorylation of H2AX on the C-terminus Ser 139 is one of the earliest events occurring in response to DNA damage, and can be visualized immunohistochemically as distinct nuclear foci that mark DNA DSBs. A major function of $\gamma-\mathrm{H} 2 \mathrm{AX}$ is recruitment of other DNA damage response factors to the sites of DNA DSBs (Fernandez-Capetillo et al., 2004; Sedelnikova et al., 2003). Though H2AX is not essential in the mouse, its loss predisposes to genomic instability (Celeste et al., 2003; Celeste et al., 2002). H2AX phosphorylation facilitates recruitment of MDC1 to the DNA break via binding to the $\gamma-\mathrm{H} 2 \mathrm{AX}$ C-terminus (Goldberg et al., 2003; Lou et al., 2003; Stewart et al., 2003). This interaction is required for signal amplification and effective modulation of downstream signaling (BekkerJensen et al., 2005; Lou et al., 2006; Stucki et al., 2005; Stucki \& Jackson, 2006).

Two predominant pathways of DSB repair have been identified, nonhomologous end-joining (NHEJ) and homologous recombination (HR). These pathways are differentially required depending upon developmental stages and tissue type. A distinguishing characteristic between the DNA DSB pathways is the requirement of HR for a sister chromatid present in the S/G2 phase of the cell cycle. This implies that the repair of DSBs in non-replicating cells will occur via NHEJ, and it has been shown in the developing murine nervous system that abrogation of HR results in Atm-independent apoptosis in proliferating regions whereas loss of NHEJ repair leads to Atm-dependant apoptosis in post-mitotic areas (Gao et al., 2000; Lee et al., 2000; Orii et al., 2006). HR is an error-free process that uses a sister chromatid as template DNA to achieve precise repair (Helleday et al., 2007; Thacker \& Zdzienicka, 2004; West, 2003). Homology* Adapted by permission from Phillips ER and McKinnon PJ (2007). Oncogene, 26,
7799-808. 
directed repair initially involves an Mre11-Rad50-Nbs1 (MRN)-mediated 5'-3' resection followed by Rad51-mediated homology search and strand invasion. The Rad51 recombinase complex functions in concert with a series of other factors including the Rad51 paralogs (Xrcc2, Xrcc3, RAD51L1, RAD51L2, RAD51L3), Rad52, and Rad54 proteins during which the processed DNA strand invades the sister chromatid and initiates recombination (Helleday et al., 2007; Heyer et al., 2006; Thacker and Zdzienicka, 2004; West, 2003; Wyman et al., 2004). Strand invasion and migration involves formation of a structure termed a Holliday Junction, which is resolved via Rad54, Mus81/Emc1 and Rad51C/Xrcc3 following repair synthesis by DNA polymerase, thus completing the repair process. As would be surmised from the essential importance of homologous recombination during embryonic development, studies in the mouse indicate that many of the above factors are essential for survival and their inactivation leads to lethality (Friedberg \& Meira, 2006).

In contrast to HR, NHEJ is an error-prone mechanism that involves the DNAdependent protein kinase (DNA-PK) and the DNA ligaseIV (Lig4)-Xrcc4 complex that together facilitate re-joining of broken non-compatible DNA ends (Lieber et al., 2003). The DNA-PK complex contains the Ku70 and Ku80 proteins, which recognize the break and mediate recruitment of the DNA-PK catalytic subunit (DNA-PKcs) a serine/threonine kinase whose autophosphorylation and phosphorylation of other factors including H2AX is required for efficient NHEJ (Bassing \& Alt, 2004; Burma \& Chen, 2004; Lees-Miller \& Meek, 2003). Ligation of the DNA ends occurs via Lig4 in conjunction with its binding partner Xrcc4. An additional factor, Cernunnos/XLF, has been identified as a binding partner of the Lig4-Xrcc4 complex and appears to be necessary for efficient ligation via NHEJ (Ahnesorg et al., 2006; Buck et al., 2006). Subsets of NHEJ may involve other factors such as Artemis (Jeggo \& O'Neill, 2002; Moshous et al., 2001). The extent to which Artemis is required during canonical NHEJ (independent of $\mathrm{V}(\mathrm{D}) \mathrm{J}$ recombination) remains uncertain, as conflicting data on its requirement for end-joining have been reported (Riballo et al., 2004; Rooney et al., 2003; Wang et al., 2005c; Zhang et al., 2004). However, DNA Ligase IV (Lig4) and Xrcc4 are essential for NHEJ and embryonic survival (Barnes et al., 1998; Frank et al., 1998; Gao et al., 1998), and their interaction is required for efficient end-joining as Lig4 alone is not sufficient to complete repair in the absence of Xrcc4 (Bassing \& Alt, 2004; Critchlow et al., 1997; Grawunder et al., 1997; Grawunder et al., 1998a; Grawunder et al., 1998b). While HR is available for repair during late $\mathrm{S}$ phase, NHEJ is most prominently activated in G1, G0, and early S phase of the cell cycle (Rothkamm et al., 2003; Thacker \& Zdzienicka, 2004). However, animal studies indicate that there is cooperativity between these pathways during development (Couedel et al., 2004; Mills et al., 2004).

While NHEJ requires DNA-PK and Lig4-Xrcc4, studies suggest that there may be an NHEJ repair pathway that utilizes other components, and in some cases can occur without DNA-PK (DiBiase et al., 2000; Perrault et al., 2004). For instance DNA ligase III (Lig3) has been implicated as an alternate ligase that may function in NHEJ (Wang et al., 2005; Windhofer et al., 2007). In vitro evidence implicating PARP-1 and Xrcc1/Lig3 in NHEJ has also been obtained through end-joining assays (Audebert et al., 2004). The physiologic relevance of such phenomena remains unclear though, as no substantial 
compensatory mechanisms appear in either Xrcc4-deficient or Lig4-deficient mice. If Lig3 does compensate in vivo, it does so only partially and is insufficient for normal mammalian development in the absence of Lig4.

The requirement for efficient DNA repair mechanisms is underscored by the severe consequences observed in individuals lacking full activity of signaling factors or repair genes. Human deficiencies in ATM, ATR, MRN, or Lig4 (to list only a few) have been identified and present with neuropathology, immunodeficiency, and chromosomal instability. Though many characteristics of such genetic aberrations overlap, there are also distinctions indicative of differing specific functions.

\subsection{Consequence of DSB Repair Deficiencies in Humans}

\subsubsection{Ataxia-telangiectasia, mutated (ATM)}

The canonical example of DNA DSB sensitivity in humans is the neurodegenerative syndrome, ataxia-telangiectasia (A-T). This disease is characterized by pronounced cerebellar ataxia attributed to Purkinje cell (PC) degeneration, ocular telangiectasia, lymphoma, sterility, immunodeficiency, dysarthria and radiosensitivity (Chun \& Gatti, 2004; McKinnon, 2004). A-T results from null mutations in the gene ATM (ataxia-telangiectasia, mutated) (Savitsky et al., 1995). ATM is a serine/threonine kinase that modulates many substrates critical to the DNA damage response (Bakkenist \& Kastan, 2004; Shiloh, 2003; Shiloh, 2006). Tissue specificity is an important aspect of ATM function, and relates to the pleiotropic nature of A-T. For instance, some ATM deficient tissues are extremely radiosensitive such as the intestine or hematopoietic system, while in contrast ATM-deficient immature neurons are radioresistant (Frappart \& McKinnon, 2006).

Activation of ATM in response to DNA damage involves autophosphorylation, which converts ATM from an inactive dimer to an active monomer (Bakkenist \& Kastan, 2003). ATM autophosphorylation sites have been identified on serines 1981, 1893 and 367. Transfection of A-T cells with cDNAs containing mutations of these sites results in aberrant kinetics of p53 and Chk2 phosphorylation, suggesting each site contributes to ATM activity (Bakkenist \& Kastan, 2003; Kozlov et al., 2006). However, the in vivo significance of such events remains unclear. Transgenic mice expressing an S1987A mutated BAC were crossed onto an $\mathrm{Atm}^{-/-}$background and found to be indistinguishable from $\mathrm{Atm}^{+/}$mice, as growth retardation, genomic instability, lymphocyte development, and ATM-dependent phosphorylation of Chk2, SMC1, and p53 were restored in $\mathrm{Atm}^{\mathrm{TgS1} 987 \mathrm{~A}} \mathrm{Atm}^{-/-}$mice (Pellegrini et al., 2006). A transgenic mouse model in which all three currently known autophosphorylation sites were inactivated was recently reported. Atm-dependent DNA damage responses were normal in these mice. Thus, while phosphorylation of these sites is indicative of ATM activation, they do not appear to be required for ATM signaling itself(Daniel et al., 2008). 
An important issue is precisely how DNA DSBs signal to ATM. This occurs via the MRN complex. MRN-deficient cells show abrogated ATM autophosphorylation and impaired phosphorylation of $\mathrm{p} 53$, Chk2, and Smc1 concomitant with suppression of the ATM-dependent G2/M checkpoint (Carson et al., 2003; Difilippantonio et al., 2005; Uziel et al., 2003). These studies implicate the MRN complex as the primary sensor of DNA damage and place it upstream of ATM activation. Mechanistically, MRNdependent ATM activation at DNA breaks has been reported to require interaction between ATM HEAT repeats and the C-terminal FXF/Y domain of Nbs1 (Falck et al., 2005; You et al., 2005). The in vivo significance of this requires further study as while the NBS1 C-terminal mutant mice show apoptotic defects in the thymus, ATM functions were at least partially intact suggesting that interaction with the NBS1 C-terminus is dispensable for most ATM activity (Difilippantonio et al., 2007; Stracker et al., 2007).

In response to DNA damage, ATM phosphorylates p53 on serine 15 and other sites thereby directly mediating its activation and stabilization (Lavin \& Kozlov, 2007; Lukas et al., 2004). ATM-dependent phosphorylation activates Chk2, which blocks DNA replication via enhanced p53 activation and Cdc25A degradation (Falck et al., 2002). ATM indirectly affects p53 stabilization through down-regulation of its ubiquitin ligase Mdm2 (Khosravi et al., 1999; Maya et al., 2001). COP1 is yet another E3 ligase of p53 that ATM phosphorylates, resulting in its degradation and subsequent p53 stabilization (Dornan et al., 2006). These ATM-dependent modifications collectively function to affect p53-dependent cell cycle arrest or apoptosis.

In addition to directly regulating cell cycle checkpoints, apoptosis, and DNA repair, ATM has also been implicated in mediating DNA damage-induced chromatin modification. Treatment of primary human fibroblasts with chromatin modifying agents (chloroquine or HDAC inhibitors) induced ATM activation without forming obvious DNA DSBs, suggesting that direct DNA damage is not the only activator of ATM signaling (Bakkenist \& Kastan, 2003). Identification of ATM-dependent, DNA damageinduced chromatin relaxation via KAP-1 activity has further demonstrated the dependence of DNA repair on alteration of chromatin structure. ATM phosphorylates KAP-1 on Ser 824, and ablation of this site was demonstrated to abolish DSB-induced chromatin relaxation and resulted in cellular hypersensitivity to DSB-inducing agents (Ziv et al., 2006). Moreover, ATM has also been implicated in regulating ordered chromatin changes after DNA breaks to facilitate DNA repair (Berkovich et al., 2007). In essence ATM targets a vast array of substrates, which likely account for the pleiotropic nature of $\mathrm{A}-\mathrm{T}$.

\subsubsection{Ataxia-telangiectasia and Rad3-related (ATR)}

Mutations in the other major DNA repair kinase, ataxia-telangiectasia and $\operatorname{Rad} 3$ related (ATR), have been attributed to the microcephalic condition Seckel syndrome. While ATM responds to DSBs (such as induced by IR) through Chk2 activation, ATR targets Chk1 in response to DNA replication stress, and activates the intra-S phase checkpoint (Conti et al., 2007). Seckel syndrome is inherited autosomal recessively and 
characterized by microcephaly, intrauterine growth defects, dwarfism, and mild mental retardation (OMIM \#210600). In some affected individuals, hypomorphic splicing mutations in ATR have been identified as the disease causation (O'Driscoll et al., 2004; O'Driscoll et al., 2003a; O'Driscoll et al., 2003b). While there is reported cross-talk between ATM and ATR (Jazayeri et al., 2006; Matsuoka et al., 2007; Yoo et al., 2007), they have separable biochemical roles and complete ATR loss is incompatible with viability. Atr ${ }^{--}$embryos die between the blastocyst stage and E7.5. Isolation of $\mathrm{Atr}^{-{ }^{-}}$ embryos at day 3.5 revealed that blastocysts hatched normally from the zona pellucida and implanted onto tissue culture plastic after 1 day in culture, but failed to expand beyond 2 days in culture and died by culture day 4 due to accumulation of caspase 3independent DNA fragmentation and consequent apoptosis (Brown \& Baltimore, 2000). Despite structural similarity and overlapping substrates between ATR and ATM, there is unlikely to be significant redundancy in their roles during neurogenesis. Other microcephalic diseases associated with DSBR deficiency include LIG4 syndrome, Nijmegen breakage syndrome, and possibly primary microcephaly due to the recently described microcephalin gene $(\mathrm{MCPH})$.

\subsubsection{DNA ligase IV (Lig4)}

Human deficiency in DNA ligase IV causes microcephaly, developmental delay, radiosensitivity and immunodeficiency (O'Driscoll et al., 2001). LIG4 syndrome was first identified in a radiosensitive leukemia patient carrying a missense mutation (R278H) that conferred decreased ligation capacity (Riballo et al., 1999). This mutation was subsequently shown to decrease LIG4 adenylation and ligation activity by about $10 \%$ (O'Driscoll et al., 2001; Riballo et al., 1999; Riballo et al., 2001). Additional LIG4 syndrome individuals have since been identified with variable phenotypic penetrance dependant upon the particular hypomorphic mutations expressed. For instance, an individual with an $\mathrm{R} 278 \mathrm{H}$ mutation in addition to the N-terminal polymorphisms $\mathrm{A} 3 \mathrm{~V}$ and T9I presented with microcephaly, pancytopenia, and developmental delay (Girard et al., 2004; O'Driscoll et al., 2001). In vitro studies revealed no residual LIG4 adenylation or ligation activity in the 411BR mutation (Girard et al., 2004). Two siblings who presented with SCID, growth defects, and microcephaly were found to have compound heterozygous mutations conferring a Q280R substitution on one allele and a K424 frameshift mutation, resulting in truncation of the C-terminus on the other allele (Buck et al., 2006b). Given the lethality of mice with inactivated Lig4 the hypomorphic mutations present in the human syndrome suggests that loss of LIG4 would not be compatible with viability, and that even attenuated activity has a substantial developmental effect.

\subsubsection{The Mre11/Rad50/Nbs1 complex (MRN)}

While no human deficiency has been reported for Rad50, hypomorphic mutations in Mre11 and Nbs1 cause ataxia-telangiectasia like disease (ATLD) and Nijmegen breakage syndrome (NBS), respectively (Stewart et al., 1999; Varon et al., 1998). ATLD presents with cerebellar ataxia similar that seen in A-T, but less severe and with a longer 
latency, whereas NBS is characterized by microcephaly without neurodegeneration. Overlapping features of ATLD, A-T, and NBS include immunodeficiency, chromosomal instability, and reproductive defects (Pitts et al., 2001; Stewart et al., 1999; Varon et al., 1998). Given the differing neurologic phenotypes of A-T, ATLD, and NBS, despite overlapping features outside the brain, it is reasonable to suspect that there is tissuespecificity to Nbs1 function. Figure 1.1 summarizes the relationships between A-T, ATLD, NBS, ATR-SS, and LIG4 syndrome.

\subsubsection{Mouse models of DNA repair deficiencies}

Atm null mice have provided much insight into the nature of A-T by recapitulating many features of the disease, including growth retardation, immunodeficiency, sterility, and thymic lymphoma. However, no overt neurodegeneration has been observed in $\mathrm{Atm}^{-/-}$mice, thus impeding comprehension of how and why cerebellar degeneration occurs in A-T individuals (Barlow et al., 1996a; Elson et al., 1996b; Herzog et al., 1998; Xu et al., 1996a). Human A-T derived cell lines and mouse Atm-null cells display similar phenotypes including premature senescence, radiation sensitivity, decreased p53 induction and cell cycle checkpoint defects (Barlow et al., 1996a; Xu \& Baltimore, 1996; Xu et al., 1998).

Irradiation studies in the developing Atm-null NS showed that ATM regulates p53-dependent apoptosis of newly post-mitotic neurons, implying that it functions as a clearance mechanism of irreparably damaged cells during development. Post-mitotic neurons of the developing CNS and the dorsal root ganglia of postnatal $\mathrm{Atm}^{-/}$mice were resistant to irradiation-induced apoptosis in comparison to wild-type animals. Consistent with the lack of apoptosis, p53 stabilization was drastically attenuated in the radioresistant $\mathrm{Atm}^{-/-}$tissues and there was a similar lack of IR-induced neural apoptosis in p53-null animals (Chong et al., 2000; Herzog et al., 1998; Lee et al., 2001). Chk2, Puma, and Bax deficiencies have also been shown to abrogate IR-induced apoptosis in the developing nervous system (Chong et al., 2000; Jeffers et al., 2003; Takai et al., 2002).

The Mre $11^{A T L D 1 / A T L D 1}$ mouse model harboring a hypomorphic mutation designed to mimic mutations found in ATLD patients showed chromosomal instability and checkpoint defects in primary cell cultures, and Mre $11^{A T L D I / A T L D 1}$ females failed to produce offspring due to reduced embryonic viability (Theunissen et al., 2003). Likewise, the nibrin hypomorph $N b s 1^{\Delta B / \Delta B}$ mouse model did not recapitulate microcephaly as seen in NBS individuals, but did show a cellular phenotype identical to that of primary cultures derived from NBS patients including intra-S phase checkpoint defects, chromosomal instability, and radiosensitivity (Williams et al., 2002). Additionally, the $N B S 1^{m / m}$ mouse model displayed lymphoma predisposition and infertility, as did the humanized mouse model $h N b s 1^{657 \Delta 5}$ (Difilippantonio et al., 2005; Kang et al., 2002). Further understanding of NBS neuropathology came with the conditional Nbs1 knockout model, $N b n^{f 6 / f 6}$. On a Nestin-cre background, this mouse model demonstrated microcephaly and severe developmental defects of the cerebellum, associated with decreased proliferation of granule cell progenitors and increased apoptosis of inner 

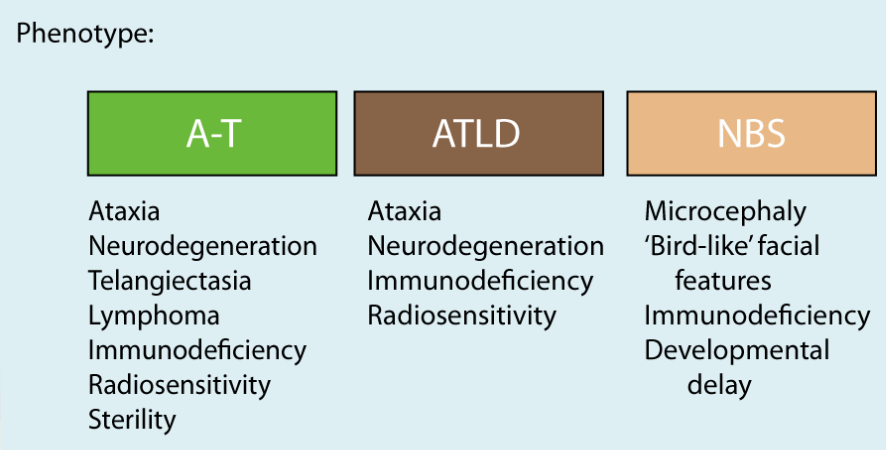

\section{ATR-Seckel LIG4 Syndrome}
Microcephaly Microcephaly
'Bird-like' facial Characteristic facial features features
Immunodeficiency Immunodeficiency
Developmental Developmental
delay delay

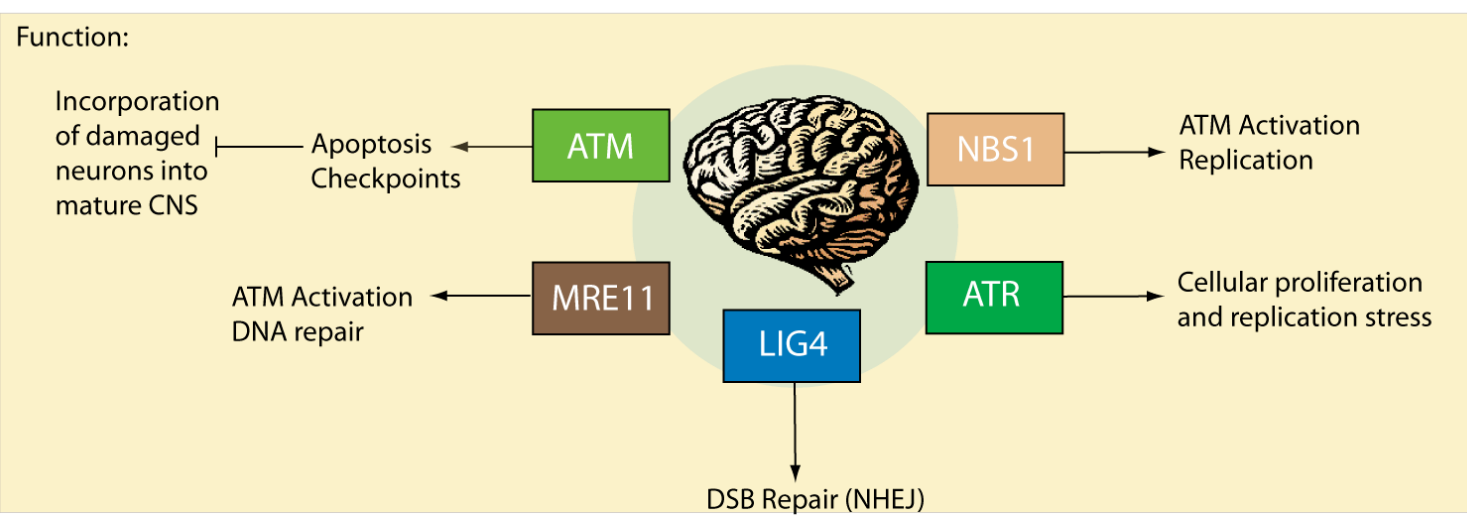

Figure 1.1 Diseases associated with DNA double-strand break repair deficits. Ataxia-telangiectasia mutated (ATM) deficiency results in a pleiotropic phenotype, the most debilitating feature of which is progressive neurodegeneration. MRE11 mutations are associated with A-T-like disease (ATLD), which mimics the neurodegenerative aspect of A-T. Though NBS1 acts with MRE11 to regulate ATM activation, NBS1 hypomorphic mutations are associated with microcephaly, not neurodegeneration. The Nijmegen breakage syndrome (NBS) phenotype overlaps with ATM and rad3-related (ATR) deficiency more so than with A-T. Mutation of LIG4 is also associated with microcephaly and resembles NBS and ATR-Seckel syndrome. 
external granule layer (EGL) cell populations (Frappart et al., 2005). While the $N b n^{f 6 / f 6}$ model provides insight into how DNA repair deficiency can affect the developing nervous system, the reason for reliance of the mature CNS upon ATM function remains obscure. Rescue of Lig4-mediated apoptosis by Atm-deficiency demonstrated the requirement for ATM-dependent apoptosis during neural development to maintain genomic integrity of cells incorporated into the mature nervous system (Lee et al., 2000; Sekiguchi et al., 2001). Accordingly, p53 deficiency rescued Xrcc4/Lig4-mediated apoptosis as well (Frank et al., 2000). Interestingly, Lig $4^{-/} p 53^{-/-}$mice survived into adulthood and developed pro-B cell lymphomas or medulloblastomas (Frank et al., 2000; Lee \& McKinnon, 2002), whereas $\mathrm{Lig}^{-/-} \mathrm{Atm}^{-/}$animals died perinatally (Lee et al., 2000; Sekiguchi et al., 2001). These studies suggest that ATM deficiency allows cells carrying a genotoxic level of DSBs to be incorporated into the nervous system, and eventually such neurons would succumb to apoptosis or some other form of death resulting in neurodegeneration (Lee \& McKinnon, 2007). Furthermore, these studies indicate a developmental role of ATM in addition to mediation of p53-dependent apoptosis that are vital in responding to DNA damage. Table 1.1 summarizes relevant mouse models and the degree to which their respective diseases are phenocopied.

The DNA DSB response is required for development and maintenance of tissue homeostasis. The DNA damage response is also a barrier to transformation, and if inactive in the face of replication stress, cellular transformation can occur (Bartkova et al., 2005; Bartkova et al., 2006; Di Micco et al., 2006). Multiple human syndromes result from the mutation of genes required for the response to DNA DSBs. In many cases these syndromes feature defective developmental processes, including microcephaly, neurodegeneration, immunodeficiency, and growth retardation. Delineating the underlying molecular defects in these diseases promises to uncover further important details about DNA DSB repair (Phillips \& McKinnon, 2007).

The molecular mechanisms responsible for the distinct neuropathologies observed in ATLD and NBS are of particular interest, as Mre11 and Nbs1 are both implicated in ATM activation, coordination of the DSB response, and maintaining the integrity of cellular proliferation during early mammalian development. The fact that hypomorphic mutations in Mre11 cause neurodegeneration while Nbs1 mutations have been associated with microcephaly suggests that different downstream signaling events occur in ATLD and NBS. In Chapter 2 of this work, mouse models for ATLD and NBS were used to evaluate the consequences of hypomorphic mutations in either Mre11 or Nbs1 in the nervous system, as pertaining to DSB signaling. Similar to NBS, ATR-Seckel syndrome is associated with developmental delay and microcephaly. ATM and ATR are very similar kinases that mediate the DNA DSB response, but human deficits in these proteins result in very different consequences. To better understand the relationship between ATM and ATR signaling in the nervous system, a conditional Atr knock-out mouse model was used to specifically evaluate how Atr functions in the nervous system to ensure developmental integrity. Double conditional Atr, Atm mutant mice were generated to assess how Atm and Atr cooperate in development and maintenance of the CNS. The work presented in this dissertation has provided valuable new insights into the signaling pathways that prevent neuropathology associated with DNA damage. 
Table 1.1 Phenotypes resulting from inactivation of murine DNA damage response genes.

\begin{tabular}{|c|c|c|c|c|}
\hline Gene & Mouse Model & Mouse Phenotype & $\begin{array}{l}\text { Human } \\
\text { Disease }\end{array}$ & Human Phenotype \\
\hline$\overline{A T M}$ & $\begin{array}{l}\mathrm{Atm}^{-/-} \\
\text {(Barlow et al., } \\
\text { 1996b; Elson et } \\
\text { al., 1996a; Xu et } \\
\text { al., 1996b) }\end{array}$ & $\begin{array}{l}\text { Thymoma } \\
\text { predisposition, } \\
\text { sterility, } \\
\text { radiosensitivity }\end{array}$ & $\begin{array}{l}\text { Ataxia- } \\
\text { telangiectasia }\end{array}$ & $\begin{array}{l}\text { Cerebellar ataxia, } \\
\text { ocular telangiectasia, } \\
\text { thymoma, sterility, } \\
\text { genomic instability, } \\
\text { radiosensitivity }\end{array}$ \\
\hline \multirow[t]{5}{*}{$N B S$} & $\begin{array}{l}N B S 1^{m / m} \\
\text { (Kang et al., } \\
2002 \text { ) }\end{array}$ & $\begin{array}{l}\text { Thymoma } \\
\text { predisposition, } \\
\text { sterility, } \\
\text { radiosensitivity }\end{array}$ & \multirow[t]{5}{*}{$\begin{array}{l}\text { Nijmegen } \\
\text { breakage } \\
\text { syndrome }\end{array}$} & \multirow[t]{5}{*}{$\begin{array}{l}\text { Microcephaly, } \\
\text { growth retardation, } \\
\text { immunodeficiency, } \\
\text { characteristic facial } \\
\text { features, lymphoma }\end{array}$} \\
\hline & $\begin{array}{l}\mathrm{Nbn}^{f 6 / f 6} \text {; nestin- } \\
\mathrm{Cre} \\
\text { (Frappart et al., } \\
\text { 2005) }\end{array}$ & $\begin{array}{l}\text { Microcephaly, } \\
\text { ataxia, cerebellar } \\
\text { development defects }\end{array}$ & & \\
\hline & $\begin{array}{l}N B S 1^{-/-} \\
\text {(Zhu et al., 2001) }\end{array}$ & $\begin{array}{l}\text { Embryonic lethality } \\
(\sim \mathrm{E} 7.5)\end{array}$ & & \\
\hline & $\begin{array}{l}N B S 1^{\Delta B / \triangle B} \\
\text { (Williams et al., } \\
\text { 2002) }\end{array}$ & $\begin{array}{l}\text { Cell cycle } \\
\text { checkpoint defect in } \\
\text { MEFs, synthetic } \\
\text { lethal with } \mathrm{Atm}^{-/-}\end{array}$ & & \\
\hline & $\begin{array}{l}N N b s 1^{657 \Delta 5} \\
\text { (Difilippantonio } \\
\text { et al., 2005) }\end{array}$ & $\begin{array}{l}\text { Impaired } \\
\text { development, } \\
\text { lymphoma }\end{array}$ & & \\
\hline MRE11 & $\begin{array}{l}\text { Mre } 11^{\text {ATLDI/ATLDI }} \\
\text { (Theunissen et al., } \\
\text { 2003) }\end{array}$ & $\begin{array}{l}\text { Cell cycle } \\
\text { checkpoint defect in } \\
\text { MEFs, synthetic } \\
\text { lethal with } \mathrm{Atm}^{-/-}\end{array}$ & $\begin{array}{l}\text { Ataxia- } \\
\text { telangiectasia } \\
\text { like disease }\end{array}$ & $\begin{array}{l}\text { Cerebellar ataxia, } \\
\text { genomic instability }\end{array}$ \\
\hline $\begin{array}{l}\text { DNA } \\
\text { Ligase IV }\end{array}$ & $\begin{array}{l}\text { Lig4 }^{-/-} \\
\text {(Barnes et al., } \\
\text { 1998; Frank et al., } \\
\text { 1998) }\end{array}$ & $\begin{array}{l}\text { Embryonic lethality } \\
(\sim \text { E16.5), neuraxis } \\
\text { wide apoptosis, } \\
\text { immunodeficiency }\end{array}$ & $\begin{array}{l}\text { LIG4 } \\
\text { syndrome }\end{array}$ & $\begin{array}{l}\text { Microcephaly, } \\
\text { characteristic facial } \\
\text { features, } \\
\text { immunodeficiency, } \\
\text { radiosensitivity, } \\
\text { growth retardation }\end{array}$ \\
\hline \multirow[t]{2}{*}{$A T R$} & $\begin{array}{l}\text { Lig4 }{ }^{Y 288 C} \\
\text { hypomorph } \\
\text { (Nijnik et al., } \\
\text { 2007) }\end{array}$ & $\begin{array}{l}\text { Growth retardation, } \\
\text { immunodeficiency, } \\
\text { hematopoietic stem } \\
\text { cell deficiency }\end{array}$ & \multirow[t]{2}{*}{$\begin{array}{l}\text { Seckel } \\
\text { syndrome }\end{array}$} & \multirow{2}{*}{$\begin{array}{l}\text { Microcephaly, } \\
\text { characteristic facial } \\
\text { features, } \\
\text { chromosomal } \\
\text { instability }\end{array}$} \\
\hline & $\begin{array}{l}\text { Atr }^{-/-} \\
\text {(Brown \& } \\
\text { Baltimore, 2000) }\end{array}$ & $\begin{array}{l}\text { Embryonic lethality } \\
\text { (before E7.5) }\end{array}$ & & \\
\hline
\end{tabular}




\section{CHAPTER 2 DIFFERENTIAL DNA DAMAGE SIGNALING ACCOUNTS FOR DISTINCT NEUROPATHOLOGY ASSOCIATED WITH ATLD AND NBS*}

\subsection{Introduction}

The developing nervous system is highly susceptible to DNA damage, and many human syndromes caused by DNA repair deficiency present with pronounced neuropathology (Katyal \& McKinnon, 2007; McKinnon \& Caldecott, 2007; Rass et al., 2007). Within the developing nervous system, DNA DSBs pose a significant threat and are normally repaired by either non-homologous end joining (NHEJ) or homologous recombination (HR) (Lees-Miller \& Meek, 2003; Lieber et al., 2003; West, 2003). Coincident with repair, DNA DSBs also activate cell cycle checkpoints to pause cellular proliferation and ensure fidelity of repair and the maintenance of genomic integrity (Kastan \& Bartek, 2004; Shiloh, 2006; Zhao et al., 2000). An alternative outcome from DNA DSBs, and one that is frequent during neural development is the activation of apoptosis to eliminate DNA-damaged cells (Difilippantonio et al., 2007).

Mre11/Rad50/NBS1 (MRN) and ATM (ataxia telangiectasia, mutated) are required to coordinate the DNA DSB signaling response (Kastan \& Bartek, 2004; Lavin \& Kozlov, 2007; Petrini \& Stracker, 2003; Shiloh, 2006). The MRN complex rapidly localizes to DNA DSBs, where it recruits and activates the ATM kinase to initiate DNA damage signaling (Carson et al., 2003; Lee \& Paull, 2004; Uziel et al., 2003). ATM initiates a signaling cascade via phosphorylation of multiple substrates resulting in cell cycle checkpoints or apoptosis (Uziel et al., 2003). In the nervous system p53 and Chk2 are particularly important (Lee et al., 2001; Takai et al., 2002). MRN modulation of ATM activation requires protein-protein interactions with the NBS1 C-terminus (Falck et al., 2005), although the ATM-MRN interplay is complex and likely to involve multiple protein interactions in determining the outcome of DNA DSB signaling (Difilippantonio et al., 2007; Stracker et al., 2007).

Underscoring the importance of the DSB response is the occurrence of diseases when mutations render ATM, Mre11 or NBS-1 dysfunctional and result in ataxia telangiectasia (A-T), A-T-like disease (ATLD) or Nijmegen breakage syndrome (NBS) respectively (Kobayashi et al., 2004; McKinnon, 2004; Taylor et al., 2004). Unlike ATM, MRE11 and NBS1 are essential during development (Xiao \& Weaver, 1997; Zhu et al., 2001), thus syndromes involving these genes result from hypomorphic mutations. A-T is primarily characterized by cerebellar ataxia associated with Purkinje cell degeneration

*Adapted with permission from Shull ERP, Lee Y, Nakane H, Stracker TH, Zhao J, Russell HR, Petrini JHJ, and McKinnon PJ. (2009). Genes Dev, 23(2) 171-180. 
(McKinnon, 2004). Other phenotypic characteristics of A-T are immunodeficiency, radiosensitivity, ocular telangiectasia, sterility and cancer predisposition. ATLD is also characterized by neurodegeneration and radiosensitivity (Taylor et al., 2004). In contrast, NBS features microcephaly, immunodeficiency, radiosensitivity, and cancer predisposition (Kobayashi et al., 2004). The overlapping phenotypes of A-T, ATLD, and NBS presumably reflect important functional interactions between ATM and the MRN complex. However, the distinct neuropathology present in ATLD compared to NBS indicates that the hypomorphic MRN mutations that give rise to these diseases must ultimately lead to different downstream signaling events to account for the distinct neuropathology. How this occurs is unknown.

Using mouse models for ATLD and NBS, an in vivo analysis of the functional consequence of these hypomorphic mutations in the nervous system was performed. Exogenous and endogenous genotoxic stress was induced to evaluate the relative roles of ATM, Mre11 and Nbs1 during neural development and to investigate the signaling defects that could account for the distinct neuropathology characteristic of each disease. Our data show that the disease-causing mutations in MRN lead to defects in DNA damage-induced signal transduction in the nervous system, whereby the relative activation of apoptosis may underpin the differential neuropathology of these syndromes.

\subsection{Experimental Procedures}

\subsubsection{Animals}

To generate a conditional DNA Ligase IV allele, Dr. Helen Russell obtained a murine BAC genomic clone (strain, 129Ola) containing an $\sim 13 \mathrm{~kb}$ BamHI fragment with the Lig4 genomic locus, into which she placed a LoxP sequence into a unique MluI site $4.8 \mathrm{~kb}$ upstream of the Lig4 ORF and a floxed NeoTK construct cloned as a HindIII fragment $\sim 1.7 \mathrm{~kb}$ downstream to generate $\mathrm{pLig} 4^{\mathrm{LoxP}}$. ES cells (W9.5; 129S1/SvImJ) were electroporated with linearized $\mathrm{pLig} 4^{\mathrm{LoxP}}$ and selected with G418 antibiotic and correctly targeted cells were identified by Southern Blot analysis. Targeted ES clones were then electroporated with pMC-cre, followed by selection in FIAU (5-iodo-2'-fluoro-2'deoxy-1arabinofuranosyluracil) to kill cells containing TK. ES cells were then injected into blastocysts and the resultant chimeric mice assessed for germline transmission of the floxed Lig4 allele (St. Jude Children's Research Hospital Transgenic Core). Nestin-cre mice (B6.Cg-Tg(Nes-cre)1Kln/J; JAX \#003771) were used to delete Lig4 throughout the nervous system.

Animals were housed in an Association for the Assessment and Accreditation of Laboratory Animal Care (AAALAC)-accredited facility and were maintained in accordance with the NIH Guide for the Care and Use of Laboratory Animals. The St. Jude Children's Research Hospital IACUC approved all procedures for animal use. 


\subsubsection{Histology and immunohistochemistry}

Mice were sacrificed via transcardial perfusion using 4\% paraformaldehyde (PFA). Tissues were further fixed in 4\% PFA overnight, and either cryoprotected in $25 \%$ PBS-buffered sucrose solution and embedded in O.C.T. (Tissue-Tek) or paraffinembedded. Frozen tissue was sectioned at $10 \mu \mathrm{m}$ thickness using a Microm HM500 M cryostat. Paraffin-embedded spinal cord samples were sectioned at $40 \mu \mathrm{m}$ thickness using a Microm HM 325.

Antigen retrieval was used for all immunohistochemistry using the anti-phospho$\gamma \mathrm{H} 2 \mathrm{AX}$ (1:500; Abcam), anti-53BP1 polyclonal (1:500; Bethyl laboratories), anti-p53 (CM5; 1:500; Vector Laboratories); anti-p53 phospho-Ser-15 (1:500; Cell Signaling), and anti-active caspase 3 (1:500; BD Biosciences) antibodies. For immunofluorescent staining, sections were subsequently immunolabelled with Cy3-conjugated goat antimouse (1:400; Jackson Immunoresearch) and/or FITC-conjugated goat anti-rabbit secondary antibodies (1:500; Jackson Immunoresearch) and mounted with Vectashield anti-fade reagent containing DAPI (Vectorlabs). Immunohistochemistry for activated caspase-3 or p53-ser 15 was performed overnight at room temperature after quenching endogenous peroxidase activity with $0.6 \%$ hydrogen peroxide in methanol.

Immunoreactivity was visualized with a vasoactive intestinal peptide substrate kit (V.I.P.; Vector Labs) according to the manufacturer's directions after tissues were treated with biotinylated secondary antibody and avidin DH-biotinylated horseradish peroxidase- $\mathrm{H}$ complex (Vectastain Elite kit; Vector Labs). Sections were counterstained with $0.1 \%$ methyl green (Vector Labs), dehydrated, and mounted in DPX reagent. Apoptotic cells were detected with ApopTag Fluorescein in situ apoptosis detection kit (Chemicon international) according to the manufacturer's instructions. $\gamma \mathrm{H} 2 \mathrm{AX}$ and 53BP1 foci were quantitated manually under the microscope at 400x magnification. Quantitation of TUNEL or caspase-3 positive cells was determined by counting positive signal from images equivalent in size and magnification between genotypes. Images were captured using an Axioskop 2.0 microscope (Carl Zeiss) and a SPOT camera (Diagnostic Instruments, Inc).

\subsubsection{Astrocyte culture}

Cerebral cortices were isolated from $\mathrm{P} 4$ mice and placed into a $35 \mathrm{~mm}$ dish containing ice cold 1:1 mix of Dulbecco's modified Eagle's medium and Ham's nutrient mixture F-12 (DMEM-F12; Gibco) supplemented with $2 \mathrm{mM} \mathrm{L}$-glutamine, $100 \mathrm{U} / \mathrm{mL}$ penicillin, and $100 \mu \mathrm{g} / \mathrm{mL}$ streptomycin. Meninges were removed under a dissecting microscope and cortices were transferred to a new dish containing fresh media mix as described above. Cortices were dissociated by passage through a $5 \mathrm{~mL}$ pipette, then centrifuged at $1000 \mathrm{rpm}$ for $5 \mathrm{~min}$. Cells were resuspended in DMEM-F12 supplemented with $10 \%$ fetal calf serum, $2 \mathrm{mM} L$-glutamine, $100 \mathrm{U} / \mathrm{mL}$ penicillin, $100 \mu \mathrm{g} / \mathrm{mL}$ streptomycin, and $20 \mathrm{\eta g} / \mathrm{mL}$ epidermal growth factor (Sigma). Astrocytes were allowed to establish in Primeria T-25 tissue culture flasks (Falcon) at $37^{\circ} \mathrm{C}$ in a humidified incubator. Medium was refreshed after 4 days in culture and cells were trypsinized at 
$100 \%$ confluence (typically $\sim 1$ week post-isolation). Cells were seeded onto 4-chambered poly-D-lysine coated slides (Falcon) at $2 \times 10^{4}$ cells/chamber and allowed to establish for 2 days prior to treatment and staining.

Cells were irradiated using a Gammacell® 40 Exactor (MDS Nordion). Following irradiation, slides were returned to incubator. After the designated recovery time, cells were washed in PBS for 5 min at RT, then fixed in 3\% PFA/ 0.2\% triton-X100 for 15 min at $4{ }^{\circ} \mathrm{C}$. Blocking solution (1.5\% milk in PBS) was applied for $1 \mathrm{hr}$ at RT, followed by incubation with anti-phospho- $\gamma \mathrm{H} 2 \mathrm{AX}\left(1: 1000\right.$; Abcam) overnight at $4{ }^{\circ} \mathrm{C}$. Primary antibody solution was aspirated from slide chambers, and cells were washed in PBS. Cells were subsequently immunolabelled with FITC-conjugated goat anti-rabbit secondary antibodies (1:500; Jackson Immunoresearch) and mounted with Vectashield anti-fade reagent containing DAPI (Vectorlabs). Images were captured at 400x magnification using an Axioskop 2.0 microscope (Carl Zeiss) and a SPOT camera (Diagnostic Instruments, Inc). Quantitation of foci was performed using the Adobe ${ }^{\circledR}$ Photoshop ${ }^{\circledR}$ plugin Image Processing Tool Kit (Reindeer Graphics, Inc.).

\subsubsection{Real-time PCR}

Genomic DNA was phenol/chloroform extracted from frozen sections and suspended in TE buffer. DNA concentration was determined from the $\mathrm{A}_{260}$ as measured using an Ultrospec 2000 UV/Visible Spectrophotometer (Pharmacia Biotech). Standard DNA was serially diluted at 1:5 from a starting concentration of $100 \mathrm{ng} / \mu \mathrm{L}$. Unknown samples were diluted to $100 \eta \mathrm{g} / \mu \mathrm{L}$, such that each reaction contained an equal amount of DNA. DNA was added to $\mathrm{iQ}^{\mathrm{TM}}$ SYBR ${ }^{\circledR}$ Green supermix (BioRad) containing forward and reverse primers for either the Lig4 coding region or $\beta$-actin. PCR reaction was carried out using the iQ ${ }^{\mathrm{TM}} 5$ Multicolor Real-Time PCR Detection System (BioRad). Measurement numbers were automatically assigned based on SYBR green amounts fit to a standard curve. The amount of Lig4 product was normalized to that of $\beta$-actin for each sample.

Lig4 forward primer: 5' ACTATACCGACCAGTTTGGTGAATC

Lig4 reverse primer: 5' CCCGAACGCATTGTGAATAA

ק-actin forward primer: 5' CAGCAAGCAGGAGTACGATGAG

$\boldsymbol{\beta}$-actin reverse primer: 5' CAGTAACAGTCCGCCTAGAAGCA

\subsection{Results}

\subsubsection{Kinetics of H2AX phosphorylation in Atm, Mre11, Nbs1, or Lig4-deficient astrocytes}

Histone $\mathrm{H} 2 \mathrm{~A}$ is conserved between eukaryotes and is classified into the subfamililies $\mathrm{H} 2 \mathrm{~A} 1, \mathrm{H} 2 \mathrm{~A} 2, \mathrm{H} 2 \mathrm{AZ}$, and $\mathrm{H} 2 \mathrm{AX}$. The $\mathrm{NH}_{2}$ - and $\mathrm{COOH}$ - tails are targets for 
post-translational modifications including phosphorylation, methylation, acetylation, and ubiquitination (Takahashi \& Ohnishi, 2005). Phosphorylation of H2AX serine 129 at the $-\mathrm{COOH}$ terminus is one of the first responses to DNA DSBs, occurring within 3 minutes following irradiation. This phosphorylation event has been attributed to overlapping activity between ATM and DNA-PKcs in non-replicating cells (Kuhne et al., 2004; Rogakou et al., 1998; Stiff et al., 2004). The modified histone is termed $\gamma$-H2AX, and can be visualized through immunoflourescence as punctate nuclear foci. Pulse-field gel electrophoresis (PFGE) is an established method of measuring DSBs that has been shown to correlate with the amount of $\gamma-\mathrm{H} 2 \mathrm{AX}$ foci observed in cells after irradiation, and is thus widely used as a quantitative measure of DSB repair. Both methods were employed to study repair defects in fibroblasts isolated from an A-T individual and a LIG4 syndrome patient. The A-T cells showed no deficient repair based on PFGE and prolonged persistence of $\gamma-\mathrm{H} 2 \mathrm{AX}$ foci after IR. In contrast, the LIG4 syndrome cells showed proficient repair even several days after prolonged exposure to IR (Kuhne et al., 2004).

Astrocytes were isolated from $\mathrm{Lig} 4^{\mathrm{Nes}-\mathrm{cre}}, \mathrm{Atm}^{-/}, \mathrm{Mrel}^{\mathrm{ATLDI/ATLD1}}$, and $\mathrm{Nbs} \mathrm{1}^{\triangle B / \triangle B}$ post-natal day $4(\mathrm{P} 4)$ pups to compare the DNA damage response in murine neural cells. In contrast to the Kuhne et al. report of proficient resolution of DSBs in LIG4 syndrome fibroblasts, $\operatorname{Lig} 4^{\text {Nes-cre }}$ astrocytes showed increased presence of $\gamma$-H2AX foci in untreated cells and dramatic persistence of foci over a prolonged recovery time. This difference from the LIG4 syndrome fibroblasts reflects the diminished repair capacity associated with LIG4 hypomorphic mutations, in contrast to complete loss of Lig4. Thus, Lig $4^{\text {Nes-cre }}$ astrocytes served as a positive control for DNA DSB repair deficiency. Concurrent with reported findings in A-T fibroblasts, Atm $^{-/-}$astrocytes showed abrogated $\mathrm{H} 2 \mathrm{AX}$ phosphorylation at earlier times post-IR, but there was a statistically insignificant increase in residual foci 6 hours post-IR perhaps suggesting a minor DSB repair deficiency. Because ATM is reportedly the major kinase responsible for H2AX phosphorylation (ATR and DNA-PK share H2AX as a common substrate as well), we reasoned that the Mre $11^{A T L D I / A T L D 1}$ and $N b s 1^{\triangle B / \triangle B}$ mutations would mimic repair kinetics in $\mathrm{Atm}^{-/}$astrocytes if Atm activity was compromised (Burma and Chen, 2004; Stiff et al., 2004; Wang et al., 2005b). Mre $11^{\text {ATLDI/ATLD1 }}$ astrocytes showed a pattern of H2AX phosphorylation that was largely indistinguishable from that seen in the $\mathrm{Atm}^{-/-}$cells. $N b s 1^{\Delta B / \triangle B}$ astrocytes showed a less pronounced abrogation of $\mathrm{H} 2 \mathrm{AX}$ phosphorylation 10 min post-IR than observed in either the $W T$ or Mre $11^{\text {ATLD1/ATLD1 }}$ cells. However, 6 hours post-IR, the $N b s 1^{\triangle B / A B}$ astrocytes showed a statistically insignificant increase in the number of residual foci compared to the $W T$ control (Figures 2.1 and 2.2). Variability and large standard deviations make this data difficult to interpret, thus firm conclusions cannot be drawn from it.

\subsubsection{IR-induced apoptosis is defective in the Mre11 $1^{A T L D 1 / A T L D 1}$ brain}

To determine the basis for the differential neuropathology associated with ATLD and NBS we used mice harboring hypomorphic alleles (Mre $11^{A T L D 1 / A T L D 1}$ and $\mathrm{Nbs} 1^{\triangle B / \triangle B}$ ) corresponding to the human diseases (Carney et al., 1998; Stewart et al., 1999; Varon et 


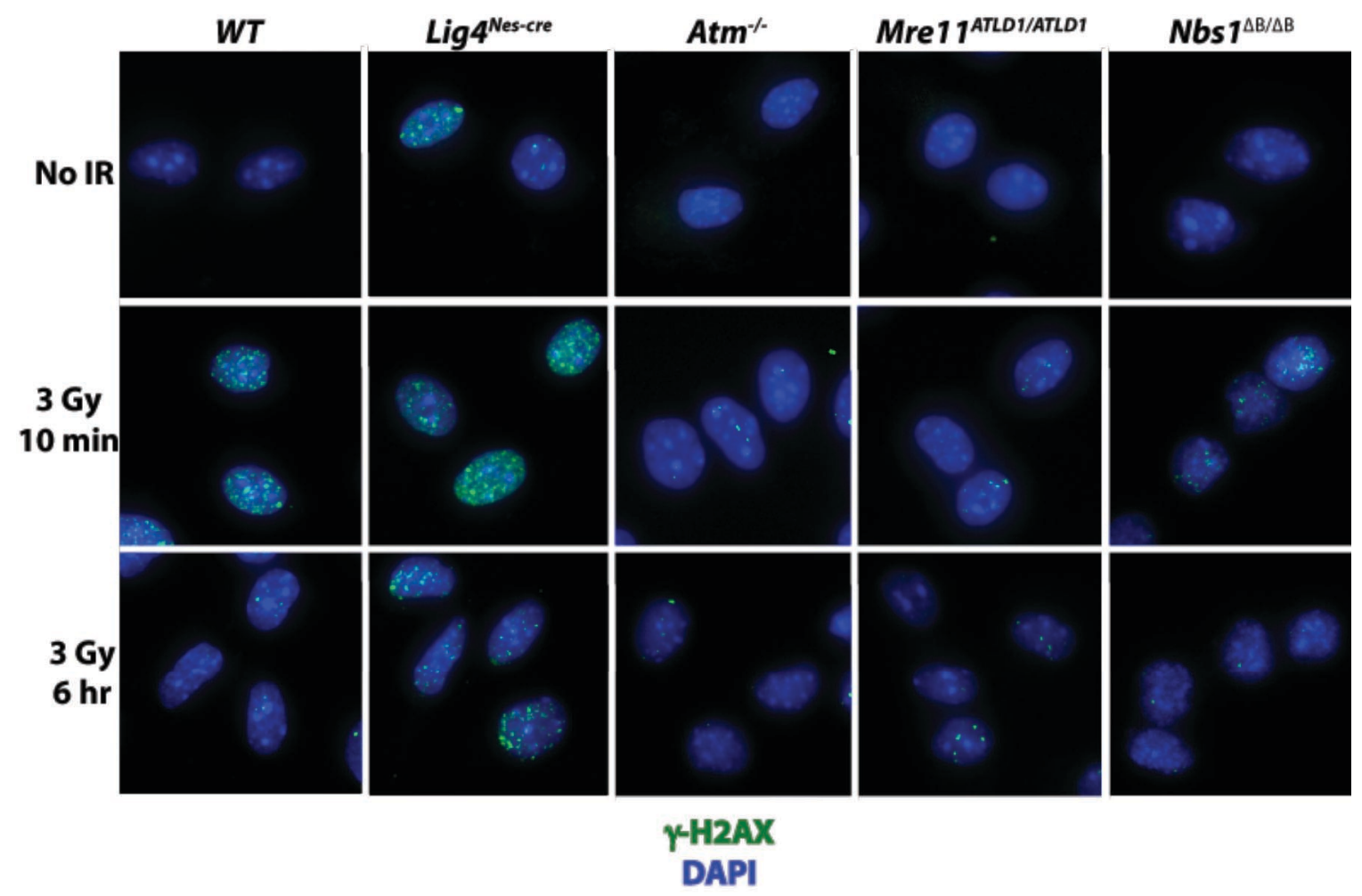

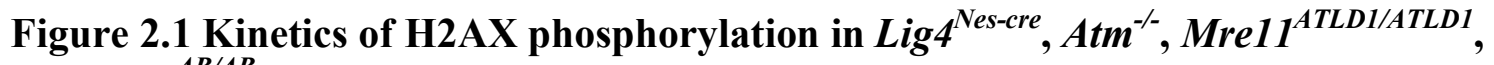
and $N b s 1^{\Delta B / A B}$ astrocytes.

Astrocytes were generated from P4 mice of the indicated genotypes. Cells were seeded onto chambered slides and allowed to grow 48 hours prior to being either mock treated or irradiated at a dose of 3 grays (Gy). Cells were allowed to recover for the indicated times, then were stained via indirect immunofluorescence against $\gamma$-H2AX (green). Nuclei were counterstained with DAPI (blue). Images were taken at 400x magnification. 


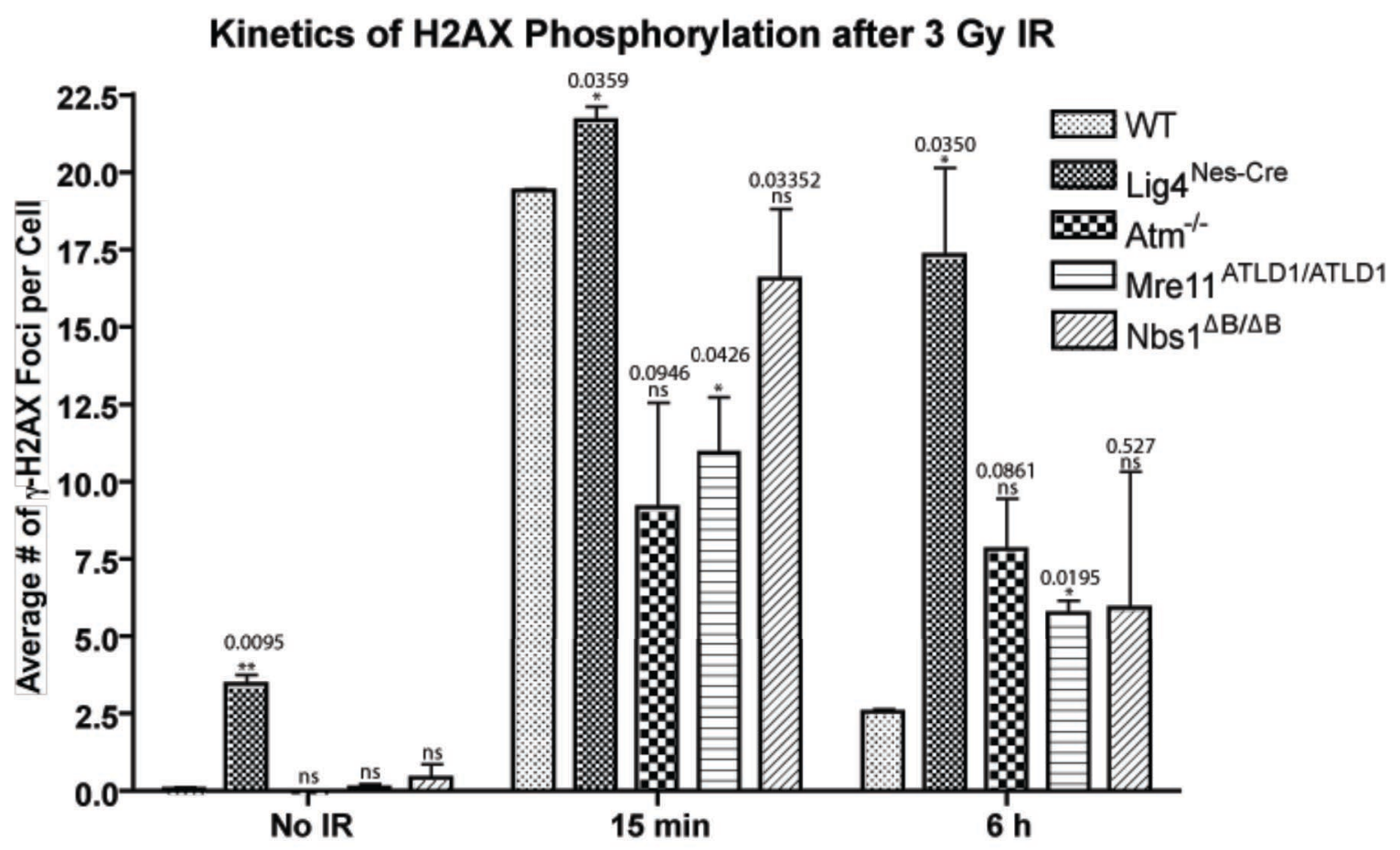

Figure 2.2 DNA DSB repair kinetics in Lig $4^{\text {Nes-cre }}, \mathrm{Atm}^{-/-}, \mathrm{Mre}^{\mathrm{ATLD1/ATLD1}}$, and $N b s 1^{A B / A B}$ astrocytes. $\gamma-\mathrm{H} 2 \mathrm{AX}$ foci were quantified from the images represented in Figure 2.1 using the Adobe ${ }^{\circledR}$ Photoshop ${ }^{\circledR}$ plugin Image Processing Tool Kit (Reindeer Graphics, Inc.). Error bars represent standard deviation between the results of 2 independent experiments performed with cells isolated from different animals. For each experiment, foci were counted in no less than 20 cells per genotype. 
al., 1998). The ATLD mutation $(633 \mathrm{R} \rightarrow \mathrm{STOP})$ results in a truncated Mre11 protein, while the NBS mouse has a truncating mutation of Nbs1 that produces an $80 \mathrm{kDa}$ protein lacking the N-terminal FHA and BRCT domains (Theunissen et al., 2003; Williams et al., 2002). These mice recapitulate many aspects of the respective human syndromes including radiosensitivity and cell cycle checkpoint defects, although they do not develop overt neuropathology (Theunissen et al., 2003; Williams et al., 2002).

While many features of NBS and ATLD can be attributed to a defect in ATM activation (Carson et al., 2003; Difilippantonio et al., 2005; Uziel et al., 2003), the disparate neuropathology suggests differences in DNA damage signaling in the nervous system. To investigate DNA damage responses in the developing nervous system of ATLD and NBS mice, we used ionizing radiation (IR) to induce genotoxic stress. Because apoptosis is a primary readout of IR-induced DNA damage in the developing nervous system (Difilippantonio et al., 2007), we analyzed this in the postnatal retina and dentate gyrus using immunohistochemistry to assess caspase-3 activation. In the absence of radiation no caspase-3 positive cells were seen (Figure 2.3). However after radiation, wild type (WT) and $N b s 1^{\triangle B / A B}$ mice showed an equivalent susceptibility to IR-induced apoptosis (Figure 2.3A c-f). In contrast, there was a marked resistance to apoptosis in the Mre $11^{A T L D 1 / A T L D 1}$ mice (Figure 2.3A g,h). IR-induced cell death was substantially reduced throughout immature postmitotic regions of the Mre $11^{\text {ATLDI/ATLD1 developing }}$ nervous system, including the inner nuclear layer of the retina and the dentate gyrus of the hippocampus (Figure 2.3A g, h). The resistance of these particular cell populations is similar to that observed in the same regions in the $\mathrm{Atm}^{-/-}$brain (Herzog et al., 1998; Lee et

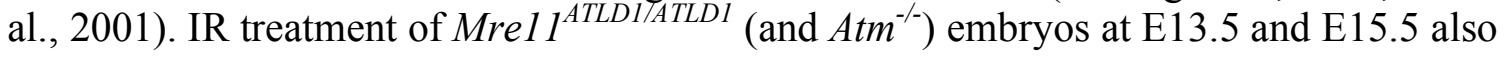
showed resistance to apoptosis in immature, postmitotic neurons akin to the P5 tissues, while in $N b s 1^{\Delta B / A B}$ embryos IR-induced apoptosis was indistinguishable from WT embryos. Importantly, IR-induced apoptosis in the proliferating ventricular zone of the embryonic nervous system occurred normally in Mre $11^{A T L D 1 / A T L D 1}$ (and $N b s 1^{\triangle B / \triangle B}$ ) as it does in $\mathrm{Atm}^{-/-}$tissue (data not shown). Thus, ATLD but not NBS mutations measurably influence DNA damage-induced apoptosis in specific regions of the developing brain.

Because the MRN complex can modulate ATM kinase activity, we determined if the resistance to IR-induced apoptosis in the Mre $11^{A T L D 1 / A T L D 1}$ tissue was related to defective Atm activity. Dr. Youngsoo Lee, in the McKinnon lab, assayed Atm ser1987 phosphorylation (equivalent to ser1981 of human ATM) as a marker for DNA damageinduced Atm activation in vivo in Mre $11^{A T L D 1 / A T L D 1}$ and $N b s 1^{\triangle B / \triangle B} \mathrm{P} 5$ cerebellum, a tissue significantly affected in both diseases. At doses of $1 \mathrm{~Gy}$ or below, very little Atm ser1987 phosphorylation was observed in either Mre $11^{A T L D 1 / A T L D 1}$ or $N b s 1^{\triangle B / \triangle B}$ tissue compared to WT cerebellum (Figure 2.4A). However, at higher radiation levels ( $>4 \mathrm{~Gy}$ ) Atm phosphorylation was evident in Mre $11^{A T L D 1 / A T L D 1}$ and $N b s 1^{\triangle B / \triangle B}$ tissue, albeit reduced compared to WT (Figure 2.4A). These data indicate that both Mre11 and Nbs1 mutations result in reduced Atm ser1987 phosphorylation, indicating MRN modulates Atm signaling in the developing brain. As $N b s 1^{\Delta B / \Delta B}$ neural tissue underwent IR-induced apoptosis similar to WT (Figure 2.3), defective Atm phosphorylation was somewhat unexpected. Therefore, we confirmed disruption of the MRN complex in Mre $11^{A T L D 1 / A T L D I}$ and $N b s 1^{\triangle B / \triangle B}$ tissue. Jingfeng Zhao, in the McKinnon lab, showed that 

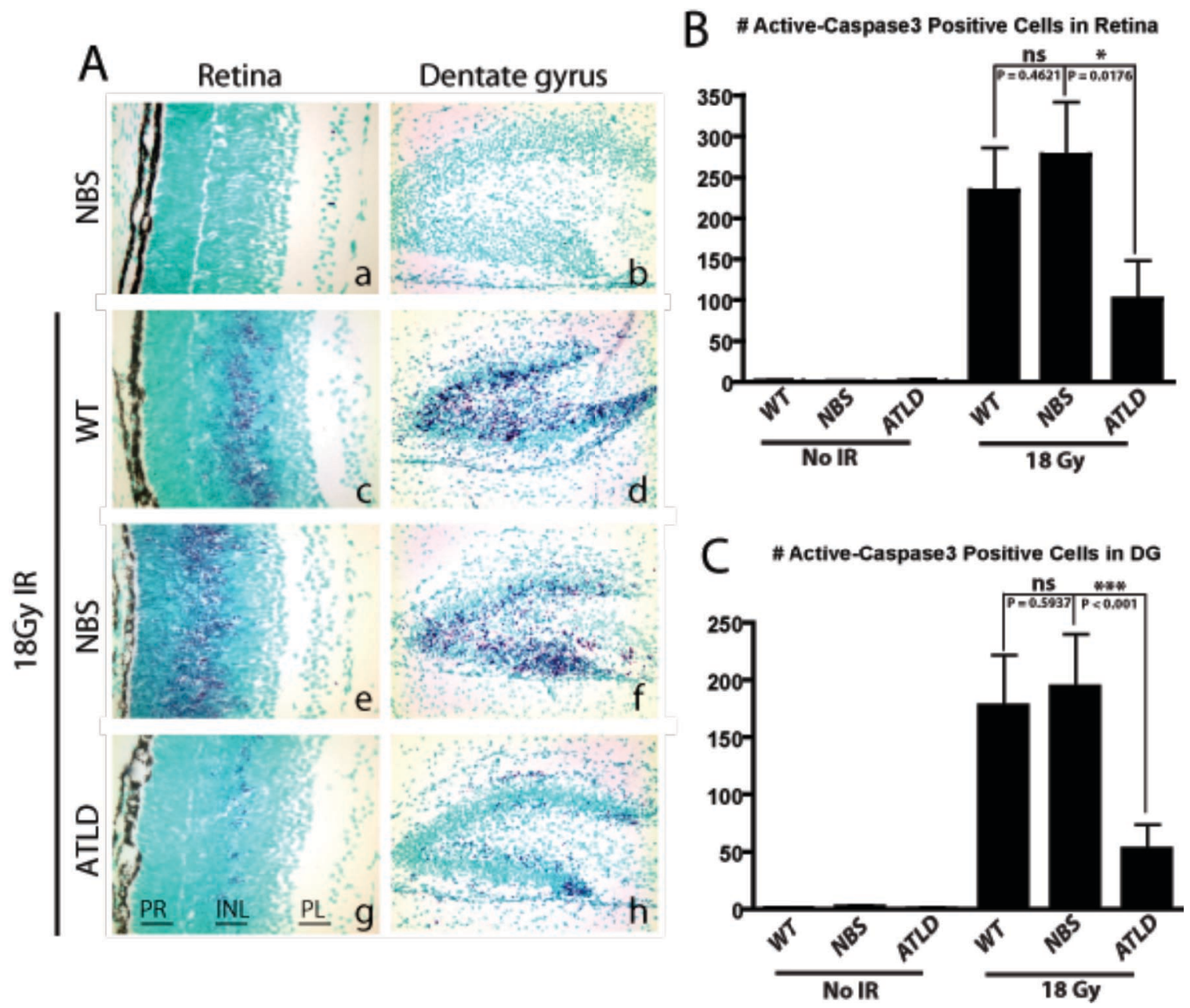

Figure 2.3 Radiation-induced apoptosis in $\mathrm{Mre}^{\mathrm{ATLD1/ATLD1}}$ and $\mathrm{Nbs} \mathbf{1}^{4 B / A B} \mathrm{CNS}$. (A) WT, Mre $11^{A T L D 1 / A T L D 1}$ and $N b s 1^{\triangle B / \triangle B}$ mice were treated with $8 \mathrm{~Gy}$ of $\gamma$-irradiation and collected $6 \mathrm{hr}$ post-IR. Immunostaining was performed against active-caspase 3 . Images of the retina ( $\mathrm{a}, \mathrm{c}, \mathrm{e}, \mathrm{g})$ are at 400x magnification. Images of the dentate gyrus $(\mathrm{b}, \mathrm{d}, \mathrm{f}, \mathrm{h})$ are at 200x magnification. Retinal photoreceptor layer (PR), inner nuclear layer (INL), plexiform layer (PL) are indicated. Arrows point to immunopositive cells. (B) Quantitation of the total number of active-caspase 3 positive cells in the retina. Error bars represent standard deviation. (C) Quantitation of the total number of active-caspase 3 positive cells in the dentate gyrus. Error bars represent standard deviation. 
Figure 2.4 DNA damage responses in the $M r e 11^{A T L D 1 / A T L D 1}$ and $N b s 1^{A B / A B}$ nervous systems.

(A) WT, Mre $11^{A T L D I / A T L D 1}$, and $N b s 1^{\triangle B / \triangle B}$ mice were treated with indicated doses of $\gamma$ irradiation and cerebella were harvested 30 min post-IR. Total Atm was

immunoprecipitated from cerebellar lysates followed by immunoblotting against Atm pSer1987. Total Atm served as the loading control. Corrections were made for loading by normalization of Atm pSer1987 bands to total Atm. Quantitative analysis depicted under the Atm pSer1987 bands. (B) WT, Mre $11^{A T L D 1 / A T L D 1}$, and $N b s 1^{\triangle B / A B}$ mice were treated with indicated doses of $\gamma$-irradiation and cerebella were harvested $3 \mathrm{hr}$ post-IR. Cerebellar lysates were probed using antibodies recognizing Nbs1, Mre11 and Chk2. Phosphorylated Chk2 (pChk2) was detected as a mobility shift. $\beta$-actin was the loading control. (C) WT, Mre $11^{A T L D I / A T L D 1}$, and $N b s 1^{\triangle B / \triangle B}$ mice were treated with 18 Gy of $\gamma$ irradiation and sacrificed $4 \mathrm{hr}$ post-IR. Immunostaining was performed against p53phosphoserine 18 and active-caspase 3. Micrographs depict cerebellum and are at 200x magnification. (D) WT, $\mathrm{Atm}^{-/}$and Mre $11^{A T L D I / A T L D 1}$ were treated with 4Gy of IR to determine Chk2 and p53 phosphorylation in $\mathrm{Atm}^{-/}$cerebellum. (E) WT, Mre $11^{\text {ATLD I/ATLD1 }}$ and $N b s 1^{\Delta B / \Delta B}$ mice were treated with $18 \mathrm{~Gy}$ of $\gamma$-irradiation and collected $3 \mathrm{hr}$ post-IR. Cerebellar lysates in D and E were probed for Chk2, p53 (pSer18) and Nbs1 or Mre11. Ponceau staining was used to determine equal loading. The p53(s18) levels were normalized to the upper band of the Ponceau stain, as indicated by numbers above the bands. 

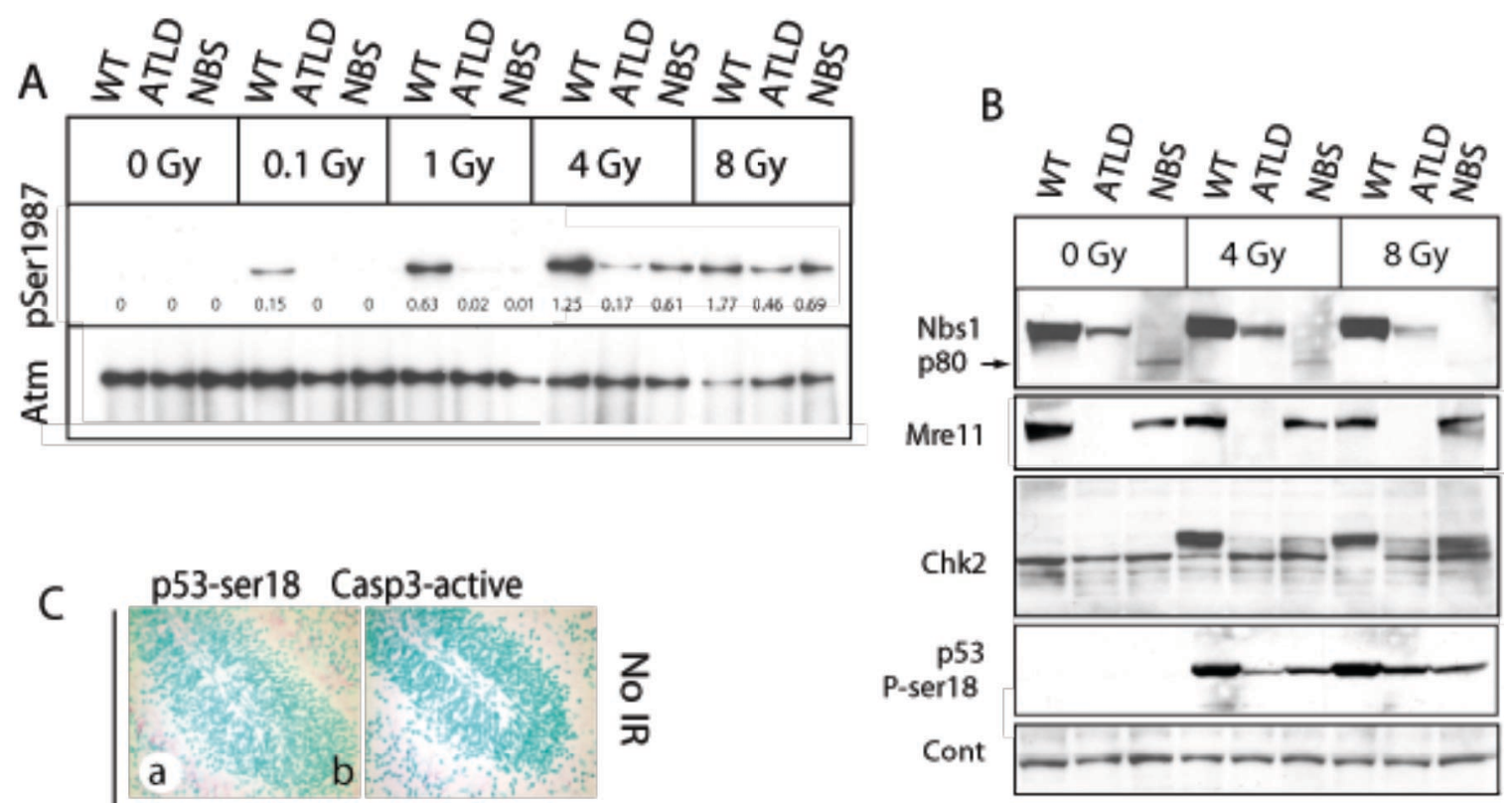

$\frac{z}{\overline{0}}$
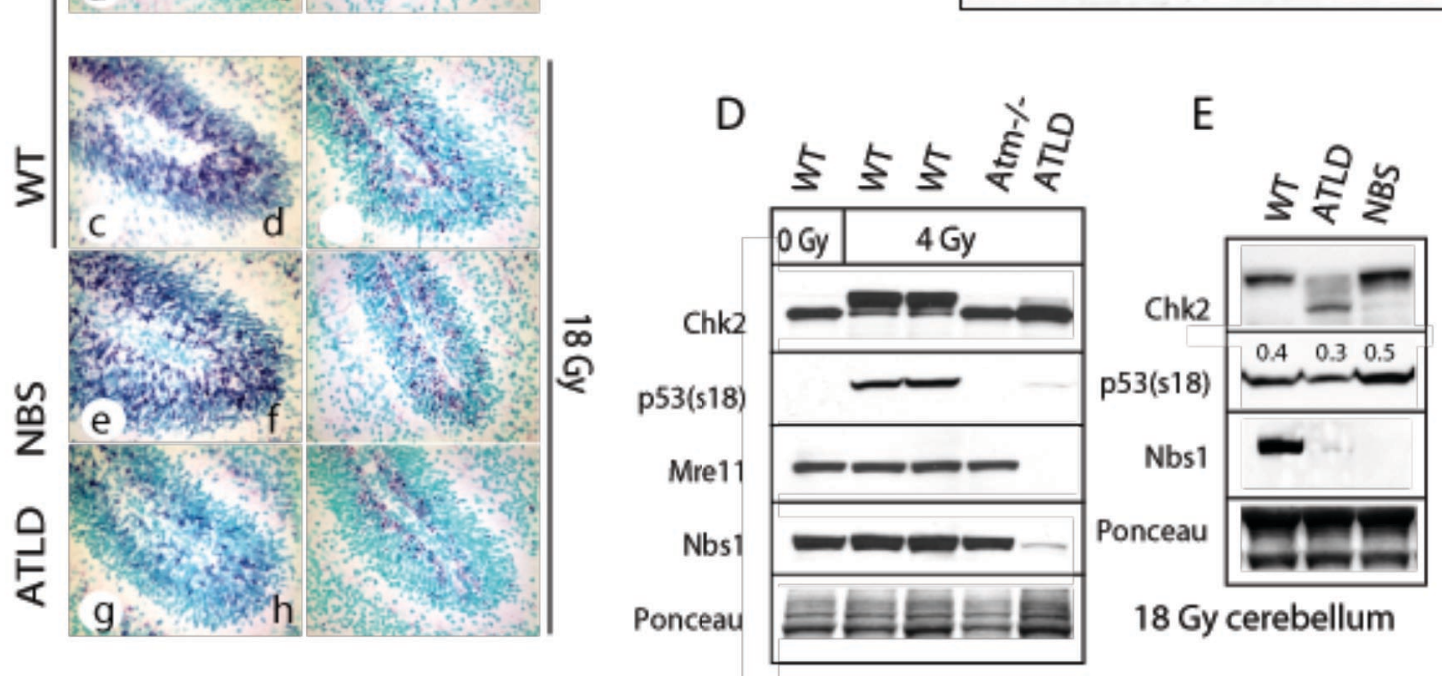
full length $\mathrm{Nbs} 1$ was not present in the $N b s 1^{\Delta B / A B}$ samples (Figure 2.4B), although he did detect the $80 \mathrm{kDa}$ product translated from the $\mathrm{Nbs} 1$ mutant message (Maser et al., 2001). Similarly, the COOH-terminus of Mre11 was not detected in Mre $11^{\text {ATLDI/ATLDI }}$ tissue and levels of Nbs1 were also substantially reduced (Figure 2.4B). Consequently, we determined other readouts of Atm signaling relevant to apoptosis. Chk2 is a key DNA damage signaling effector that is activated by Atm-dependent phosphorylation (Ahn et al., 2000; Hirao et al., 2000; Matsuoka et al., 2000), and is essential for radiation-induced apoptosis in the developing nervous system (Takai et al., 2002). In both Mre $11^{\text {ATLD1/ATLDI }}$ and $N b s 1^{\triangle B / \Delta B}$ tissues, Mr. Zhao found that there was clearly less Chk2 modification compared with WT after irradiation. However, despite this, Chk2 activation was substantially greater in the $N b s 1^{\Delta B / \triangle B}$ tissue compared to Mre $11^{A T L D 1 / A T L D 1}$ particularly after 8Gy (Figure 2.4B). We also evaluated ser-18 phosphorylation of p53 (equivalent to serine-15 of human p53) after radiation of P5 cerebellum using immunohistochemistry and western blot analysis. While both WT and $N b s 1^{\Delta B / A B}$ tissue had robust induction of p53 ser18 phosphorylation and caspase-3 activation, both were markedly reduced in Mre $11^{A T L D 1 / A T L D 1}$ tissue (Figure 2.4C). Furthermore, Mr. Zhao also determined the relative level of Chk2 activation in $\mathrm{Atm}^{-/-} \mathrm{P} 5$ cerebellum, as a comparison to the MRN mutants, and found that no Chk 2 activation or p53ser 18 phosphorylation occurred after 4Gy of IR, consistent with apoptosis being Atm-dependent in this tissue (Figure 2.4D). Additionally, he found that at higher radiation doses (18Gy) the difference between Chk2 activation in Mre $11^{A T L D I / A T L D 1}$ and $N b s 1^{\triangle B / \triangle B}$ is more apparent, as is, albeit to a lesser degree, phosphorylation of p53ser18 (Figure 2.4E). The abundant apoptosis and Chk2 modification observed in the $N b s 1^{\Delta B / \triangle B}$ mice indicate that the hypomorphic Nbs1 allele can activate Atm sufficiently to engage apoptosis in the CNS after DNA damage. We also observed defects in Atm signaling in the Mre $11^{A T L D 1 / A T L D 1}$ and $N b s 1^{\Delta B / \triangle B}$ thymus, with reduced apoptosis in ATLD tissue (data not shown), consistent with recent data using Mre $11^{A T L D I / A T L D 1}$ thymocytes (Stracker et al., 2008). Thus, the MRN complex is required for ATM activation, and this is defective in both Mre $11^{A T L D 1 / A T L D 1}$ and $N b s 1^{\triangle B / \triangle B}$ tissue. However, the Mre $11^{A T L D 1 / A T L D 1}$ mutation results in a more substantial block in Atm activation that includes abrogation of apoptosis.

\subsubsection{Characterization of $\operatorname{Lig} 4^{\text {Nes-cre }}$ mice}

The Lig ${ }^{\text {Nes-cre }}$ animal model was generated to be used as a tool in which to study the chronic consequences of endogenously-occurring DNA damage in the nervous system. Dr. Helen Russell generated the mice through restriction enzyme-mediated cloning and performed southern and northern blots using DNA extracted from various tissues of embryonic to P5 aged mice to confirm efficiency and specificity of gene deletion in the Nestin-cre background. I used real-time PCR analysis to verify deletion of the Lig4 open reading frame in the E13.5 CNS (Figure 2.5).

Lig $4^{\text {Nes-cre }}$ mice were born at the expected frequency and were viable into adulthood. Initial histological analysis revealed pronounced microcephaly, but largely normal cellular and gross morphology (Figure 2.6). Though apparently healthy, it was 


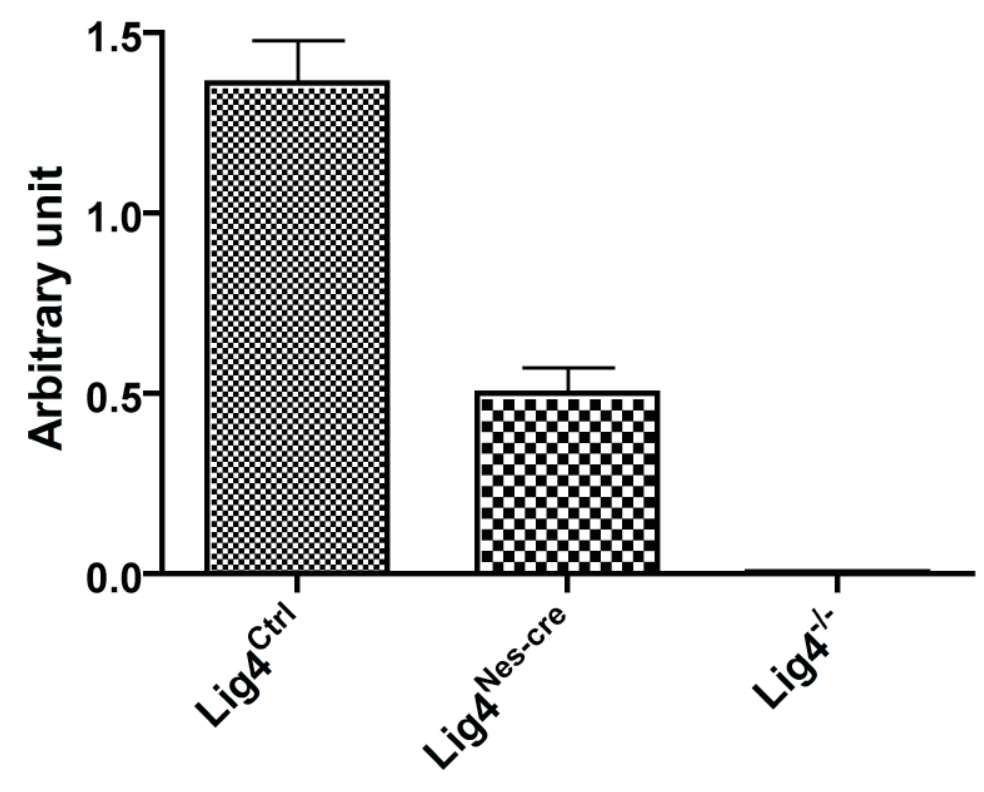

Figure 2.5 Real-time PCR demonstrating Lig4 gene excision in E13.5 Lig4 ${ }^{\text {Nes-cre }}$ CNS. Embryonic brain and spinal was isolated from cryosections. DNA was phenol/chloroform extracted. Error bars represent standard deviation between duplicate results using DNA from the same sample.
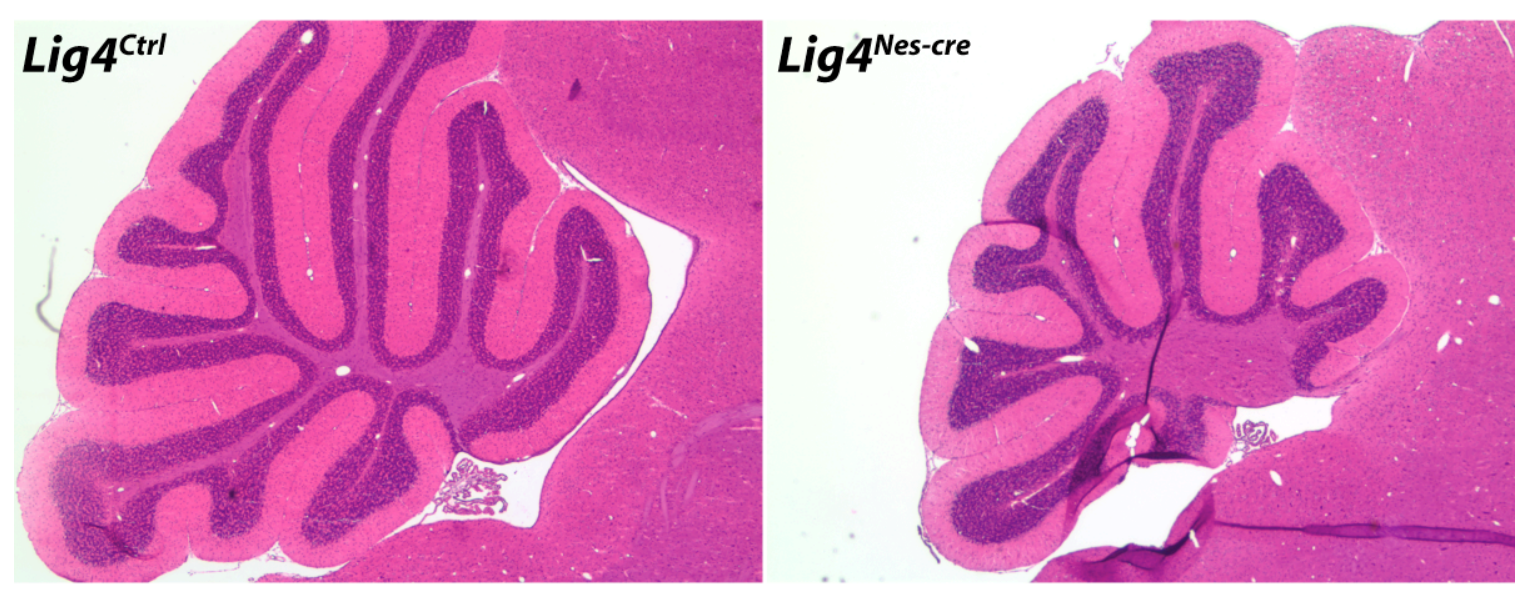

H\&E (2.5x)

Figure 2.6 Diminished cerebellar size reflective of overall microcephaly in Lig $4^{\text {Nes-cre }}$ mice.

Five month old $\mathrm{Lig} 4^{\mathrm{Ctrl}}$ and $\mathrm{Lig} 4^{\text {Nes-cre }}$ mice were transcardially perfused and frozen sections (10 $\mu \mathrm{m}$ thick) were stained for hematoxylin and eosin (H\&E). Photomicrographs were taken at $25 \mathrm{x}$ magnification. 
noticed that $\operatorname{Lig} 4^{\text {Nes-cre }}$ females tended to produce small litters often with less than 5 pups and ceased to efficiently reproduce around 4 months of age. In addition, two Lig $4^{\text {Nes-cre }}$ males that were placed with healthy $\operatorname{Lig} 4^{+/ l o x P}$ females not expressing cre failed to mate. Notably, around 4 months of age these animals began to appear vaguely unhealthy (i.e., rough coat and lethargic). Between 4-6 months of age all Lig4 ${ }^{\text {Nes-cre }}$ began to develop mild hindlimb ataxia most noticeable as loss of balance that progressed to an inability to move the hindlimbs forward such that dragging the legs was the only means of mobility. Full hindlimb paralysis was not observed either because the pathology had not progressed to its full extent at the time of euthanasia or the mechanism behind this phenomenon failed to cause paralysis. This phenotype was observed in all Lig $4^{\text {Nes-cre }}$ animals aged to at least 1 year (at least 20 animals) and was not seen in an equal number of age-matched controls. Despite dramatic phenotypic penetrance, no neuropathology was found that would account for this presentation. No degeneration was observed in adult Lig4 ${ }^{\text {Nes-cre }}$ brain or spinal cord based on TUNEL and FluoroJade C staining (data not shown). Presentation appeared consistent with spinal cord damage, thus analysis of the vertebrae and spinal cord was performed. In conjunction with the St. Jude Children's Research Hospital Animal Imaging Center, CT scans were taken of the entire vertebral column of Lig ${ }^{\text {Nes-cre }}$ mice that exhibited severe hindlimb impairment and age-matched controls to determine if bone malformations might have caused compression of the spinal cord (Figure 2.7). No evidence of such occurrence was observed (Personal communication with Dr. Chris Calabrese, April 18, 2007).

Histology of the lumbar spinal cord was performed. Toluidine blue staining was used to assess white matter tracts and Nissl stain was used to look at neuronal soma. Neither stain revealed evidence of pathology (data not shown). Similarly, H\&E staining failed to detect degenerative changes in the ig $^{\text {Nes-cre }}$ spinal cord and immunostaining against myelin basic protein showed normal patterns of myelination compared to agematched and sex-matched $\mathrm{Lig}^{\mathrm{Ctrl}}$ samples (Figure 2.8). Cellularity did appear to be decreased in the spinal cord, based upon H\&E staining. This was not confirmed through quantitation and likely reflects cell loss during development. All panels were taken at the same magnification. $\mathrm{H} \& \mathrm{E}$ panels taken at low magnification demonstrate that similar to microcephaly observed in the Lig $^{\text {Nes-cre }}$ mice, the spinal cord is much smaller compared to controls.

Though Lig4 $4^{-1-}$ embryos exhibited extensive Atm/p53-dependant apoptosis in the nervous system prior to death, it has not been formally shown (though assumed) that the accumulation of unrepaired DSBs were the reason for cellular demise. The Lig $4^{\text {Nes-cre }}$ mice were valuable as a tool with which the inherent occurrence and consequences of DNA damage could be observed in the mammalian nervous system from embryonic life through adulthood. Similar to the DNA damage response described in astrocytes, immunostaining against $\gamma-\mathrm{H} 2 \mathrm{AX}$ and 53BP1 was used to track the appearance and resolution of DNA damage. Failed attempts were made at performing pulsed-field gel electrophoresis directly upon tissue samples to corroborate immunostaining (data not shown). Foci were rarely observed in $\mathrm{Lig}^{\mathrm{Ctrl}}$ animals and typically appeared only in a few cells throughout the entire brain (Figures 2.9-2.11). In those few cells, less than five foci were typically observed. Spatiotemporal mapping of foci in $\operatorname{Lig} 4^{\text {Nes-cre }}$ mice revealed 

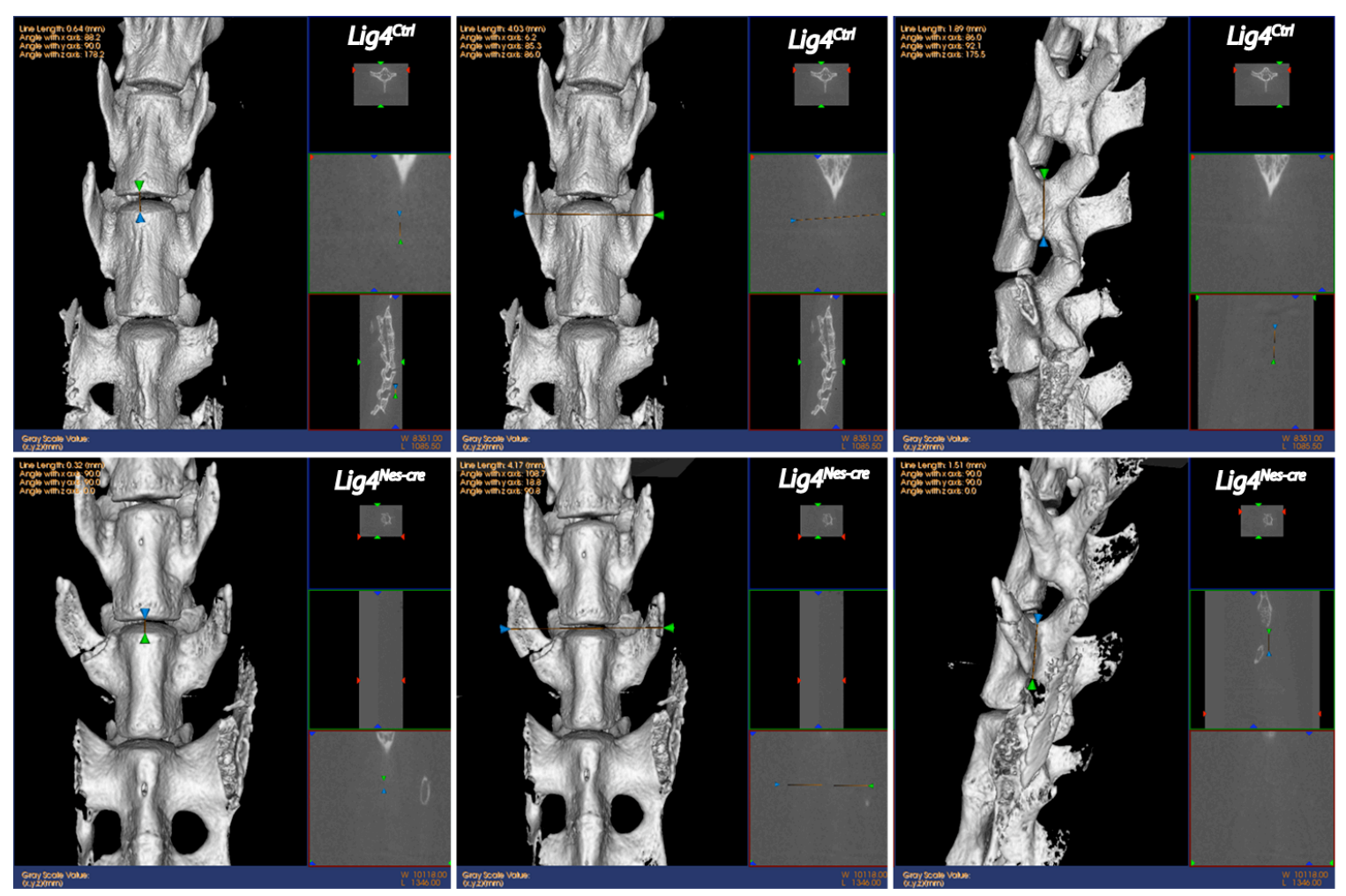

Figure 2.7 CT scan of spinal column collected from Lig $4^{\text {Nes-cre }}$ mouse with end-stage hindlimb ataxia and age-matched $\operatorname{Lig} 4^{\mathrm{Crrl}}$.

Personal communication with Dr. Chris Calabrese, April 18, 2007. 
Lig4 ${ }^{\text {Ctrl }}$

Lig4 $4^{\text {Nes-cre }}$
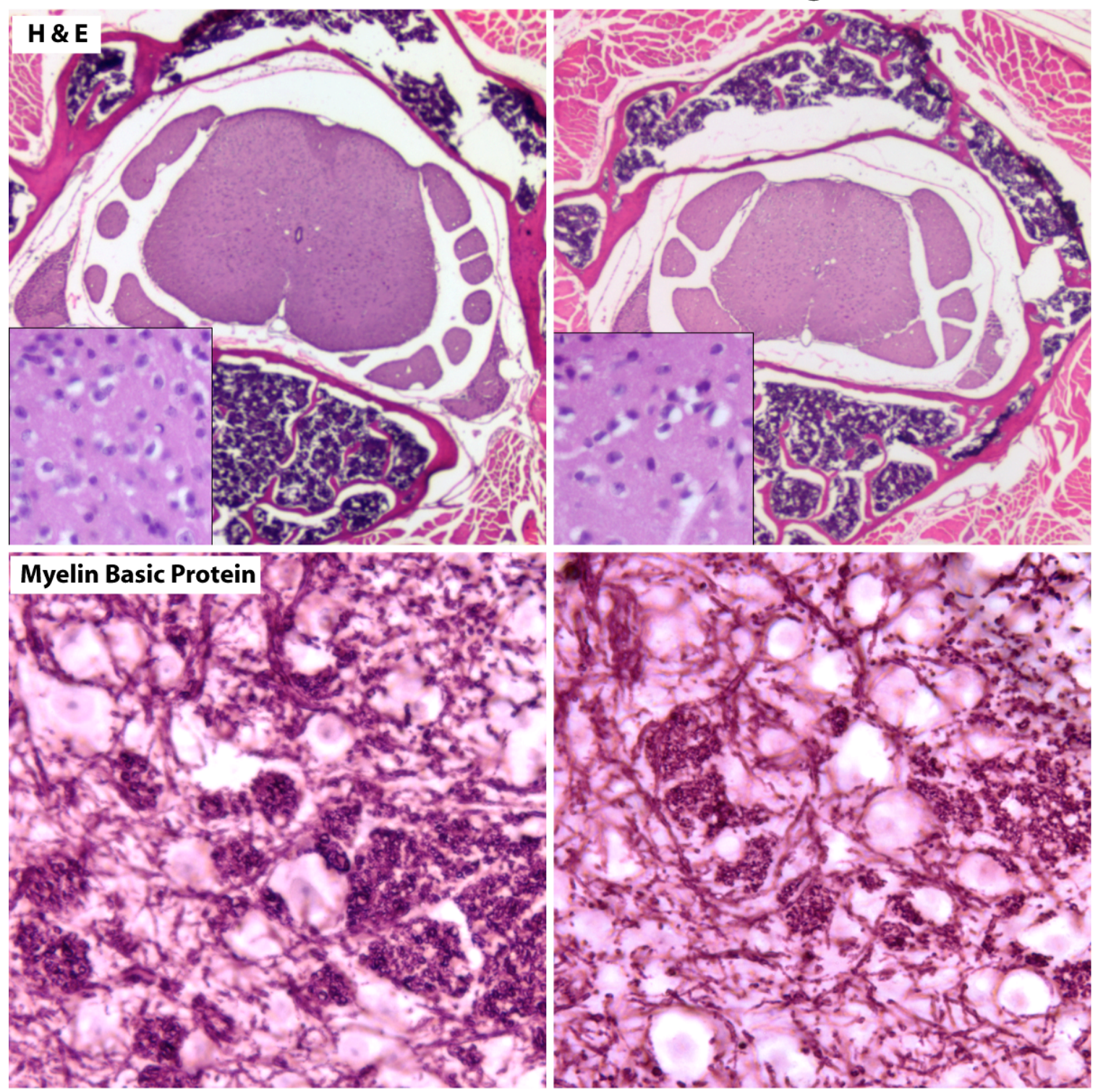

Figure 2.8 Histology observed in Lig $4^{\text {Nes-cre }}$ spinal cord.

Upper panels show H\&E stained paraffin sections of 12 month old Lig $4^{\mathrm{Crrl}}$ and Lig $4^{\text {Nes-cre }}$ spinal cord (25x magnification). Insets of the dorsal horn are shown in the lower left corner of each panel (200x magnification). Lower panels show dorsal horn of 12 month old Lig4 ${ }^{\mathrm{Crrl}}$ and $\mathrm{Lig} 4^{\mathrm{Nes}-\mathrm{cre}}$ spinal cord immunostained against myelin basic protein (400x magnification). 


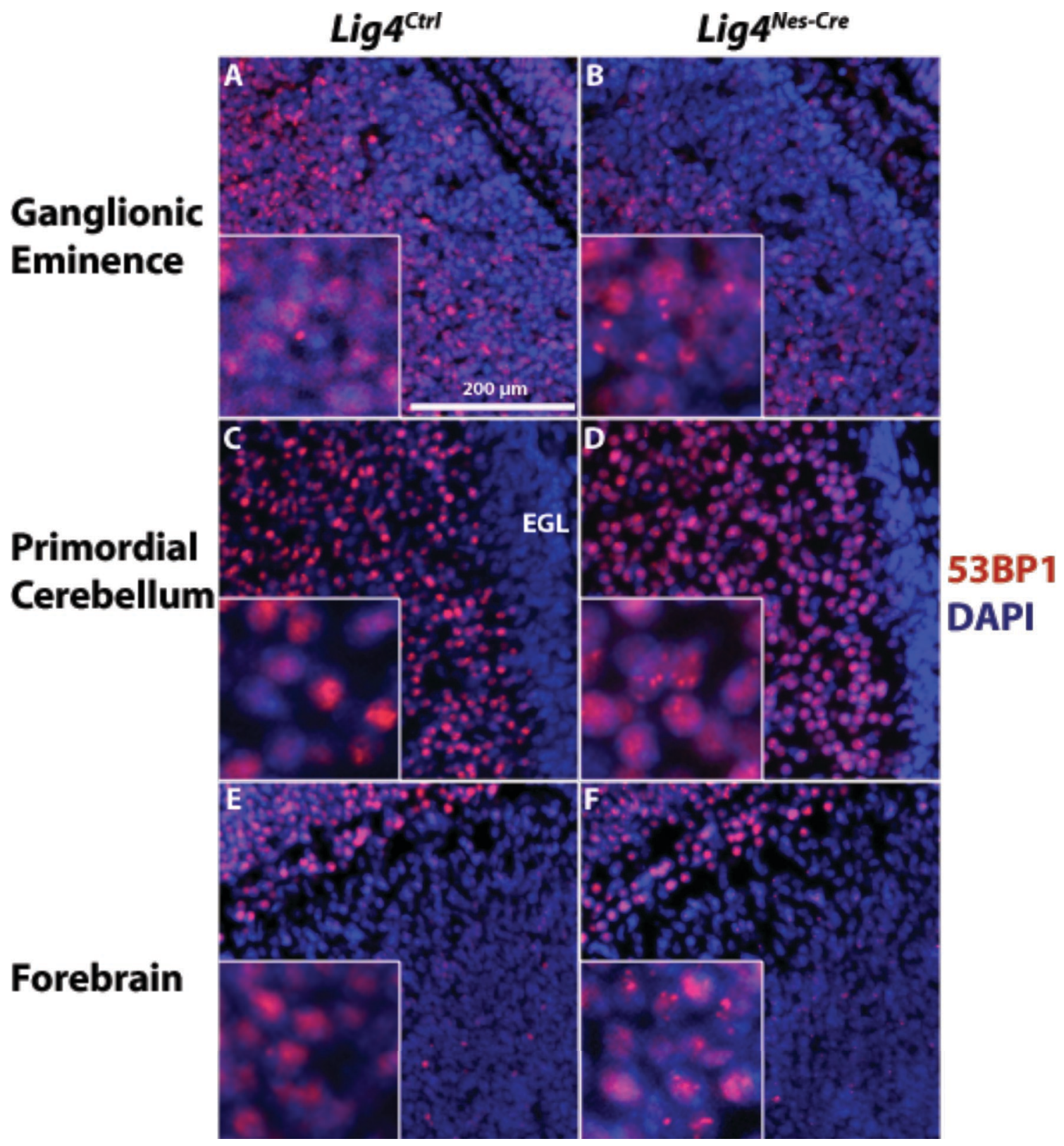

Figure 2.9 Pattern of 53BP1 foci in E15.5 Lig $4^{\text {Nes-cre }}$ brain.

53BP1 were observed in the indicated areas of the Lig $4^{\text {Nes-cre }}$ embryonic brain. Agematched $\operatorname{Lig} 4^{\mathrm{Ctrl}}$ samples showed diffuse nuclear staining of 53BP1 (A, C, E insets) rather than punctuate foci (B, D, F insets). Photomicrographs were captured at 200x magnification. 


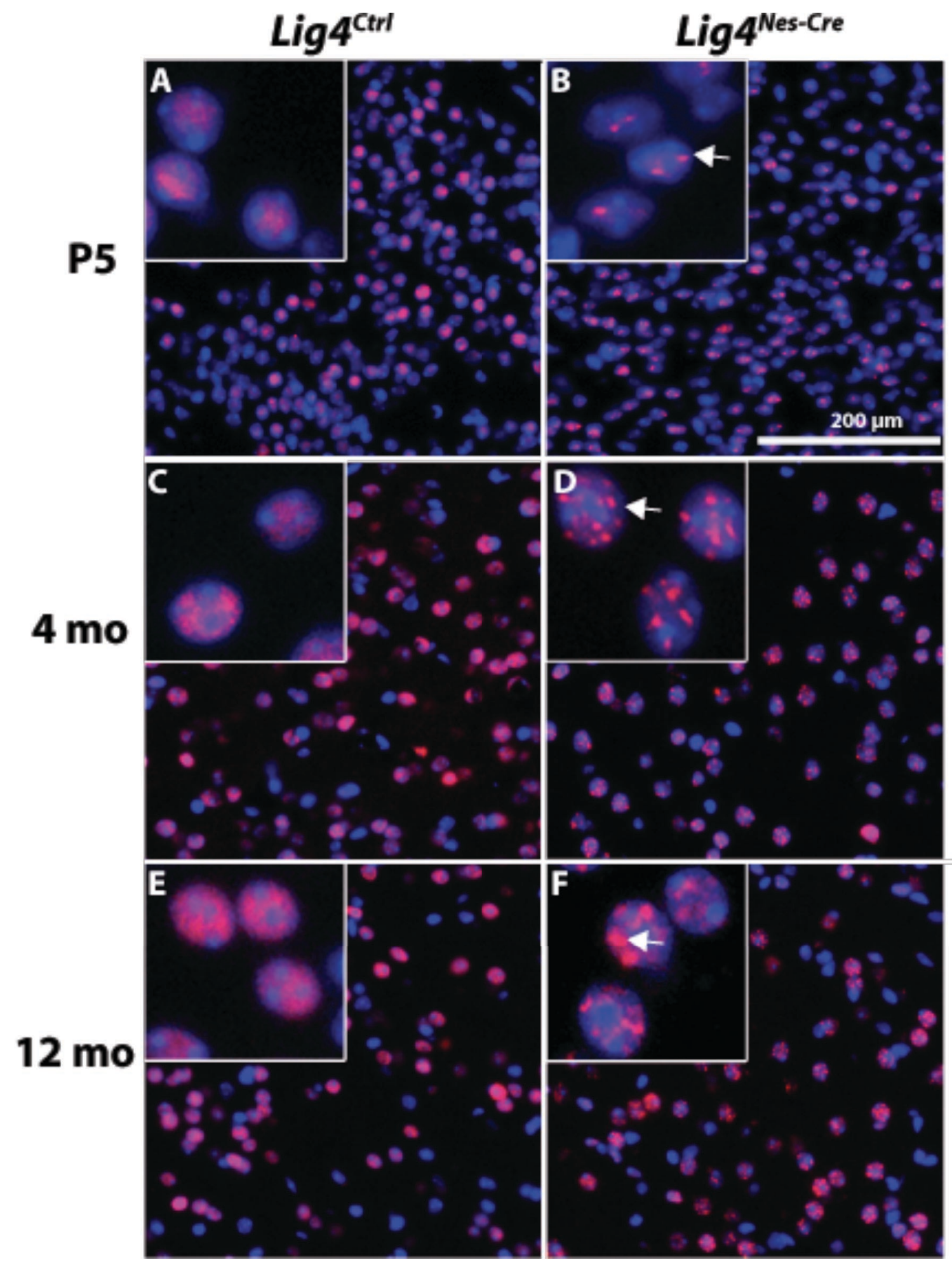

Figure 2.10 Comparison of 53BP1 foci in caudate putamen of P5, 4 month old, and 12 month old Lig4 ${ }^{\text {Nes-cre }}$ mice.

Photomicrographs were captured at 200x magnification. Arrows indicate distinct foci. 


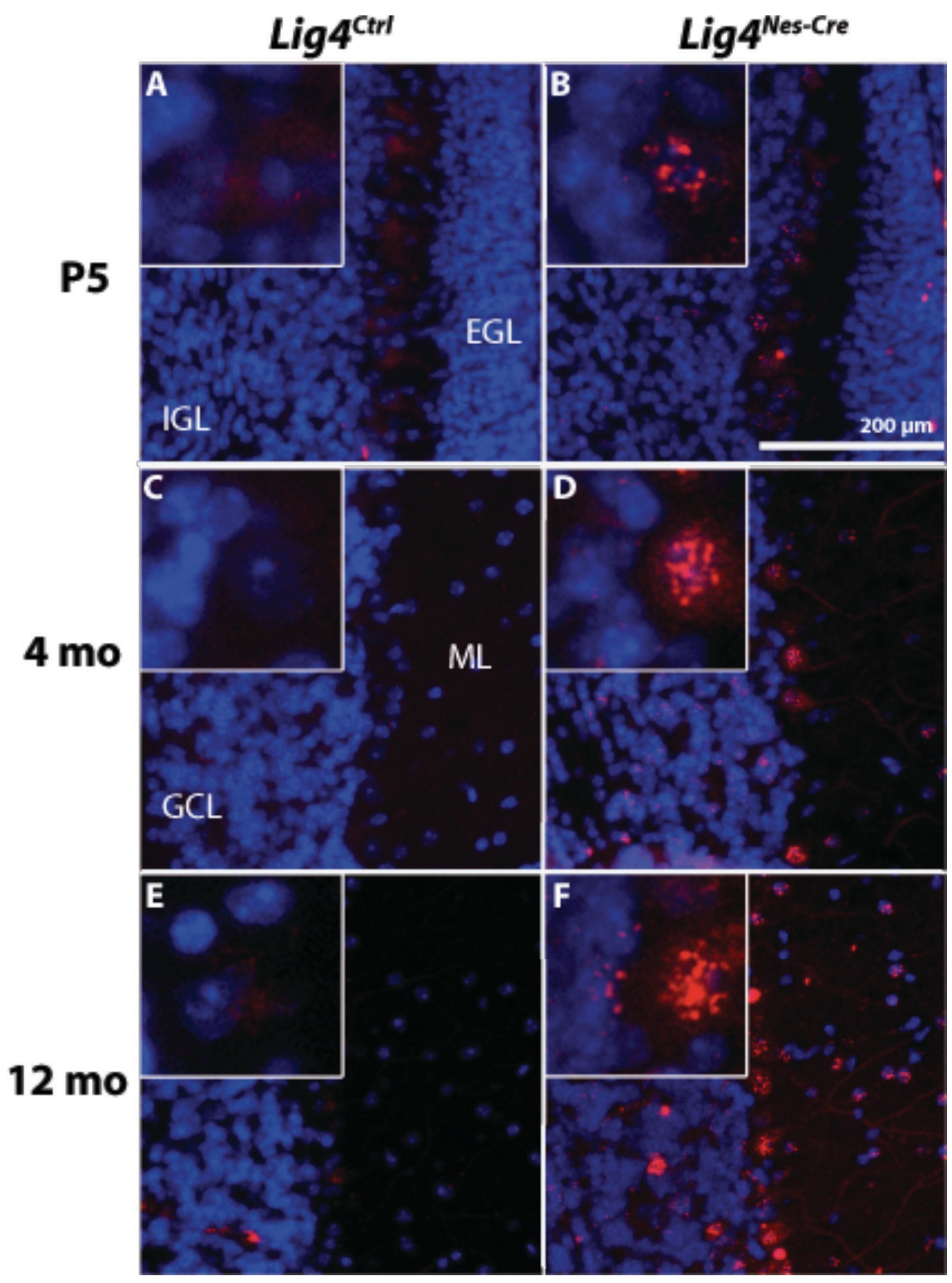

Figure 2.11 Comparison of $\gamma-\mathrm{H} 2 \mathrm{AX}$ expression in cerebella of $\mathrm{P} 4,4$ month old, and 12 month old Lig $4^{\text {Nes-cre }}$ mice.

Photomicrographs were captured at 200x magnification. 
that DNA DSBs arise during embryogenesis and continue to accumulate during adulthood (up to 1 year of age) in the brain (Figures 2.9-2.12). Semi-quantitative mapping throughout major regions of the postnatal and adult Lig $4^{\text {Nes-cre }}$ brains revealed that there is no striking regional or cell-type specificity in which damage occurs.

While endogenous DNA repair foci allowed us to see where damage occurs naturally in the brain, it provided only a snapshot in time of existing damage without indication of whether DSBs were being left unrepaired and accumulating, or if endogenous damage was occurring with a higher frequency in older animals. Given the role of Lig4 in NHEJ, 53BP1 and $\gamma$-H2AX foci observed endogenously in the Lig $4^{\text {Nes-cre }}$ mice most likely represent the former. To ascertain the likelihood of this, repair capacity was measured in vivo through irradiation and prolonged recovery of age-matched Lig $4^{\text {Nes- }}$ ${ }^{c r e}$ and $\mathrm{Lig} 4^{\mathrm{Ctrl}}$ mice followed by immunostaining against 53BP1 (data not shown) and $\gamma-$ H2AX (Figure 2.13). The results of this experiment indicated that little or no repair occurred in the Lig $^{\text {Nes-cre }}$ brain up to 7 days post-IR, further suggesting that no significant back-up method of NHEJ exists in the nervous system.

In summary, the Lig4 ${ }^{\text {Nes-cre }}$ mutant is a viable mouse model of DNA ligase IV deficiency in the nervous system. Though microcephalic, these mice do not exhibit any other notable neuropathology. Microcephaly is likely attributable to increased apoptosis during neurogenesis, due to an overload of DNA DSBs in nascent post-mitotic cells. Despite the overwhelming presence of DNA damage in the adult Lig $4^{\text {Nes-cre }}$ brain, apoptosis is not induced (data not shown). Thus, the unexplained hindlimb ataxia is difficult to directly relate to DNA damage, though how the accumulation of DNA DSBs affects function of viable neurons is unknown.

\subsubsection{Microcephaly after Lig4 loss requires Atm signaling}

In comparison to age-matched and sex-matched littermates $\mathrm{Lig}^{\mathrm{Ctrl}}\left(\mathrm{Lig}^{+/ l o x P}\right.$; Nestin-cre or Lig $\left.4^{\text {loxP/loxP }}\right), \operatorname{Lig} 4^{\text {Nes-Cre }}$ animals exhibit pronounced microcephaly as evident by an approximately $40 \%$ reduction in brain size (Figure 2.14). Notably, microcephaly is a characteristic of LIG4 syndrome patients with hypomorphic mutations in LIG4 (O'Driscoll et al., 2004). To determine the cause of the microcephaly in the $\mathrm{Lig} 4^{\mathrm{Nes}-\mathrm{Cre}}$ mice we assessed apoptosis at various developmental stages, and found that from E13.5 onwards there was markedly increased apoptosis in Lig $4^{\text {Nes-Cre }}$ neural tissue as judged by TUNEL and caspase 3 staining (Figure 2.15), with a peak around E15.5 somewhat similar to germline disruption of Lig4. Thus, microcephaly in $\mathrm{Lig} 4^{\mathrm{Nes}-\mathrm{Cre}}$ mice likely results from apoptotic cell loss during development. Therefore we generated

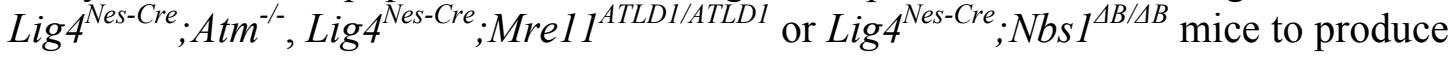
viable mice with which to monitor longer term consequences of MRN mutations. Initially, we confirmed that both MRN mutations produced effects in the Lig $4^{\text {Nes-cre }}$ mice similar to those we observed after germ line disruption of Lig4. Apoptosis was abrogated in $\mathrm{Lig} 4^{\mathrm{Nes}-\mathrm{Cre}}$;Mre $11^{\text {ATLD1/ATLD1 }}$ and Lig4 ${ }^{\mathrm{Nes}-\mathrm{Cre}} ; \mathrm{Atm}^{-/-}$but occurred to the same level as Lig ${ }^{\text {Nes-Cre }}$ in $\mathrm{Lig}^{\mathrm{Nes}-\mathrm{Cre}} ; \mathrm{Nbs} 1^{\triangle B / \Delta B}$ mice (Figure 2.15). To assess the long-term 


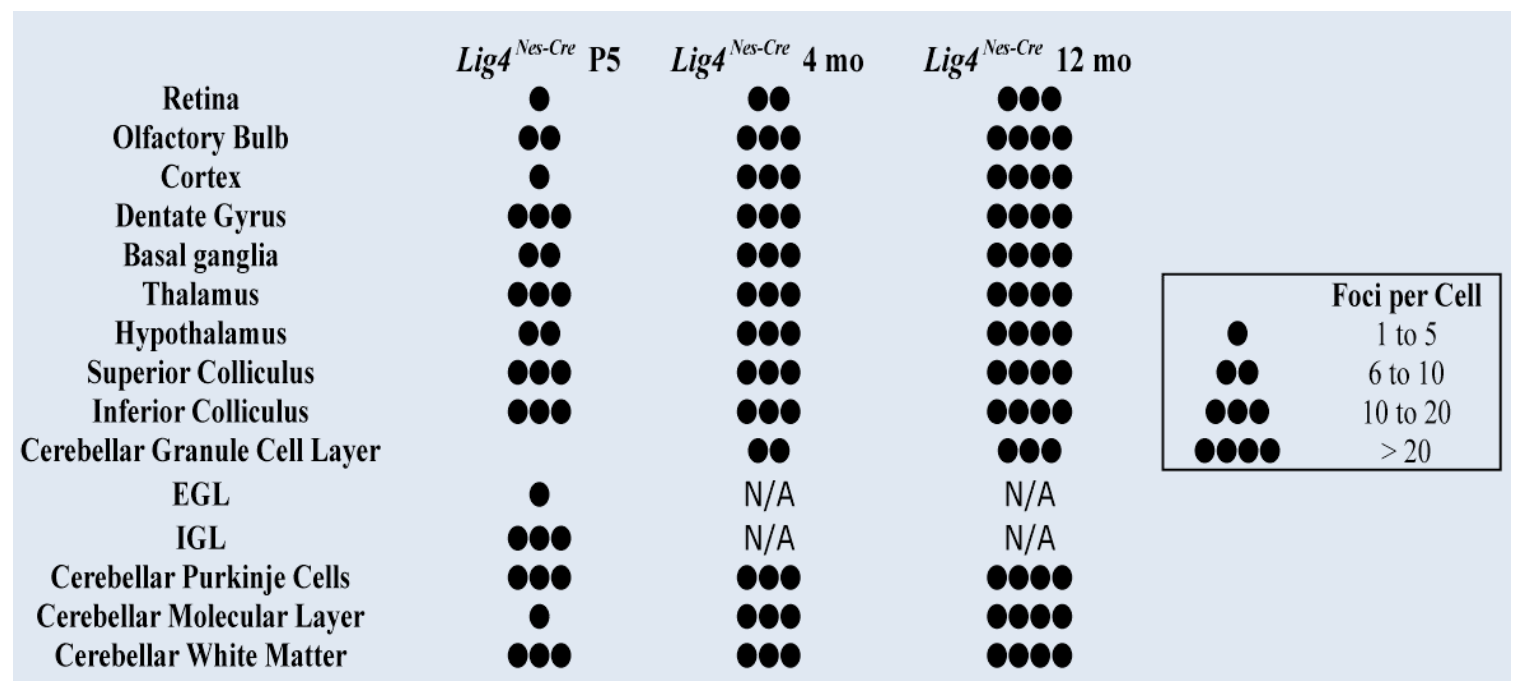

Figure 2.12 Spatiotemporal mapping of 53BP1 and H2AX foci in Lig $4^{\text {Nes-cre }}$ brains. Foci were semi-quantitated manually at 400x magnification in the indicated regions of the brain at the indicated ages in Lig4 ${ }^{\text {Ctrl }}$ and Lig $4^{\text {Nes-cre }}$ samples. Data is shown for the Lig4 ${ }^{\text {Nes-cre }}$ samples. As pictured in Figures 2.9 and 2.10, almost no cells contained DNA either 53BP1 or $\gamma$-H2AX foci. Because the adult cerebellum contains only the granule cell layer, as the EGL no longer exists and the IGL is mature, these structures are designated as non-applicable in the $4 \mathrm{mo}$ and $12 \mathrm{mo}$ samples. That is indicated under the cerebellar granule cell layer in adult samples. Data is representative of 2 animals for each age analyzed. 


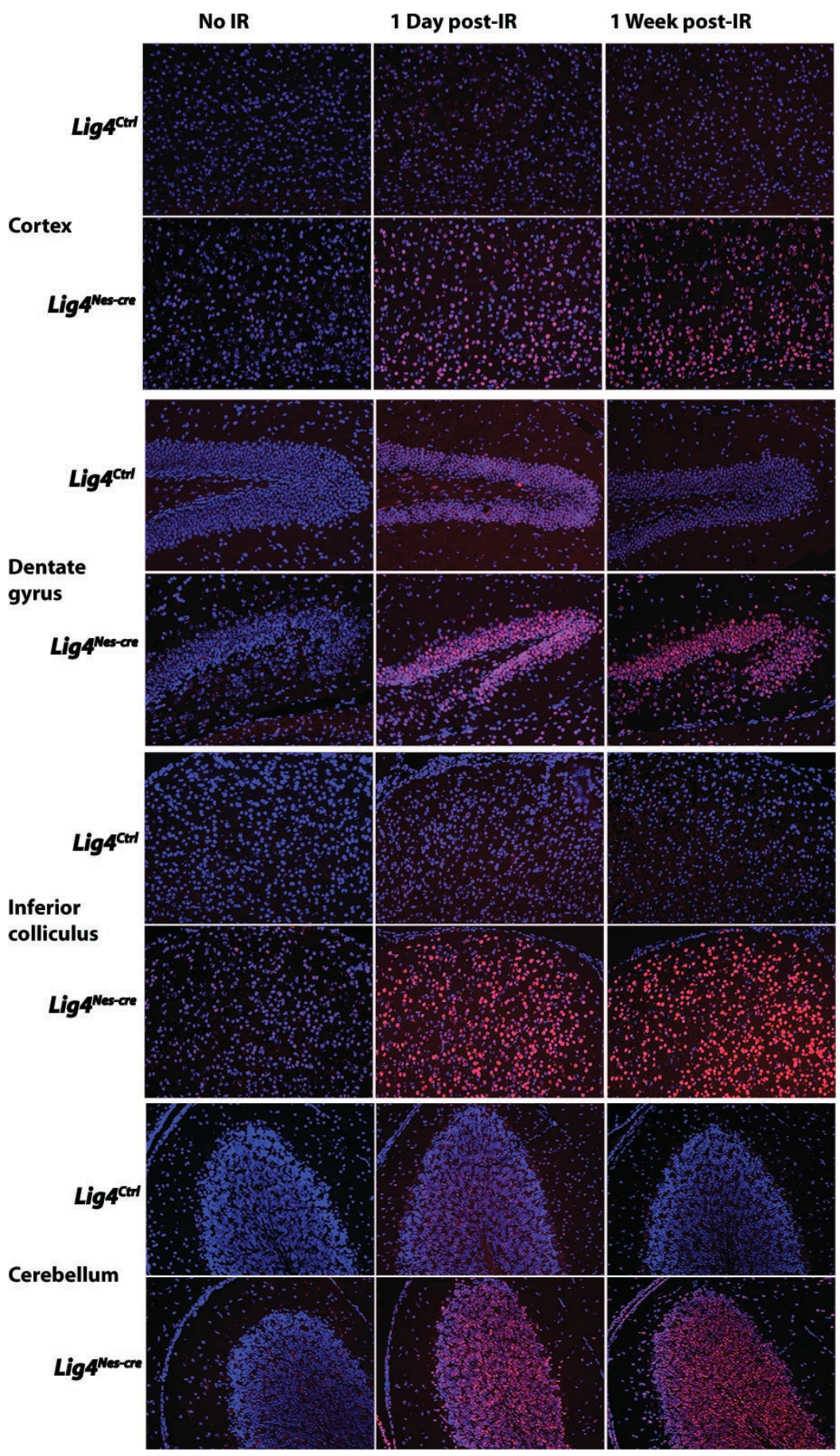

Figure 2.13 $\gamma$-H2AX foci persist in irradiated Lig $4^{\text {Nes-cre }}$ brain.

Littermate $\operatorname{Lig} 4^{\mathrm{Ctrl}}$ and Lig $4^{\text {Nes-cre }}$ mice were either untreated or irradiated with 8 Gy IR at age P21. Irradiated animals were allowed to recover for either 1 or 7 days prior to collection of brains. Immunostaining was performed against $\gamma-\mathrm{H} 2 \mathrm{AX}$ (red) and nuclei were stained with DAPI (blue). Two animals were assessed for each recovery time. Photomicrographs were captured at 200x magnification. 

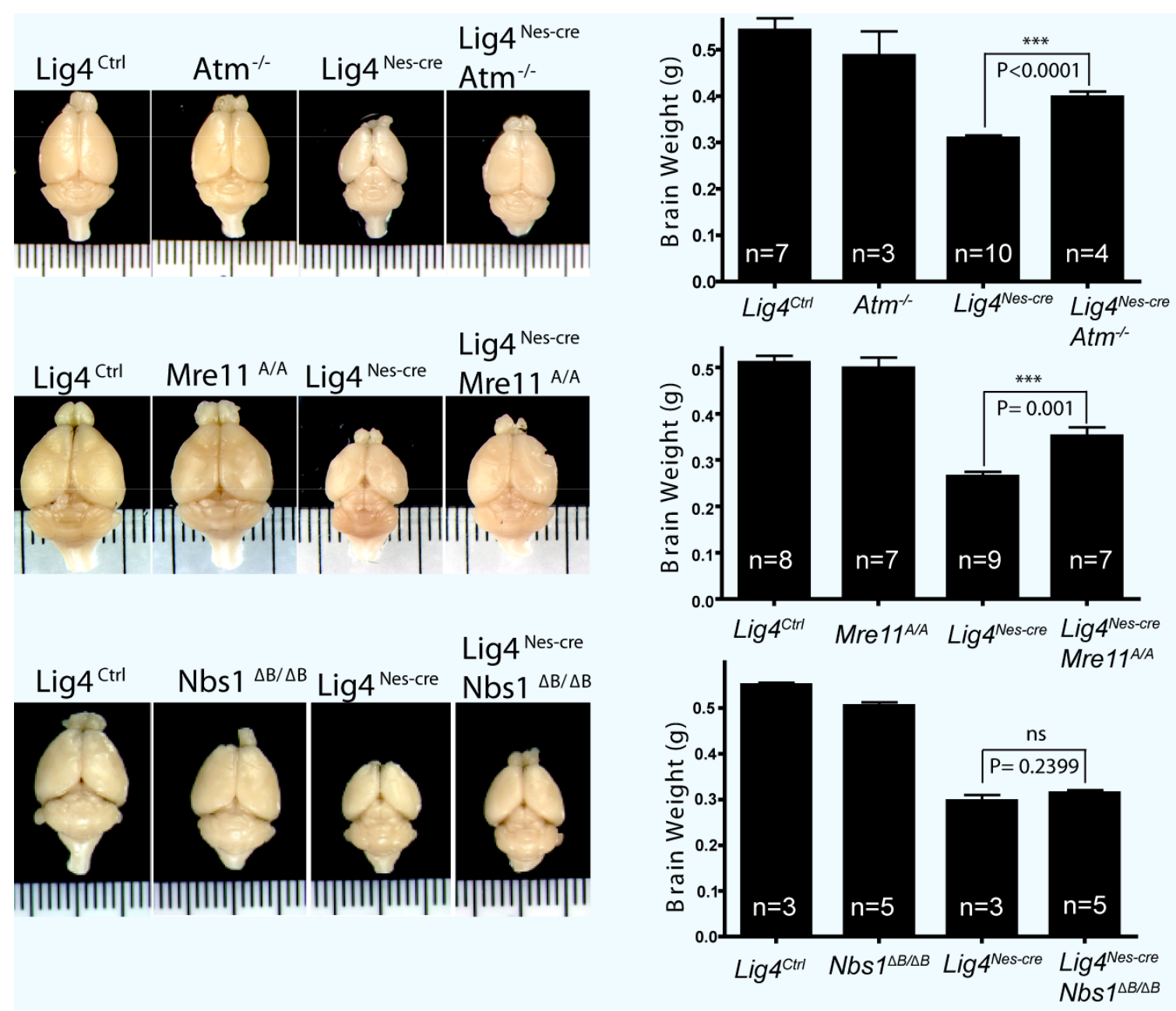

Figure 2.14 Mre11 hypomorphism and Atm loss partially rescue microcephaly in Lig4 $4^{\text {Nes-cre }}$ mice.

Measurement of A-T, A-TLD, and NBS mutations towards Lig $4^{\text {Nes-Cre }}$ brain size. Brain weights were collected from sex-matched cohorts at 4 months of age. Error bars represent standard deviation, and " $\mathrm{n}$ " is the total number of animals. 

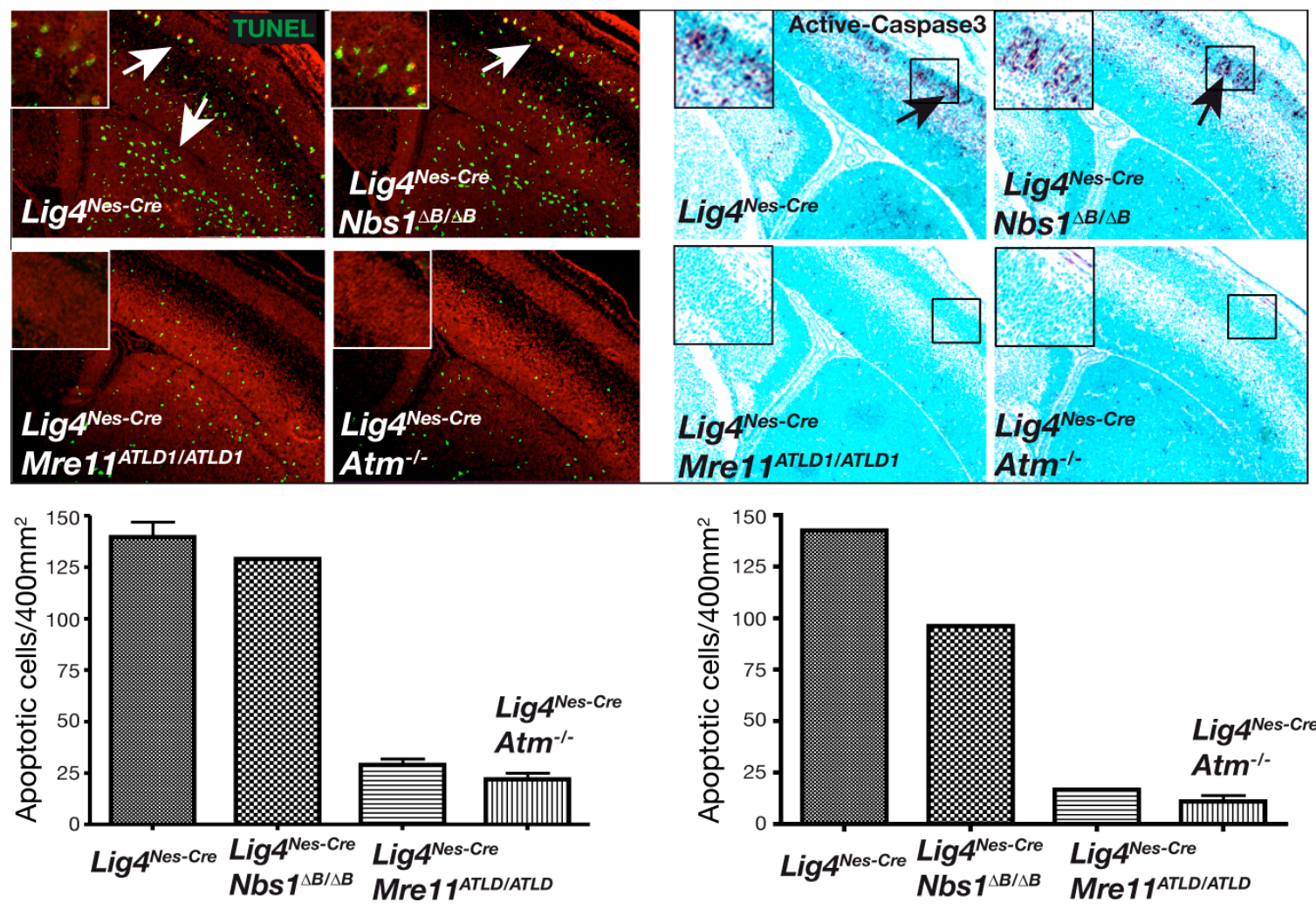

Figure 2.15 Apoptosis in E15.5 $\mathrm{Lig}^{\text {Nes-cre }}$ brain in $\mathrm{Atm}^{-/-}, \mathrm{Mre11}^{\text {ATLD1/ATLD1 }}$, and $N b s 1^{A B / A B}$ genetic backgrounds.

TUNEL analysis and immunostaining for active caspase 3 were performed on E15.5 embryos from indicated genotypes. The total number of positive cells per $400 \mu \mathrm{m}^{2}$ was quantified. Error bars represent standard deviation. Micrographs show ganglionic eminence and neopallial cortex at 200x magnification. 
consequences of MRN dysfunction and chronic DNA damage in the CNS, we monitored adult Lig4 ${ }^{\text {Nes-Cre }} ; \mathrm{Atm}^{-/-}$, Lig4 ${ }^{\text {Nes-Cre }} ;$ Mre $11^{\text {ATLD1/ATLD1 }}$ or Lig4 ${ }^{\text {Nes-Cre }} ; \mathrm{Nbs} 1^{\triangle B / \triangle B}$ mice.

Inactivation of either Atm or Mre11 significantly rescued microcephaly, whereas the $N b s 1^{\triangle B / \triangle B}$ mutation had no obvious affect towards microcephaly in Lig $4^{\text {Nes-Cre }}$ mice (Figure 2.14). Excluding microcephaly, no other significant morphologic aberrations were found in the Lig $4^{\text {Nes-Cre }}$ brain. However, with age, adult Lig $4^{\text {Nes-Cre }}$ animals developed a severe hindlimb ataxia of unknown etiology from 7-9 months onwards. Neither the $\mathrm{Atm}^{-/}$, Mre $11^{A T L D 1 / A T L D 1}$, nor $\mathrm{Nbs} 1^{A B / A B}$ background affected latency or severity of this phenotype, suggesting this later pathway is independent of MRN/ATM. Thus, the microcephalic phenotype in Lig $4^{\text {Nes-Cre }}$ mice is attributable to increased apoptosis via ATM dependent DNA damage signaling.

\subsection{Discussion}

Defective responses to DNA damage resulting from dysfunction of ATM or the MRN complex can cause the human syndromes A-T, ATLD, and NBS. Although these diseases share similar phenotypes including radiosensitivity and chromosomal instability, A-T and ATLD exhibit neurodegeneration while NBS is characterized by microcephaly. This suggests that the respective disease-causing mutations impact DNA damage signaling differently within the brain. To test this, we analyzed the DNA damage response in the nervous systems of ATLD (Mre $\left.11^{A T L D 1 / A T L D I}\right)$ and NBS $\left(\mathrm{Nbs} 1^{\triangle B / \triangle B}\right)$ mice and found that different effects upon ATM activation and downstream apoptotic signaling distinguish these MRN mutations. Therefore, we hypothesize that the different neuropathology in ATLD and NBS is a result of the relative levels of ATM activation by MRN after DNA damage, most probably in response to DNA DSBs.

While both Mre $11^{A T L D 1 / A T L D 1}$ and $N b s 1^{\triangle B / \triangle B}$ are defective in Atm phosphorylation after DNA damage, only Mre $11^{A T L D 1 / A T L D 1}$ mutations block the subsequent activation of apoptosis by Atm. In this manner, the Mre $11^{A T L D 1 / A T L D 1}$ mutation creates a scenario similar to the $\mathrm{Atm}^{-/-}$nervous system whereby defective ATM signaling inhibits apoptosis of DNA-damaged neural cells (Lee et al., 2001). Therefore our data provide a mechanistic explanation for the similar neuropathology between A-T and ATLD. We have previously suggested that the loss of ATM prevents apoptosis, leading to incorporation of cells harboring genomic damage into the mature nervous system, and over time these cells presumably succumb to this damage and die (Herzog et al., 1998; Lee et al., 2001). Thus, attenuation of DNA damage-dependent apoptosis in ATLD would mimic A-T, resulting in neurodegeneration characteristic of each disease.

Conversely, in NBS, DNA damage accumulates due to dysfunctional MRN, but Nbs1 mutations do not block Atm-dependent apoptosis and therefore we predict that increased apoptosis occurs during development contributing to microcephaly. This is consistent with defective NBS1 leading to increased genotoxic stress, cancer and radiosensitivity (Kobayashi et al., 2004). As opposed to the $N b s 1^{\triangle B / \triangle B}$ mice, complete disruption of Nbs1 in the murine nervous system leads to microcephaly from DNA damage-induced apoptosis and decreased proliferation (Frappart et al., 2005). In contrast 
to the microcephaly observed in LIG4 syndrome and modeled in the Lig $4^{\text {Nes-cre }}$ mice, neuropathology associated with NBS could be due to damage accumulation in either post-mitotic or proliferating cells as NBS1 is also important during HR (Limbo et al., 2007; Sartori et al., 2007; Tauchi et al., 2002). The $N b s 1^{\Delta B}$ allele results from deletion of exons 4 and 5, producing a protein that lacks the N-terminal FHA and BRCT domains encoded within exons 1 through 5 . In contrast to the $N b s 1^{\Delta B / \Delta B}$ mouse, other models that lack a C-terminus ( $N b s 1^{\Delta C / \Delta C}$ and $N b s 1^{t r 735}$ ), showed reduced apoptosis in some tissues consistent with a defect in ATM activation (Difilippantonio et al., 2007; Stracker et al., 2007). Additionally, deletion of the Nbs1 C-terminus in the context of the $\mathrm{Nbs} 1^{\Delta \mathrm{B}}$ protein (the $N b s 1^{\triangle B C}$ allele) results in apoptotic defects in thymocytes but does not rescue gestational lethality associated with Lig4 loss (data not shown) which is linked to neuraxis-wide apoptosis, suggesting that modulation of apoptosis by Nbs1 is tissuespecific.

As NBS individuals harbor mutations in the FHA and BRCT domains, not the Cterminus, the pathology associated with NBS involves an intact C-terminus. Preservation of the C-terminus presumably allows interaction of the resulting NBS1 protein with ATM and Mre11, and the activation of apoptosis. Although the level of Nbs1 is reduced in Mre $11^{A T L D 1 / A T L D 1}$ cells, there is still a relatively higher level than that of the NBS1 p80 protein found in $N b s 1^{\Delta B / \Delta B}$ tissue. This suggests that the apoptotic defect we observe in the Mre $11^{A T L D 1 / A T L D 1}$ nervous system directly involves the Mre11 mutant protein rather than an indirect effect upon Nbs1. Given the multiple contacts ATM makes with the MRN complex, it is likely that ATLD mutations directly and selectively impact ATMMRN interactions to preclude effective ATM activation (Lee \& Paull, 2004).

Why then do A-T and NBS have many phenotypic similarities outside of the nervous system? These similarities encompass immunodeficiency, cancer and radiosensitivity, and reflect the interrelated roles of MRN and ATM during DNA rearrangements in the immune system and maintenance of genomic integrity in proliferating cells. For example, ATM and NBS1 are required to ensure the fidelity of programmed rearrangements and elimination of cells with inappropriate genomic rearrangements in the immune system (Bredemeyer et al., 2006; Callen et al., 2007; Jankovic et al., 2007; Kracker et al., 2005; Reina-San-Martin et al., 2005). Additionally, both ATM and NBS1 can function in stem and progenitor populations in the hematopoietic and germ cell compartments (Ito et al., 2004; Kang et al., 2002; Takubo et al., 2008). These requirements for ATM and NBS1 contrast the situation in the nervous system where ATM fulfills an indispensable role in DNA damage-induced apoptosis in postmitotic neurons, but not in proliferating neural progenitors where ATR or DNA-PK may be the more important kinases (Lee et al., 2001; Orii et al., 2006).

In summary, our data demonstrate that the respective MRN mutations that lead to NBS and ATLD impact differently upon ATM signaling. These data illuminate the mechanistic underpinnings of the different neuropathology present in ATLD and NBS, suggesting they result from alterations in the modulation of ATM-dependent apoptosis after DNA damage (Shull et al., 2009). 


\section{CHAPTER 3 ATR IS REQUIRED FOR GENOMIC INTEGRITY AND PROLIFERATION OF NEURAL PROGENITORS}

\subsection{Introduction}

The three major phosphoinositol 3-kinase (PI3K)-related protein kinases (PIKKs) involved in signal transduction of the DNA damage response are ataxia-telangiectasia mutated (ATM), ATM and RAD3-related (ATR), and DNA-Protein Kinase catalytic subunit (DNA-PK $\mathrm{C}_{\mathrm{cs}}$ ). ATM and ATR are highly similar in structure and share many overlapping substrates. Both kinases have a preference for phosphorylating serine or threonine residues that are followed by a glycine (Cimprich \& Cortez, 2008). Recent proteomic analysis identified about 700 substrates phosphorylated on ser-gln and thr-gln sites recognized by ATM and ATR, in response to DNA damage. A few examples of such substrates include FANCD2, MDC1, 53BP1, and Orc3 (Matsuoka et al., 2007). ATR has also been reported to phosphorylate both Chk1 and Chk2 (a major ATM substrate) in response to cisplatin (Pabla et al., 2008). Additionally, both ATR and ATM reportedly phosphorylate p53 as well (Banin et al., 1998; Khanna et al., 1998; Tibbetts et al., 1999). Despite these similarities, ATM and ATR are functionally distinct, as evident by early embryonic lethality of $\mathrm{Atr}^{--}$mice versus the viability of $\mathrm{Atm}^{-/-}$mice (Barlow et al., 1996a; Brown and Baltimore, 2000; Brown et al., 1997; Elson et al., 1996b). Inactivating ATM mutations cause the syndrome ataxia-telangiectasia, which is characterized by neurodegeneration, cancer predisposition, immunodeficiency, radiosensitivity, and ocular telangiectasia (Gilad et al., 1996; Savitsky et al., 1995). Hypomorphic mutations in ATR have been identified in a few individuals with Seckel syndrome, which is an autosomal recessive condition characterized by low birth weight, micrognathia, beak-like protrusion of nose, and microcephaly. Mental retardation has been reported in some cases of Seckel syndrome (O'Driscoll et al., 2003). The distinctions between the physiologic requirements of ATM and ATR suggest that though these genes may serve some overlapping roles in DNA damage signaling, each likely fulfills an individual obligation largely dictated by cell type, proliferation status, and the form of damage incurred.

It has been shown in $\mathrm{Atm}^{-/}$mice that newly post-mitotic cells of the developing brain require Atm for apoptotic induction following IR-induced damage. Lig4-deficiency results in Atm-dependant apoptosis of post-mitotic neuron populations during embryogenesis, whereas $X r c c 2^{-/-}$embryos exhibit Atm-independent apoptosis localized to proliferating cells in the developing brain (Orii et al., 2006). This difference in Atm requirement during NHEJ versus during homologous recombination and its non-essential status during embryogenesis suggest that Atm is not the primary signaling molecule adapted to the DDR in proliferating neural precursors. Atr is required for embryonic gastrulation and is involved in maintaining integrity of proliferating tissues in adult mice, with little or no obvious requirement in largely post-mitotic organs such as the mature brain (Brown \& Baltimore, 2000; Ruzankina et al., 2007). While ATM is typically 
described as responding to double strand breaks, ATR activity is associated with multiple forms of damage including replication stress-induced DNA damage, UV-induced pyrimidine dimmers, and base adducts (Cimprich \& Cortez, 2008; Mordes et al., 2008). However, phosphorylation of H2AX on ser 139 is a key characteristic of the DSB response, which can occur as a result of DNA damage from replication fork collapse. Recent in vitro studies have shown that replication fork collapse in the absence of ATR results in H2AX phosphorylation by ATM and DNA-PKcs, and that the accumulation of Rad51 foci (evidence of homologous recombination) is largely dependant upon H2AX (Chanoux et al., 2008). These results indicate that ATR-dependent replication fork stabilization and ATM/DNA-PKcs-mediated H2AX phosphorylation cooperatively function in response to DSBs generated by replication fork collapse. Thus, despite distinct physiologic requirements and separate modes of activation, ATM and ATR likely act synergistically to ensure genomic integrity during all stages of life.

Replication protein A (RPA)-coated stretches of ssDNA are formed during replication, and are important for localizing ATR to damage sites (Costanzo et al., 2003; Zou \& Elledge, 2003). ATR-interacting protein (ATRIP) is an essential co-factor for ATR and is required for targeting ATR to RPA-coated ssDNA, to which it directly binds (Ball et al., 2007; Cortez et al., 2001). Additionally, topoisomerase (DNA) II binding protein 1 (TopBP1), is involved in activation of the ATR-ATRIP complex through interactions with both ATRIP and ATR (Kumagai et al., 2006; Mordes et al., 2008). ATR signaling further depends on the RAD9-RAD1-HUS1 (911) complex that recognizes a DNA end adjacent to RPA bound ssDNA (Bermudez et al., 2003; Ellison \& Stillman, 2003; Zou et al., 2003). ATM activation occurs through a different series of signaling events involving autophosphorylation and dependence upon the MRN complex (Bakkenist \& Kastan, 2003; Cerosaletti \& Concannon, 2004; Cerosaletti et al., 2006; Kozlov et al., 2006; Lee \& Paull, 2004; Lee \& Paull, 2005). Figure 3.1 depicts simple models for the separate activation mechanisms and consequent signaling pathways of ATM and ATR (Cimprich \& Cortez, 2008) In vitro studies using Xenopus egg extracts and various mammalian cell lines have implicated MRN-dependent ATM activity as an ATR activator through phosphorylation of TopBP1 in a cell cycle specific manner (Adams et al., 2006; Cuadrado et al., 2006; Jazayeri et al., 2006; Myers \& Cortez, 2006; Yoo et al., 2007). Alternatively, it has been reported that ATR phosphorylates and activates ATM in response to UV and replication fork stalling in ATR-Seckel fibroblasts (Stiff et al., 2006). Thus, while ATR and ATM are thought to have predominantly distinct signaling mechanisms, there is also evidence of interacting mechanisms in addition to overlapping substrates between the two kinases. Nonetheless, the interrelationship between ATR and ATM is complex, and many aspects remain to be elucidated. Deciphering the relevance of in vitro data to in vivo tissue-specificity of ATR function remains a challenge as well.

To determine what relationship exists between ATR and ATM signaling in the nervous system, we used a conditional knock-out strain to inactivate Atr in the nervous system by breeding it onto a Nestin-cre transgenic line (Brown \& Baltimore, 2000). Atr ${ }^{\text {loxPlloxP }}$; Nestin-cre animals were born at a Mendelian frequency, but showed post-natal 

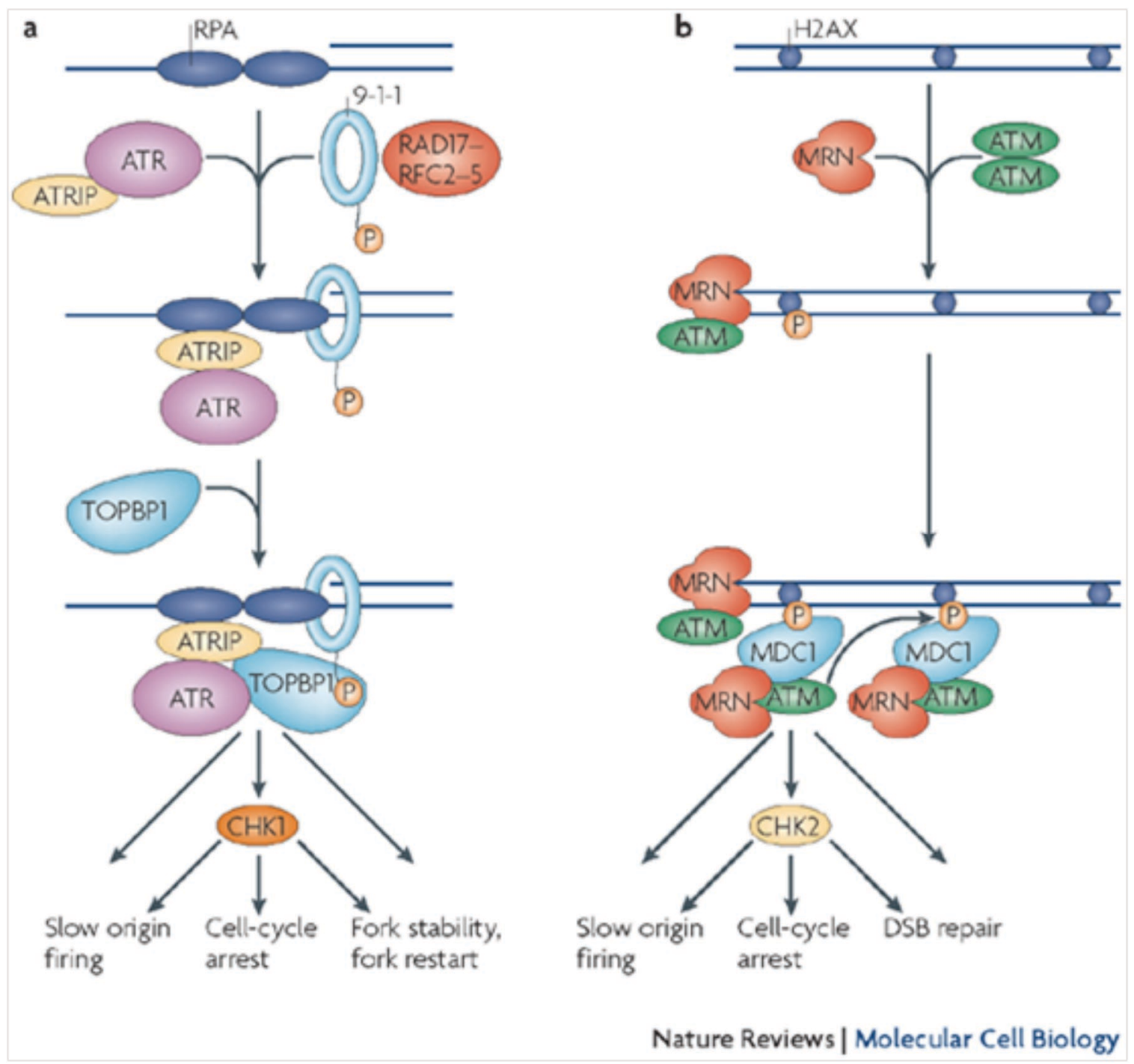

Figure 3.1 Activation and signaling pathways of ATR and ATM.

(a) The ATR-ATRIP and the 911 complexes are recruited to RPA-coated ssDNA. ATRIP binds RPA and the 911 clamp is loaded onto the 5' primer junction with the aid of the RAD17-RFC2-5 complex. TopBP1 binds and activates ATR in an ATRIP-dependent manner. ATR is then able to phosphorylate Chk1 and other substrates to mediate origin firing, cell-cycle arrest, and replication fork stability. (b) DSB formation leads to H2AX phosphorylation and recruitment of the MRN complex and ATM to the break site. MRN activity promotes dissociation of the inactive ATM multimer into its monomeric form through autophosphorylation events. ATM binds to the MRN complex the Nbs1 Cterminus. Binding of MDC1 to $\gamma-\mathrm{H} 2 \mathrm{AX}$ results in recruitment of additional ATM-MRN complexes to the damage site. ATM activity mediates the DSB response through phosphorylation of substrates such as Chk2. This figure was reprinted by permission from Macmillan Publisher Ltd: Nature Reviews Molecular Cell Biology Volume 9 No. 8 (Cimprich \& Cortez, 2008), copyright 2008, page 617. 
lethality and neurogenesis defects associated with increased apoptosis and reduced proliferation of neural progenitor cells. Because the cerebellum was most dramatically affected and is of particular interest in comparing the requirement of ATM with that of ATR in the nervous system, most emphasis in this study was placed on that region. The cerebellum arises from the isthmus, which forms the metencephalic-mesencephalic junction of the neural tube (Hallonet \& Le Douarin, 1993; Martinez et al., 1991). Lineage analysis using chick-quail chimeras revealed contribution of both the mesencephalic and metencephalic plates to formation of the cerebellar anlage (Hallonet \& Le Douarin, 1993). Cerebellar cells originate in the ventricular zone (VZ) and the rhombic lip. The ventricular zone lining the dorsal region of the fourth ventricle (V4) produces Purkinje cells (PCs) and interneurons. The rhombic lip arises between the neural tube and the roof plate of V4; it gives rise to granule cell precursors that migrate over the cerebellar surface to form the EGL. Cerebellar development is not complete until months after birth in humans or about P21 in mice (Carletti \& Rossi, 2008; Goldowitz \& Hamre, 1998; Sillitoe \& Joyner, 2007; Wang \& Zoghbi, 2001; Wingate, 2001). PCs exit the cell cycle and migrate into the cerebellar anlage between E11 and E13 in mice (Edwards et al., 1990; Miale \& Sidman, 1961; Morales \& Hatten, 2006). Purkinje cells mediate replication of the granule cell progenitors in the EGL, as transgenic mice in which Purkinje cells are ablated at specific developmental times showed reduced mitotic activation of EGL cells in regions corresponding to PC loss (Smeyne et al., 1995). PCs produce Sonic hedgehog, which regulates granule cell expansion through its receptor Patched (Goodrich et al., 1997; Traiffort et al., 1998). Organization of the PCs into the mature monolayer depends upon proper formation and function of granule cells. This is apparent in Math1-null mice, in which improper granule cell generation leads to abnormal development of the PC layer (Ben-Arie et al., 1997). Rhombic lip cells are committed to becoming proliferative EGL neurons, and undergo final maturation and migration to form the inner granule layer (IGL) between birth and about P21, by which time proliferation is complete and the EGL has disappeared (Sillitoe \& Joyner, 2007; Wang \& Zoghbi, 2001). Figure 3.2 demonstrates gross development of the murine cerebellum from E11 to adulthood (Goldowitz \& Hamre, 1998). Figure 3.3 depicts post-natal development of the cerebellum, during which granule cells migrate through the Purkinje cell layer (PCL) to form the mature internal granule cell layer, and the dendritic arborizations fully maturate and extend their branches to the climbing fibers of the molecular layer (Goldowitz \& Hamre, 1998).

Despite numerous in vitro studies implicating a direct relationship between ATM and ATR signaling, our genetic analysis of Atm and Atr requirements indicate that these genes appear to function independently of each other in the CNS. We found that Atr is required in proliferating cells while as previously reported Atm is critical in post-mitotic neurons (Herzog et al., 1998; Lee et al., 2001; Ruzankina et al., 2007). Furthermore, we show that Atr loss induces p53-dependant apoptosis in proliferating neuroprogenitors and p53-independent cell cycle arrest that results in severe cerebellar dysgenesis, olfactory bulb malformation, and microcephaly, as well as growth delay and post-natal lethality. 


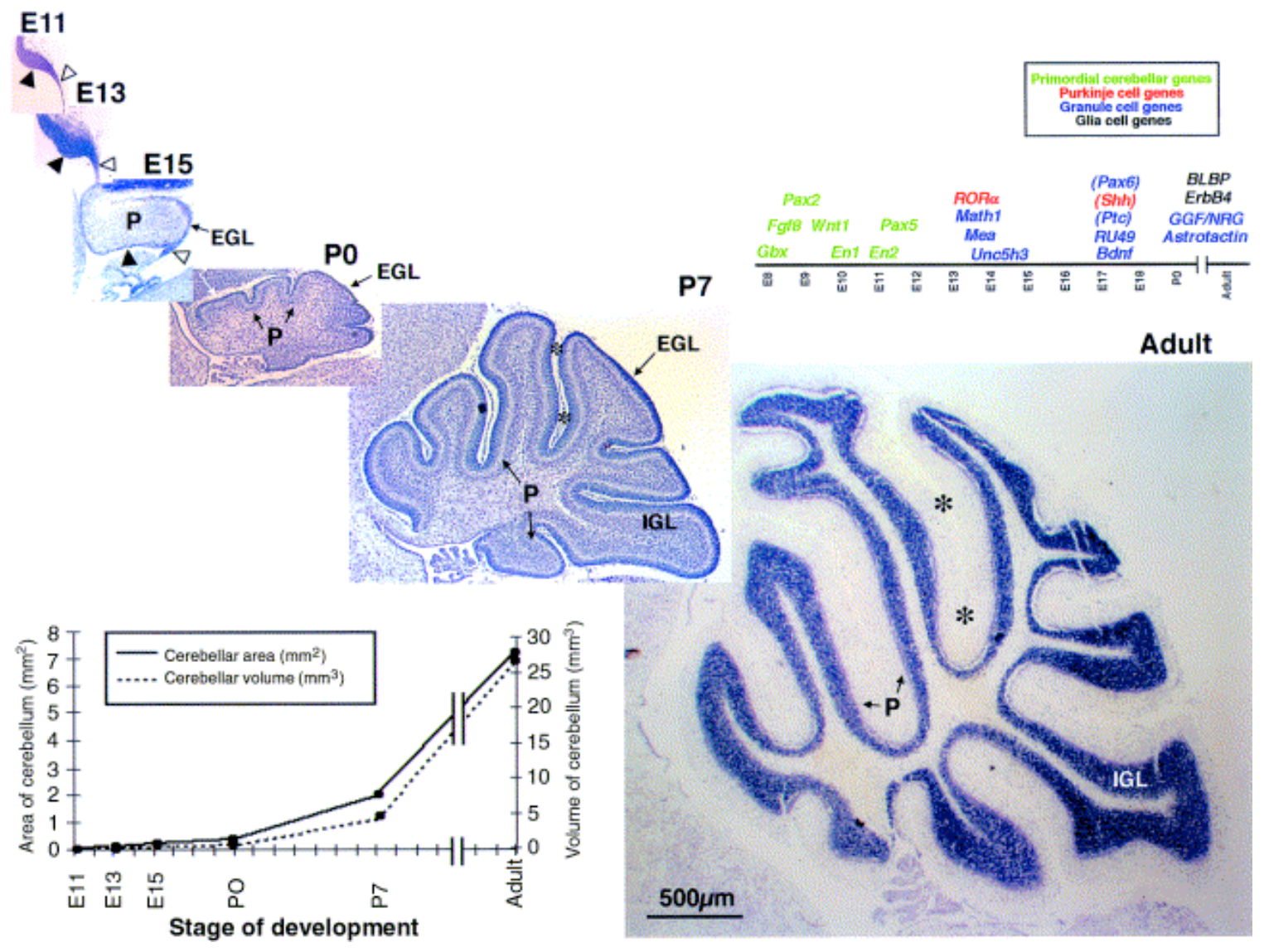

Figure 3.2 Dramatic changes in cerebellar morphology that occur between embryogenesis and adulthood.

Sagittal sections of the mouse cerebellum at E11, E13, E15, P0, P7, and adulthood show the dramatic increase in area and volume (bottom left graph) that occurs between early specification of the cerebellar anlage and maturation. Filled arrowheads indicate the neuroepithelium lining the dorsal wall of the fourth ventricle. Open arrowheads indicate the rhombic lip. Asterisks indicate the primary fissure. The timeline in the upper right corner illustrates some of the genes that are required for normal cerebellar development and the ages at which mutations in these genes manifest pathologically. Genes that are critical to early stages of development are in green. Genes specific to granule cells are denoted in blue, those specific to Purkinje cells are in red, and those associated with radial glia are in black. Abbreviations: external granular layer (EGL), Purkinje cell layer (P), internal granule cell layer (IGL). Reprinted from Trends in Neuroscience, Vol. 21, No. 9, Dan Goldowitz and Kristin Hamre, The cells and molecules that make a cerebellum, 375-382, 1998, with permission from Elsevier. 


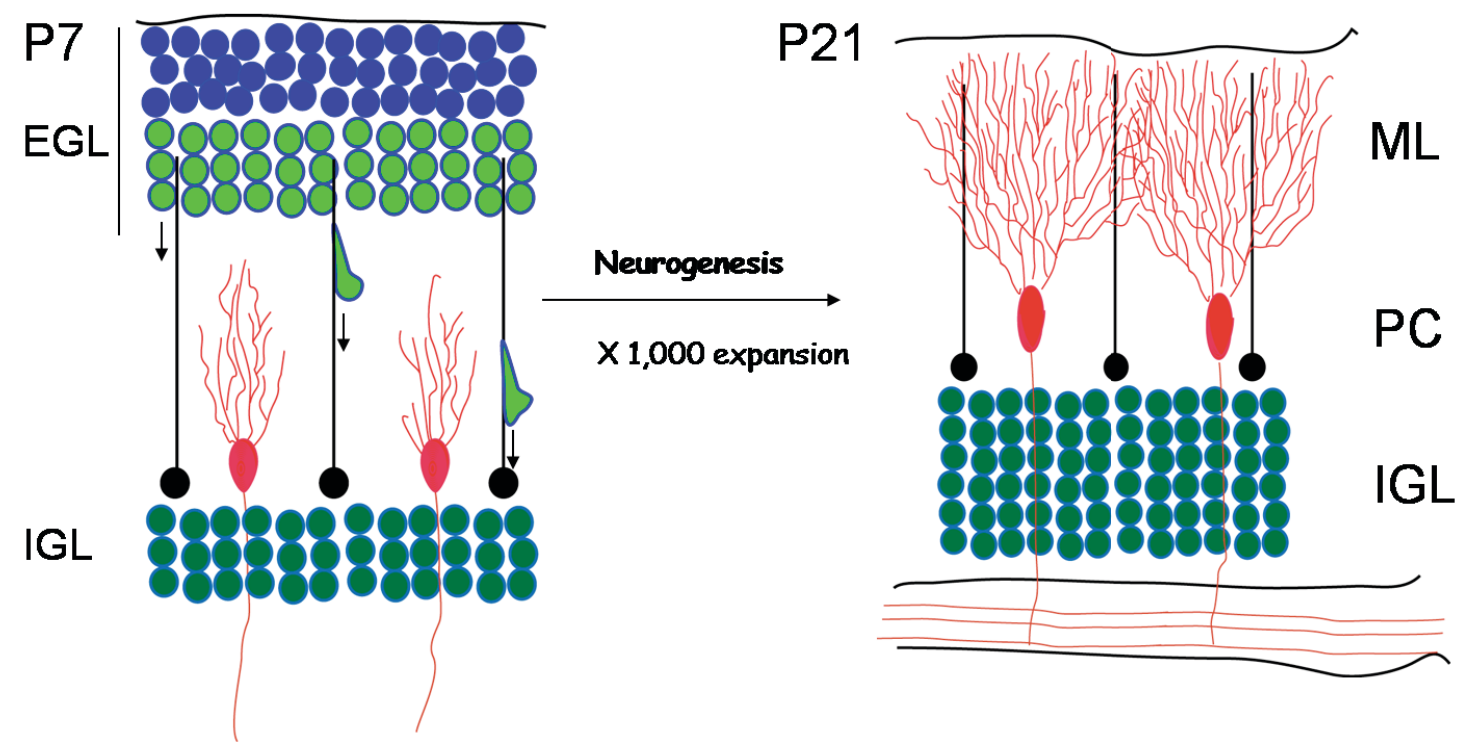

Figure 3.3 Depiction of post-natal cerebellar development.

At birth, the external granule cell layer is comprised of an outer layer of proliferating granule cell precursors (blue) and inner layer of newly post-mitotic, differentiating granule cells (green). Upon exiting the cell cycle, differentiating granule cells migrate along radial glia fibers (black), through the Purkinje cell layer (red) and into the internal granule cell layer, where they fully differentiate into mature granule cells (dark green). Between P7 and P21, the EGL has disappeared and the dendritic arborizations of the Purkinje cells (PC) have matured into elaborate projections that connect with the climbing fibers of the molecular layer (ML). Expansion of the granule cells during postnatal development results in an approximately 1000 fold increase in total cerebellar size between birth and P21 (Goldowitz \& Hamre, 1998). Figure is courtesy of Dr. Peter McKinnon, February 10, 2009. 


\subsection{Experimental Procedures}

\subsubsection{Animals}

Atr ${ }^{\text {loxPlloxP }}$ were obtained from Dr. Eric J. Brown and crossed onto a Nestin-cre transgenic line to achieve deletion throughout the nervous system.

To generate a conditional Atm allele, Dr. Peter McKinnon obtained a murine BAC genomic clone (strain, 129Ola) containing the Atm genomic locus, into which he inserted a LoxP sequence into a unique BamHI site upstream of the targeted exon and a floxed NeoTK construct cloned into a XbaI site directly downstream of exon 58 to generate $\mathrm{p}$ Atm $^{l o x P}$. ES cells (W9.5; 129S1/SvImJ) were electroporated with linearized pAtm ${ }^{\text {LoxP }}$ and selected with G418 antibiotic. Correctly targeted cells were identified by southern blot analysis (Dr. Helen Russell). Targeted ES clones were then electroporated with pMC-cre, followed by selection in FIAU (5-iodo-2'-fluoro-2'deoxy-1arabinofuranosyluracil) to kill cells containing TK. ES cells were then injected into blastocysts and the resultant chimeric mice assessed for germline transmission of the floxed Atm allele (St. Jude Children's Research Hospital Transgenic Core). Nestin-cre mice (B6.Cg-Tg(Nes-cre) $1 \mathrm{Kln} / \mathrm{J}$; JAX \#003771) were used to delete Atm throughout the nervous system.

Animals were housed in an Association for the Assessment and Accreditation of Laboratory Animal Care (AAALAC)-accredited facility and were maintained in accordance with the NIH Guide for the Care and Use of Laboratory Animals. The St. Jude Children's Research Hospital IACUC approved all procedures for animal use.

\subsubsection{Real-time PCR}

Genomic DNA was phenol/chloroform extracted from frozen sections and suspended in TE buffer. DNA concentration was determined from the $\mathrm{A}_{260}$ as measured using an Ultrospec 2000 UV/Visible Spectrophotometer (Pharmacia Biotech). Standard DNA was serially diluted at 1:5 from a starting concentration of $100 \mathrm{ng} / \mu \mathrm{L}$. Unknown samples were diluted to $100 \mathrm{ng} / \mu \mathrm{L}$, such that each reaction contained an equal amount of DNA. $\beta$ DNA was added to $\mathrm{iQ}^{\mathrm{TM}}$ SYBR ${ }^{\circledR}$ Green supermix (BioRad) containing forward and reverse primers for either the targeted exon 44 of Atr $/ 58$ of Atm or the control exon 29 of Atr/52 of Atm. PCR reaction was carried out using the iQ ${ }^{\mathrm{TM}} 5$ Multicolor Real-Time PCR Detection System (BioRad). Measurement numbers were automatically assigned based on SYBR green amounts fit to a standard curve. The amount of the targeted exon product was normalized to that of the control exon for each sample.

Atr exon 29 forward primer: 5' ACTCTGGCTGTAGCGTCCTTTC Atr exon 29 reverse primer: 5' TGCTTCTTTTCTGTAATAAATGACTCAAA Atr exon 44 forward primer: 5' GAAAGGAGCTTCGCCAGTGT 
Atr exon 44 reverse primer: 5' GGGCAGGAGTAATTCTTGGAATAC Atm exon 52 forward primer: 5' ATGGAATGAAGATTTCATCCTATAAGTTT

Atm exon 52 reverse primer: 5' ATCCTAGGCCTCCCGTCATTT

Atm exon 58 forward primer: 5' TCAGCGAAGCGGTGTTCTC

Atm exon 58 reverse primer: 5' TCATTTGGCCTGTATCTTCTATGTG

\subsubsection{Histology and immunohistochemistry}

Pregnant female mice were euthanized through cervical dislocation and embryos were drop-fixed in 4\% PFA either overnight or 2-3 days depending upon age. Post-natal day 5 mice were sacrificed via transcardial perfusion using 4\% PFA. Tissues were further fixed in 4\% PFA overnight, and either cryoprotected in 25\% PBS-buffered sucrose solution and embedded in O.C.T. (Tissue-Tek). Frozen tissue was sectioned at $10 \mu \mathrm{m}$ thickness using a Microm HM500 M cryostat.

Antigen retrieval was used for all immunohistochemistry using the anti-phospho$\gamma \mathrm{H} 2 \mathrm{AX}$ (1:50; Cell Signaling), anti-53BP1 polyclonal (1:500; Bethyl laboratories), antip53 (CM5; 1:500; Vector Laboratories), anti-p53 phospho-Ser-15 (1:500; Cell

Signaling), anti-active caspase 3 (1:500; BD Biosciences), anti-PCNA (1:500; Santa Cruz) and anti-Ki67 (1:1000; Vector Laboratories) antibodies. Antigen retrieval was not necessary for labeling with anti-Sox2 (1:1000; Chemicon) or anti-ssDNA (1:300; Chemicon). Immunostaining against anti- $\beta$ tubulin III TUJ1 (1:500; Babco) was equally efficient with or without antigen retrieval. For immunofluorescent staining, sections were subsequently immunolabelled with Cy3-conjugated goat anti-mouse (1:400; Jackson Immunoresearch) or FITC-conjugated goat anti-rabbit secondary antibodies (1:500; Jackson Immunoresearch) and mounted with Vectashield anti-fade reagent containing DAPI (Vectorlabs).

Immunohistochemistry for activated caspase-3 or p53-ser15 (murine homologue is serine 18) was performed overnight at room temperature after quenching endogenous peroxidase activity with $0.6 \%$ hydrogen peroxide in methanol. Immunoreactivity was visualized with a vasoactive intestinal peptide substrate kit (V.I.P.; Vector Labs) according to the manufacturer's directions after tissues were treated with biotinylated secondary antibody and avidin DH-biotinylated horseradish peroxidase-H complex (Vectastain Elite kit; Vector Labs). Sections were counterstained with $0.1 \%$ methyl green (Vector Labs), dehydrated, and mounted in DPX reagent. Nissl staining was performed after cryosections were allowed to dry at room temperature and was followed by dehydration in alcohol and xylene. Apoptotic cells were detected with ApopTag Fluorescein in situ apoptosis detection kit (Chemicon international) according to the manufacturer's instructions. Quantitation of TUNEL, caspase-3, p53-ser15, ssDNA, or SOX2 positive cells was determined by counting positive signal from images equivalent in size and magnification between genotypes. Images were captured using an Axioskop 2.0 microscope (Carl Zeiss) and a SPOT camera (Diagnostic Instruments, Inc). 


\subsection{Results}

\subsubsection{Atr deficiency results in microcephaly and cerebellar dysgenesis}

In order to evaluate the specific role of ATR during neurogenesis, Atr ${ }^{\text {loxP/loxP }}$ mice were crossed onto the same Nestin-cre transgenic line used with the Lig $4^{\text {loxP/loxP }}$ mice reported in Chapter 2. Atr ${ }^{+/ l o x P}$; Nestin-cre mice were healthy and identical to $\mathrm{Atr}^{+/+}$; Nestin-cre counterparts. Thus either $\mathrm{Atr}^{+/+}$; Nestin-cre or $\mathrm{Atr}^{+/ l o x P}$; Nestin-cre genotypes were used interchangeably as controls and will be referred to collectively as Atr ${ }^{C t r l}$ for simplicity. Atr ${ }^{\text {loxP } / l o x P}$ mice were also compared with $\mathrm{Atr}^{+/+}$; Nestin-cre or $\mathrm{Atr}^{+/ l o x P}$; Nestin-cre animals. Nestin-cre status had no noticeable effect upon $\mathrm{Atr}^{+/+}$mice, in contrast to the report that Nestin-Cre expression can cause neurogenesis defects when highly expressed in neural progenitor cells (Forni et al., 2006). When Nestin-cre positive controls were not available, Atr $^{+/+}$, Atr $^{+/ l o x P}$, or Atr $^{\text {loxP } / l o x P}$ animals were used as controls as well. Preference was always given to mice containing the Nestin-cre transgenes, when available. Real-time PCR was used to gauge efficiency of excision of the floxed exon 44 in E13.5 $\mathrm{Atr}^{\text {Nes-cre }}$ CNS compared to $\mathrm{Atr}^{+/+}$; Nestin-cre littermates. DNA extracted from the whole CNS of 3 separate $A t r^{\text {Nes-cre }}$ embryos showed $60-80 \%$ deletion efficiency (Figure 3.4). In situ hybridization is currently underway to specifically determine which areas of the CNS do and do not express an Atr mRNA transcript.

Atr $^{\text {Nes-cre }}$ animals were born at a frequency consistent with expected Mendelian ratios (data not shown), but died during early post-natal life. Typically Atr ${ }^{\text {Nes-cre }}$ animals did not survive beyond P7, however there were occasional animals that survived up to P10 or P11. (Two such mice were examined histologically and found to have a less severe phenotype than that of the typical Atr ${ }^{\text {Nes-cre }}$ animals described below. Presumably such older animals were expressing the Nestin-cre transgene at a lower than usual level.) Cause of death remains undetermined, but is suspected to be associated with malnutrition, possibly due to inability to efficiently swallow and retain milk.

Atr ${ }^{\text {Nes-cre }}$ pups showed severe overall growth deficiency and had consistently smaller brains compared to littermate controls (Figure 3.5A-B). We attribute the small body size of $A t r^{\text {Nes-cre }}$ animals, at least partially, to malnutrition based upon our observation that their stomachs did not contain milk, which was visible in control littermates. To determine if larger littermates were out-competing the Atr $^{\text {Nes-cre }}$ animals, littermates were removed from the cage leaving only the Atr ${ }^{\text {Nes-cre }}$ pups with the mother. This had no effect upon feeding or survival. Attempts were also made to hand feed the animals using canine milk replacement formula. We chose canine formula as the dietary habits of dogs and mice seemed more similar than that of cats and mice; however we do not know if feline formula would have been more suitable. One $\mathrm{mL}$ syringes were used in our attempts to hand feed the Atr $^{\text {Nes-cre }}$ pups. This endeavor was a failure, as the formula was immediately regurgitated. Thus we speculate that dysphagia contributed to malnourishment and ultimate lethality of post-natal Atr $^{\text {Nes-cre }}$ mice. 


\section{Real-time PCR of floxed \\ Atr exon in E13.5 Atr $^{\text {Nes-cre }}$ CNS}

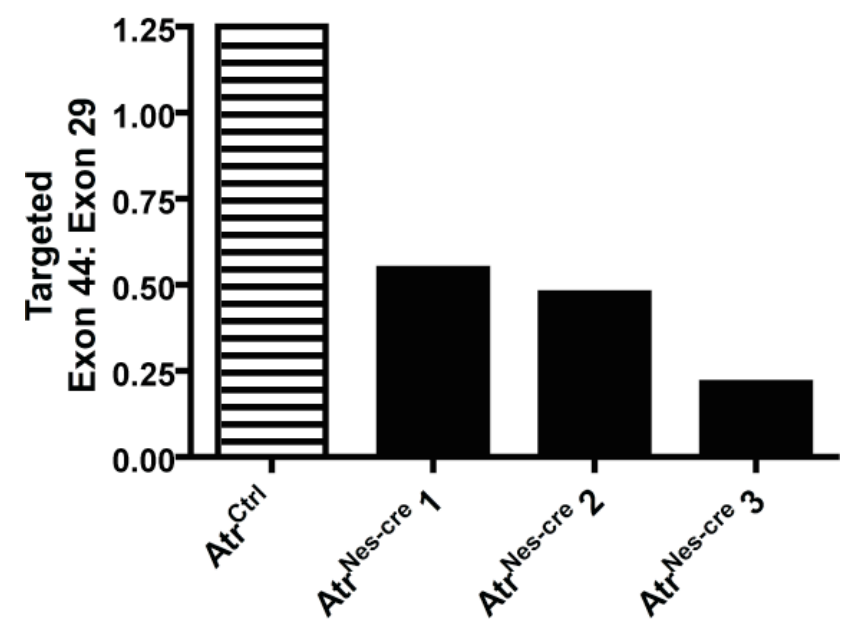

Figure 3.4 Nestin-cre mediated deletion efficiency of floxed Atr region in E13.5 Atr ${ }^{\text {Nes-cre }}$ CNS.

Real-time PCR was used to ascertain deletion efficiency of exon 44 in the Atr ${ }^{\text {Nes-cre }}$ E13.5 CNS. Results for exon 44 were normalized to those for exon 29. 


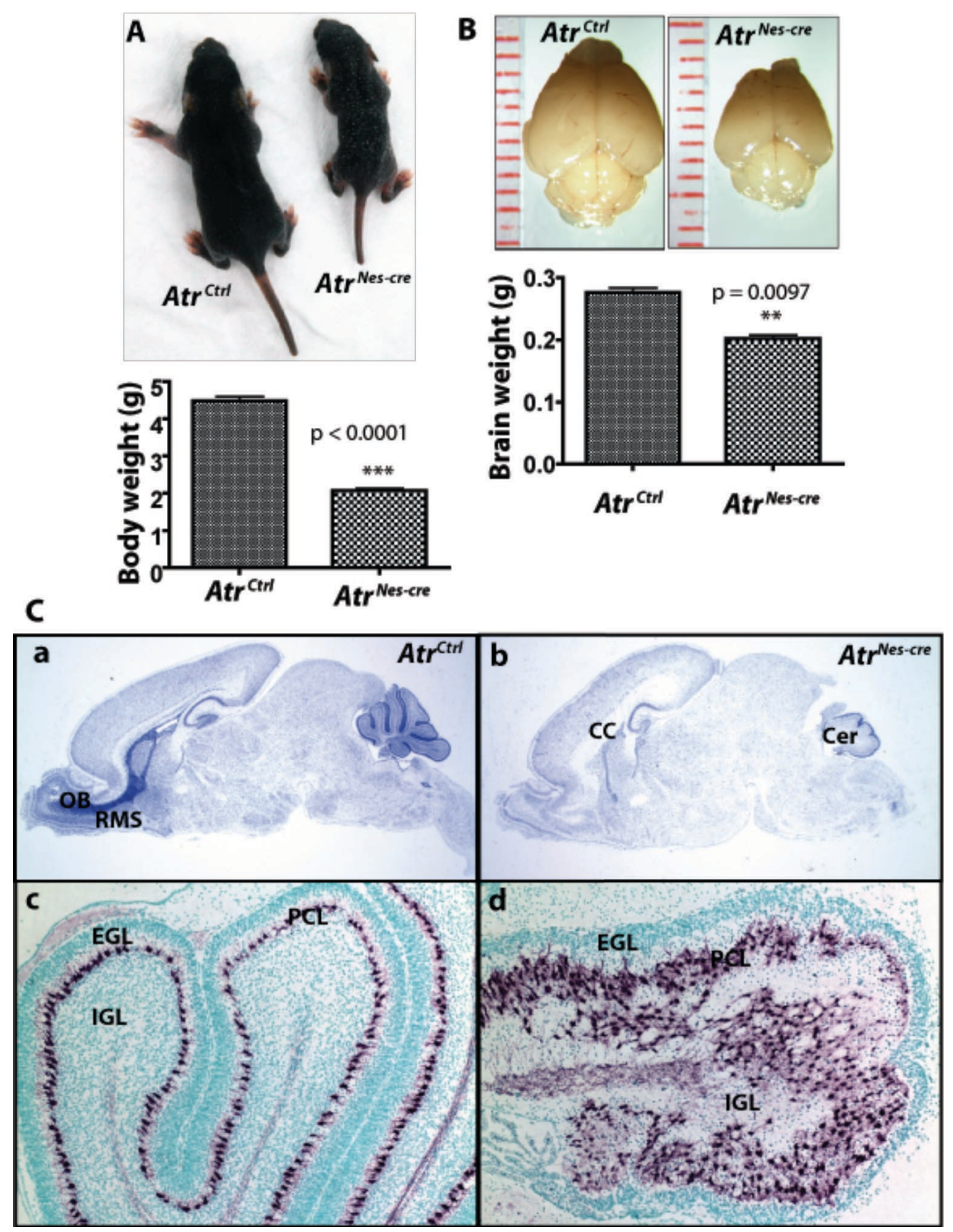

Figure 3.5 $\mathrm{Atr}^{\text {Nes-cre }}$ mice showed severe growth defects accompanied by microcephaly and neurogenesis defects.

(A) P6 Atr ${ }^{C t r l}$ and $A t r^{\text {Nes-cre }}$ littermates show significant size difference, with $A t r^{\text {Nes-cre }}$ animals being $\sim 50 \%$ smaller than controls. (B) $A t r^{N e s-c r e}$ and $A t r^{C t r l}$ brains were collected from P6 littermates. Atr ${ }^{\text {Nes-cre }}$ mice have significantly smaller brains than $A t r{ }^{C t r l}$ mice. (C) Nissl stained sections revealed structural abnormalities in the Atr ${ }^{\text {Nes-cre }}$ olfactory bulb (OB), corpus callosum (CC), rostral migratory stream (RMS), and cerebellum (Cer) in comparison to Atr $^{C t r l}$ (a-b). Immunostaining against calbindin D28K revealed disruption of Purkinje cell layer (PCL) and decreased cellular density of the internal granule layer (IGL) in Atr ${ }^{\text {Nes-cre }}$ animals (c,d). Magnification of Nissl stained panels is $15 x$. Magnification of calbindin-stained sections is 100x. 
The cerebellum was most dramatically affected by Atr loss as manifested by lack of foliation and mislocalization of calbindin-positive Purkinje cells (Figure 3.5C c-d), consistent with defective granule cell generation as described in other mouse models and reminiscent of the phenotype described in the $\mathrm{Nbn}^{f 6 / f 6}$; Nestin-cre mouse in which Nbs1 is conditionally deleted (Ben-Arie et al., 1997; Frappart et al., 2005; Jensen et al., 2002). Nissl staining revealed structural abnormalities in the forebrain of Atr $^{\text {Nes-cre }}$ pups as well. Most notably, decreased cellularity of the corpus callosum was frequently observed and the rostral migratory stream (RMS) seemed largely nonexistent in sagittal sections (Figure 3.5C a-b). The RMS is comprised of progenitor cells that originate in the dorsal wall of the lateral ventricle, commonly referred to as the ventricular zone (VZ), and possibly in the ganglionic eminence (GE), then migrate to the olfactory bulb where they mature into inhibitory interneurons (Corbin et al., 2008; Lledo et al., 2008). Nissl stain was used on coronal sections of P5 $\mathrm{Atr}^{\mathrm{Ctrl}}$ and $\mathrm{Atr}^{\mathrm{Nes}-\mathrm{cre}}$ olfactory bulb to confirm loss of the proliferating cells that comprise the RMS and resultant structural malformation. The olfactory bulb is a layered structure comprised of different cells types. In Atr $^{\text {Nes-cre }}$ pups, the glomerular layer (GL), the external plexiform layer (EPL), and the mitral cell layer (MCL) appeared largely normal though cellularity seemed decreased. However, the internal plexiform layer (IPL) and granule cell layer (GCL) were rather undeveloped (Figure 3.6 upper panels). Consistent with the sparse population of the IPL and GCL, the RMS was nearly non-existent at age P5 in the Atr ${ }^{\text {Nes-cre }}$ animals (Figure 3.6 lower panels). Cellularity appeared drastically decreased throughout the cerebral cortex (CTX) and the corpus callosum (CC) as well (Figure 3.7).

\subsubsection{Atr loss was associated with increased DNA damage and p53- dependent apoptosis in the developing brain}

Beginning at E13.5, DNA damage, as assessed by immunostaining against $\gamma$ H2AX, was apparent in the EGL and GE. This was not a surprising finding, as we predicted damage would be sustained in proliferating cells and lead to cell cycle arrest and/or apoptosis, based upon the report that ATR loss leads to H2AX phosphorylation upon replication stalling in MEFs (Brown \& Baltimore, 2003). The surprising finding

was that $\gamma-\mathrm{H} 2 \mathrm{AX}$ foci were not uniformly present in all proliferating areas of the Atr ${ }^{\text {Nes-cre }}$ brain. At E13.5, the EGL contained a subset of cells that were positive for $\gamma$-H2AX, whereas the RL did not exhibit signs of DNA damage though it is comprised of proliferating cells. Indeed it was not until E16.5 that any $\gamma$-H2AX was detected in the RL of Atr $^{\text {Nes-cre }}$ mice (Figure 3.8).

The GE also exhibited substantial damage by E13.5 based upon immunostaining against $\gamma$-H2AX (data not shown). Between E13.5 and E17.5, the level of damage in the GE increases with a peak around E16.5/E17.5 and a trough at E18.5 (Figure 3.9). The GE provides inhibitory interneurons to the cortex, the olfactory bulb via the RMS, and the striatum. Different subtypes of inhibitory interneurons arise from spatially distinct progenitor cell populations of the ganglionic eminence (Corbin et al., 2008; Wonders \& Anderson, 2005). The medial ganglionic eminence appears to be the main source of somatostatin-positive interneurons and of parvalbumin-positive cortical interneurons. 


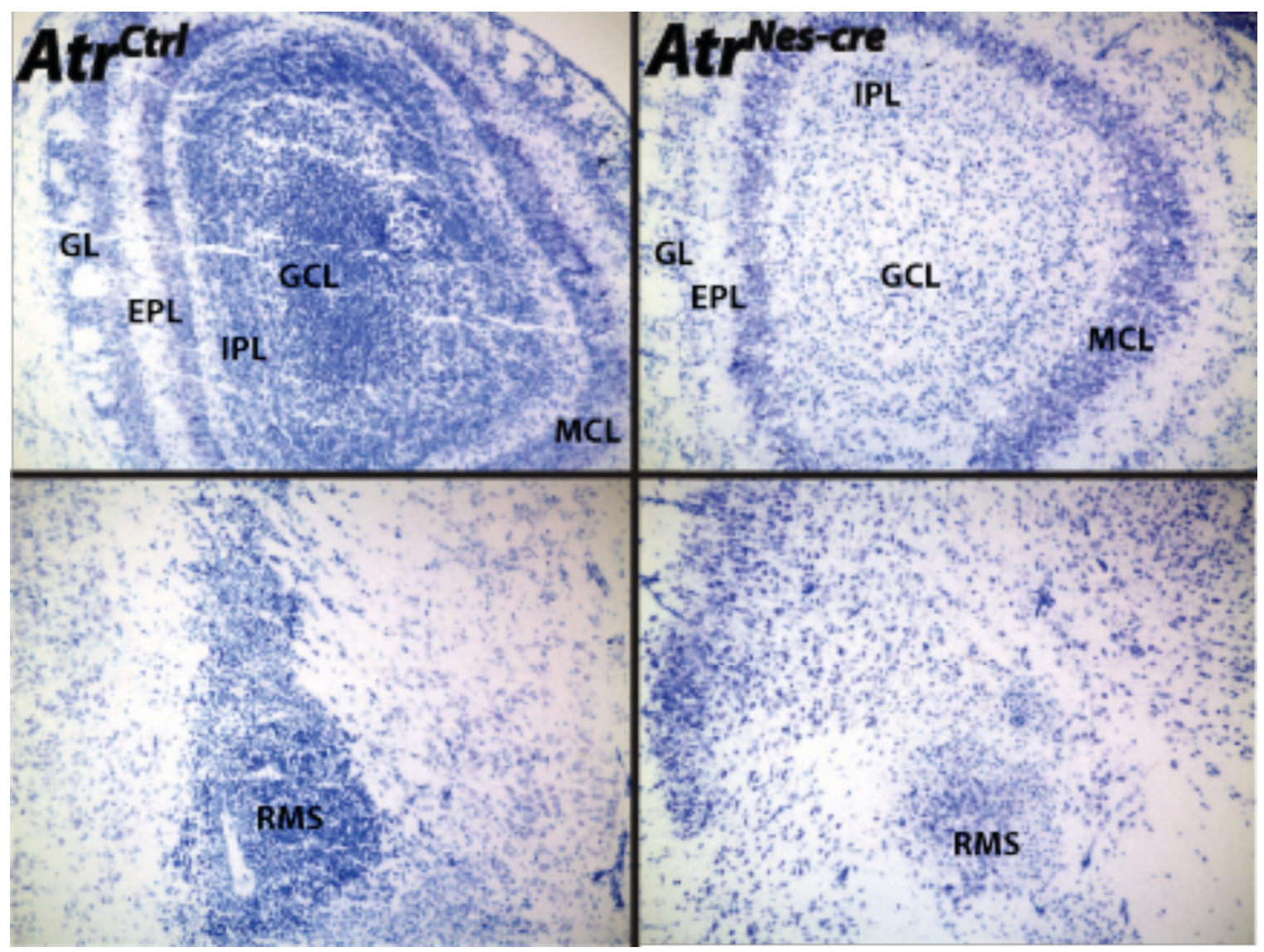

Figure 3.6 Nissl stained coronal sections of the P5 Atr $^{\text {Nes-cre }}$ olfactory bulb and rostral migratory stream.

Coronal sections of the olfactory bulb (upper panels) and the rostro-ventral forebrain (lower panels) revealed severe depletion of the rostral migratory stream (RMS) and decreased cellularity in the internal plexiform layer (IPL) and granule cell layer (GCL) of the Atr $^{\text {Nes-cre }}$ mice (right) in comparison to controls (left). The mitral cell layer (MCL) and the external plexiform layer (EPL) appeared similar to those of the Atr $^{\text {Ctrl }}$ samples though the glomerular layer (GL) seems sparse in Atr $^{\text {Nes-cre }}$ samples. Magnification is 100x. 


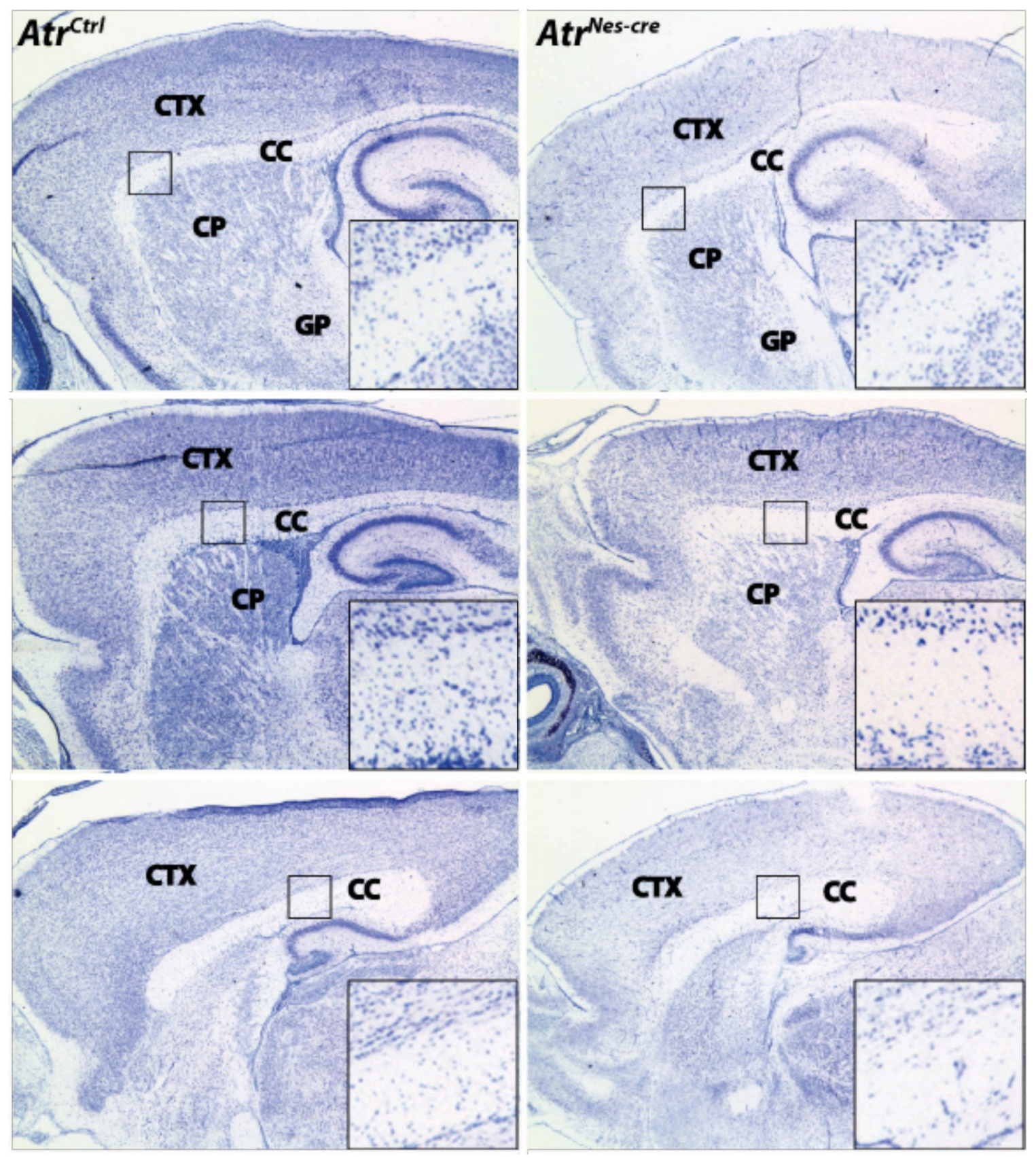

Figure 3.7 P5 Atr ${ }^{\text {Nes-cre }}$ forebrain stained for Nissl substance.

Sagittal sections prepared from P5 Atr ${ }^{C t r l}$ and $A t r^{\text {Nes-cre }}$ brain samples were stained with Nissl. Though cellularity appears decreased in most regions, overall structure is largely preserved in the Atr ${ }^{\text {Nes-cre }}$ samples. The corpus callosum (CC) showed dramatically decreased cellularity. Sections progress lateral to medial from top to bottom. Other abbreviations: cortex (CTX), globus pallidus (GP), caudate putamen (CP). Magnification is $100 x$. 


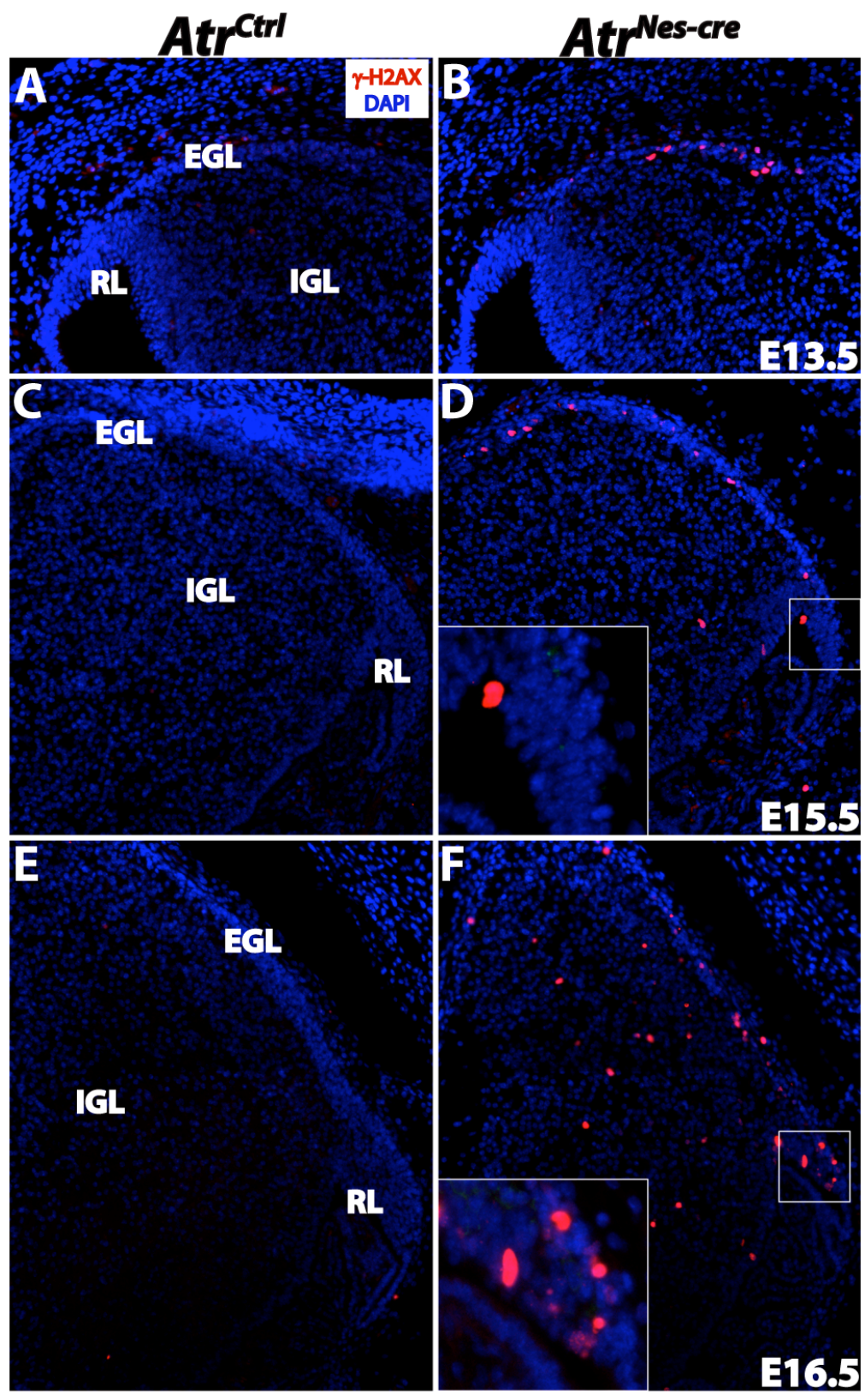

Figure 3.8 Pattern of $\boldsymbol{\gamma}$-H2AX foci in the Atr $^{\text {Nes-cre }}$ cerebellum.

(A-B) E13.5 Atr ${ }^{\text {Nes-cre }}$ samples showed increased immunostaining against $\gamma$-H2AX (red) in the external granule cell layer (EGL) compared to Atr $^{\text {Ctrl }}$ littermates, but not in other regions of the developing cerebellum. (C-D) At E15.5, there was no substantial indication of DNA damage in the rhombic lip (RL) of Atr $^{\text {Nes-cre }}$ samples in comparison to controls, but some damage had arisen in the internal granule cell layer (IGL). (E-F) At E16.5, the RL showed increased $\gamma-\mathrm{H} 2 \mathrm{AX}$ signal and the IGL had sustained more damage than at E15.5 in Atr $^{\text {Nes-cre }}$ samples. Atr ${ }^{\text {Ctrl }}$ samples did not show appreciable $\gamma$-H2AX signal at any stage $(\mathrm{A}, \mathrm{C}, \mathrm{E}) . \mathrm{N}$ is at least 2 for each genotype at each embryonic stage. Magnification is 200x. Nuclei were stained with DAPI (blue). 


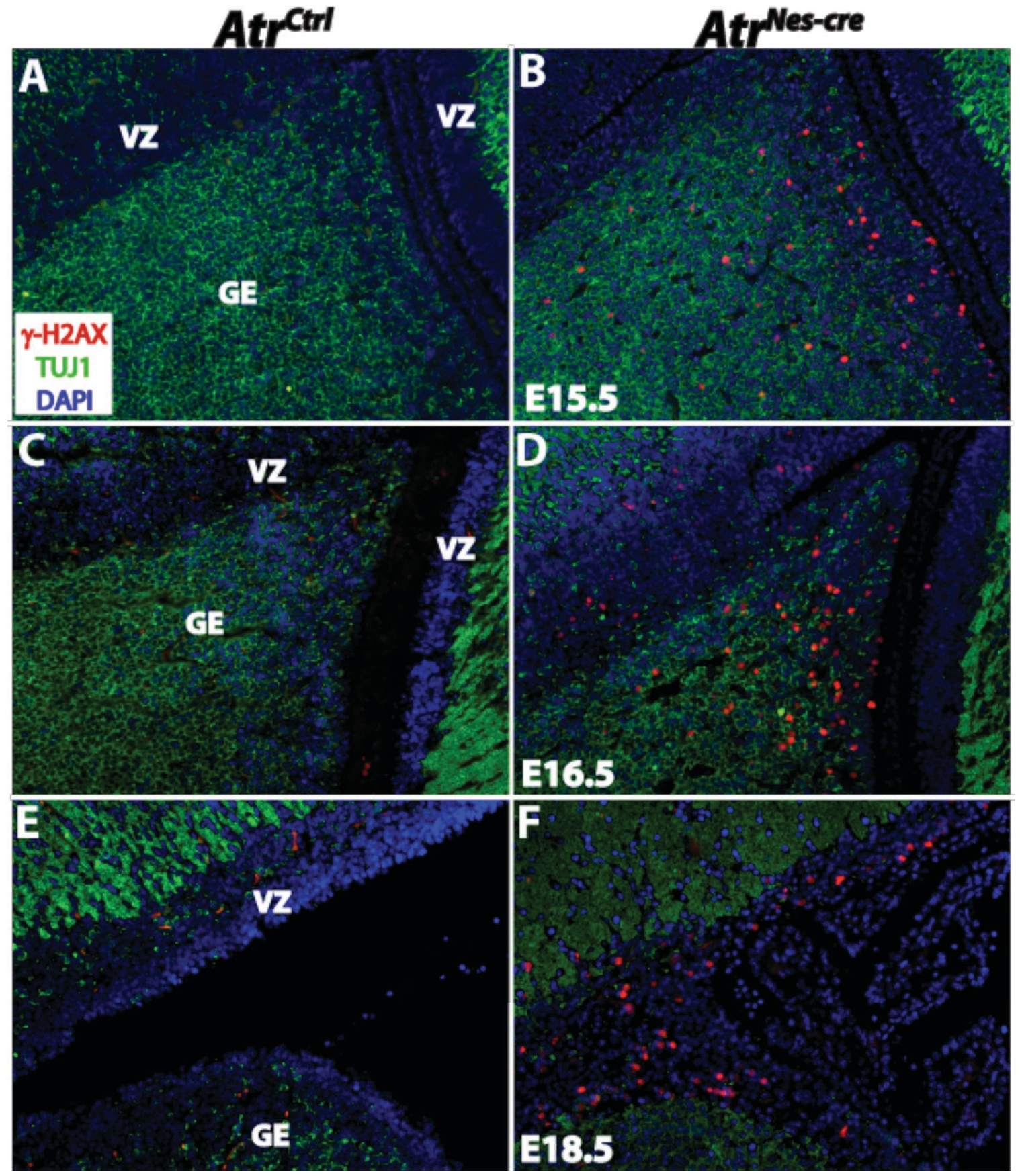

Figure 3.9 Pattern of $\gamma-\mathrm{H} 2 \mathrm{AX}$ in the ganglionic eminence and lateral ventricular zone of embryonic Atr $^{\text {Nes-cre }}$ mice.

$(\mathrm{A}, \mathrm{B})$ At E15.5, the ganglionic eminence (GE) showed substantial $\gamma-\mathrm{H} 2 \mathrm{AX}$ (red) signal. Atr $^{\text {Nes-cre }}$ animals did not show DNA damage in the cortical ventricular zone (VZ) until E16.5 (C,D) and by E18.5 $\gamma$-H2AX was shown throughout the entire cortical VZ and the outer $\beta$ tubulin III (TUJ1) (green) negative region of the GE (E,F). Magnification is 200x. 
Production of calretinin-positive interneurons has been attributed to the caudal ganglionic eminence, while the lateral ganglionic eminence generates specific subclasses of interneurons in the striatum, olfactory bulb, piriform cortex, and amygdala (Corbin et al., 2008). For the sake of simplicity, the GE will be considered as a whole with no emphasis placed upon its divisions in this study. However, subsequent work will be required to determine if loss of Atr in the GE specifically affects the subtypes of interneurons in the forebrain.

The proliferative dorsal wall of the lateral ventricle gives rise to a vast variety of neural precursors that will produce the excitatory projection neurons of the cerebral cortex (Corbin et al., 2008). We predicted that the Atr $^{\text {Nes-cre }}$ animals would begin to show severe damage in the VZ relatively early during embryogenesis, similar to that seen in the EGL at E13.5. However, it was not until E16.5 that significant damage was detected in the dorsal VZ (Figure 3.9 and 3.10). In line with the presence of DNA damage and defective neurogenesis, apoptosis was found to be a prominent occurrence during neurogenesis in Atr $^{\text {Nes-cre }}$ samples. The pattern of cell death was somewhat similar to that of $\gamma$-H2AX foci. Despite the presence of $\gamma$-H2AX and 53BP1 foci in the E13.5 Atr ${ }^{\text {Nes-cre }}$ EGL, apoptosis was not detected using either TUNEL assay or immunostaining against ssDNA in the E13.5 cerebellum (data not shown). The Atr $^{\text {Nes-cre }}$ GE did contain many apoptotic cells, as predicted by the presence of substantial DNA damage in that area at E13.5 (data not shown).

The presence of $\gamma$-H2AX is not necessarily an indicator of apoptosis, as demonstrated by the occurrence of DNA damage foci in the adult Lig $4^{\text {Nes-cre }}$ which did not exhibit apoptosis based upon TUNEL assay (data not shown). However, DNA damage does lead to apoptosis such that the two phenomena are often correlated. Immunostaining against ssDNA (data not shown) and TUNEL assay demonstrated this in $\mathrm{Atr}^{\mathrm{Ctrl}}$ and $\mathrm{Atr}^{\text {Nes-cre }}$ in which apoptosis did largely mirror the pattern of $\gamma-\mathrm{H} 2 \mathrm{AX}$ observed at E15.5 and onward. In the E15.5 $\mathrm{Atr}^{\text {Nes-cre }}$ cerebellum, most cell death was observed in the EGL with some TUNEL-positive cells in the RL (Figure 3.11 upper panels). The level of cell death increased in the E16.5 cerebellum compared to that of the E15.5 $\mathrm{Atr}^{\mathrm{Nes}-}$ ${ }^{c r e}$ samples (Figure 3.11 middle panels). By E18.5, cell death was seen primarily in the IGL of the Atr Nes-cre cerebellum (Figure 3.11 lower panels). This pattern of cell death was relatively consistent with the pattern of DNA damage foci described above. At E18.5, the Atr $^{\text {Nes-cre }}$ cerebellum showed $\gamma$-H2AX signal only in the IGL, similar to the pattern of cell death (data not shown). The same proved true of the GE and VZ in that the occurrence of apoptosis largely correlated with the presence of DNA damage (Figure 3.12 and 3.13).

After DNA damage, both ATM and ATR phosphorylate p53 on serine 15 (serine 18 in mice) (Canman et al., 1998; Cimprich \& Cortez, 2008; Tibbetts et al., 1999). As a critical part of the DNA damage response, p53 can activate either cell cycle arrest or apoptosis (Vousden \& Lu, 2002). In order to better understand the nature of apoptotic induction in Atr $^{\text {Nes-cre }}$ mice, immunostaining against p53-phosphoserine 15 was used. Phosphorylation of p53-serine 15 was detected in the cerebellum, GE and cortical ventricular zone (Figure 3.14) and mimicked the patterns of apoptosis and DNA damage 


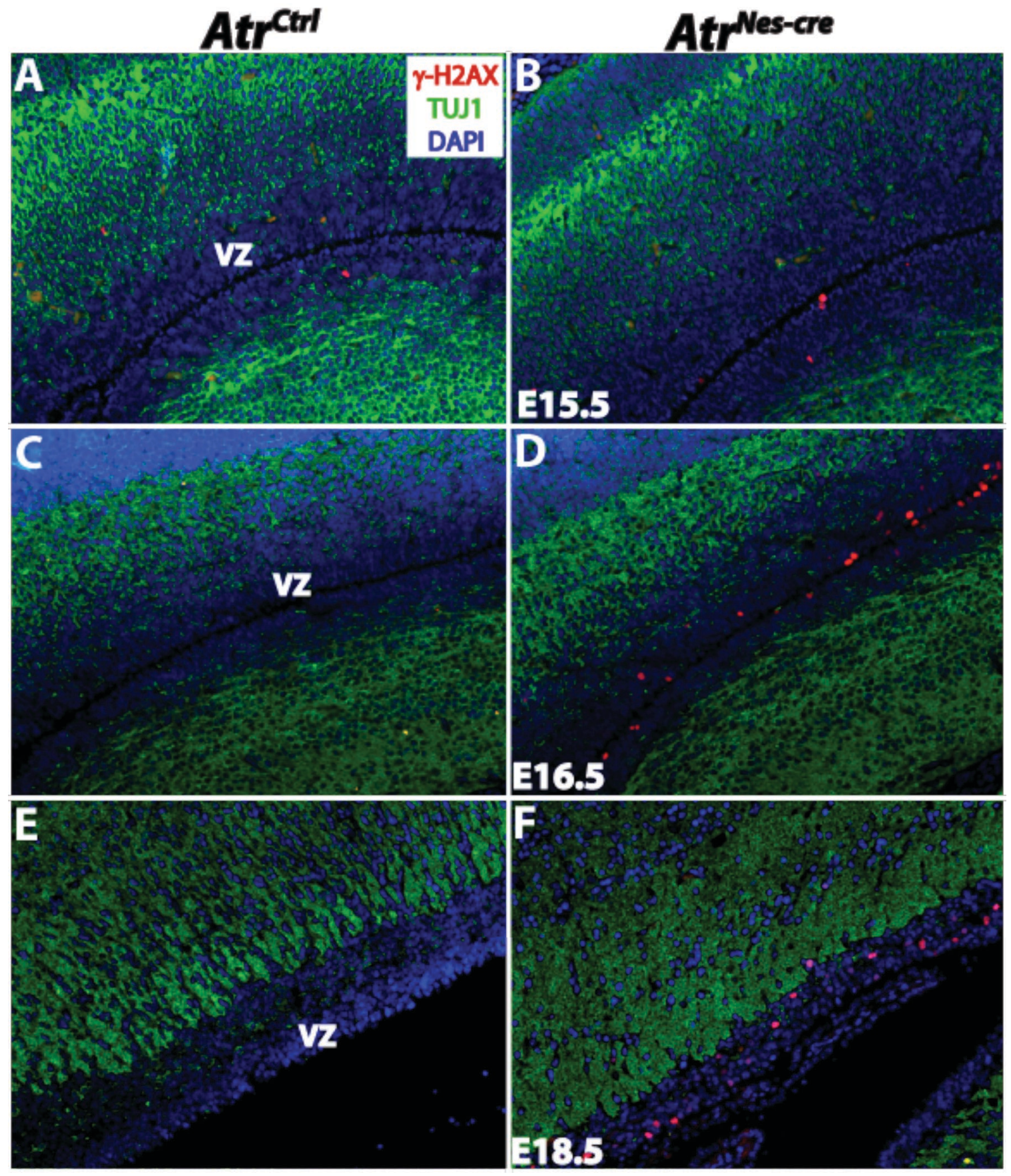

Figure 3.10 Pattern of $\boldsymbol{\gamma}-\mathrm{H} 2 \mathrm{AX}$ formation in the developing Atr $^{\text {Nes-cre }}$ cortex. (A, B)The ventricular zone of the $A t r^{\text {Nes-cre }}$ neopallial cortex showed only a slight increase in $\gamma$-H2AX (red) immunostaining as compared to $A_{t r}{ }^{C t r l}$ at E15.5. (C-F) From E16.5 to E18.5, DNA damage became much more prominent in this region. Labeling against $\beta$ tubulin III (TUJ1) (green) delineated the border between the VZ and the more differentiated sub-ventricular zone. Magnification is 200x. 


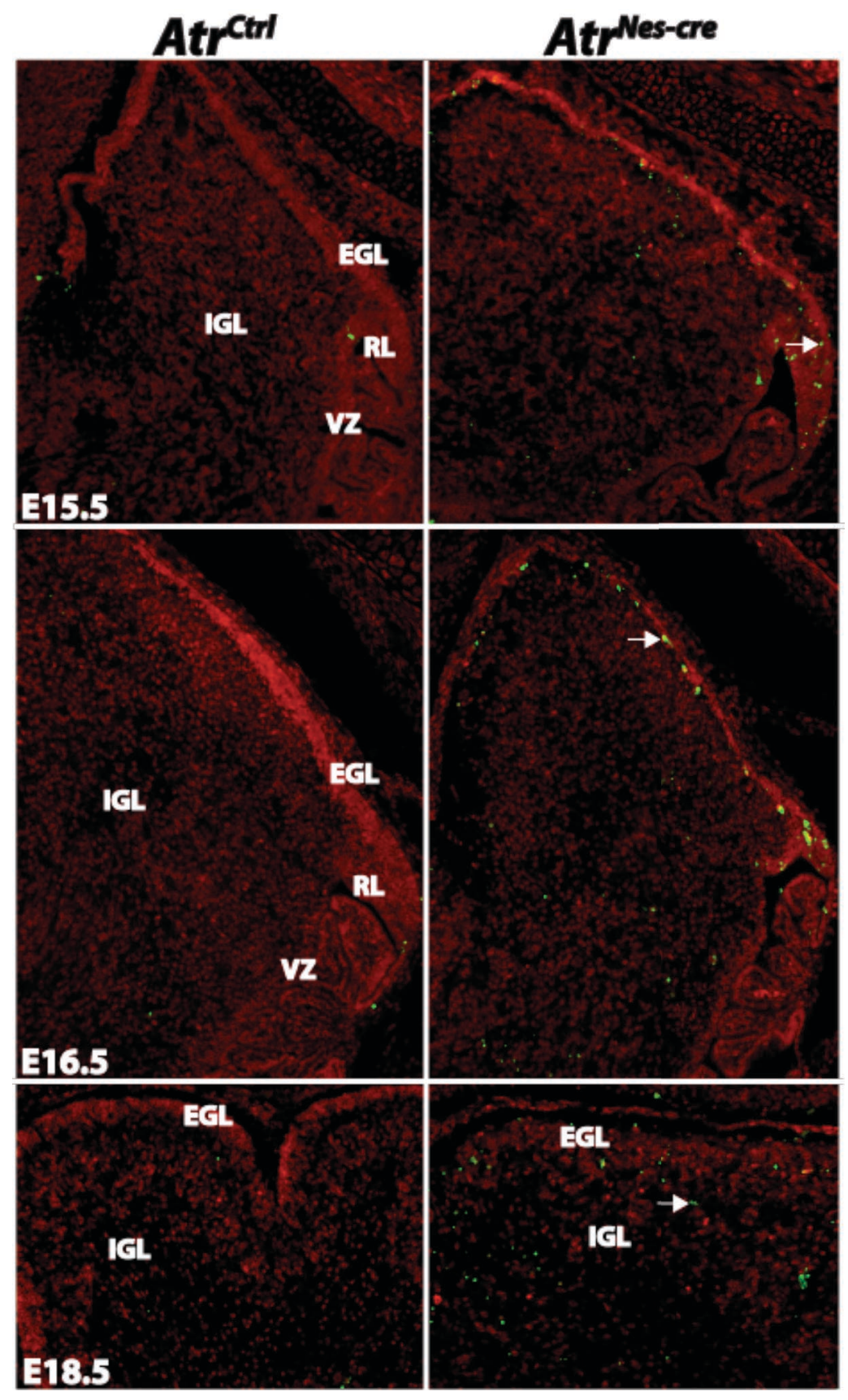

Figure 3.11 Pattern of cell death in the Atr $^{\text {Nes-cre }}$ cerebellum.

TUNEL labeling (green) was used to detect apoptosis in the embryonic Atr ${ }^{\text {Nes-cre }}$ cerebellum. At E15.5, Atr ${ }^{\text {Nes-cre }}$ samples showed increased apoptosis primarily in the external granule layer (EGL). Cell death was also apparent in the rhombic lip (RL) in E15.5 Atr ${ }^{\text {Nes-cre }}$ samples (upper panels). E16.5 Atr ${ }^{\text {Nes-cre }}$ samples showed greater presence of cell death in all regions of the cerebellum, except the dorsal wall of the fourth ventricle (VZ) (middle panels). At E18.5, the RL is diminished and the EGL is fully formed.

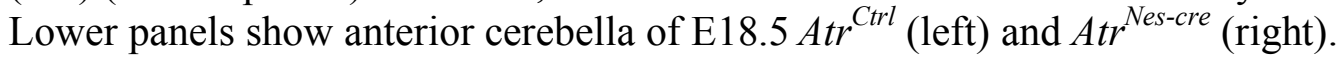
Apoptosis is mainly visible in the IGL. Magnification is 200x. Nuclei were stained with propidium iodide (red). Arrows indicate TUNEL-positive cells. 


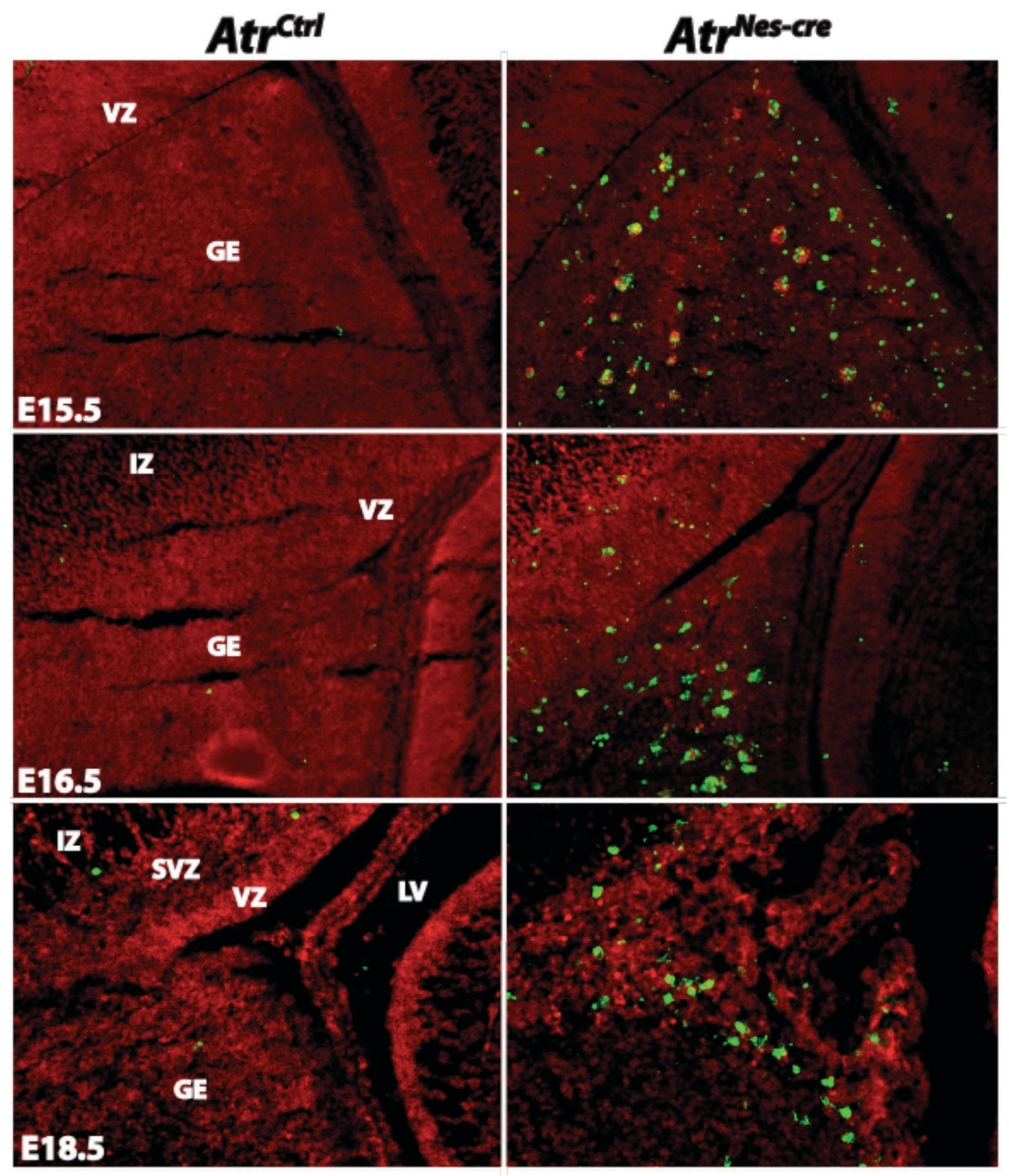

Figure 3.12 Pattern of cell death in lateral ventricular zone and ganglionic eminence of Atr $^{\text {Nes-cre embryos. }}$

At E15.5, the ganglionic eminence (GE) showed substantial TUNEL signal (green) (upper panels). Atr ${ }^{\text {Nes-cre }}$ animals did not show substantial cell death in the cortical ventricular zone (VZ) until E16.5 (middle panels) and by E18.5 TUNEL positive cells were visible throughout the entire cortical VZ and the outer region of the GE (lower panels). Magnification is 200x. Nuclei were stained with propidium iodide (red). Lateral ventricle is abbreviated $\mathrm{LV}$. 


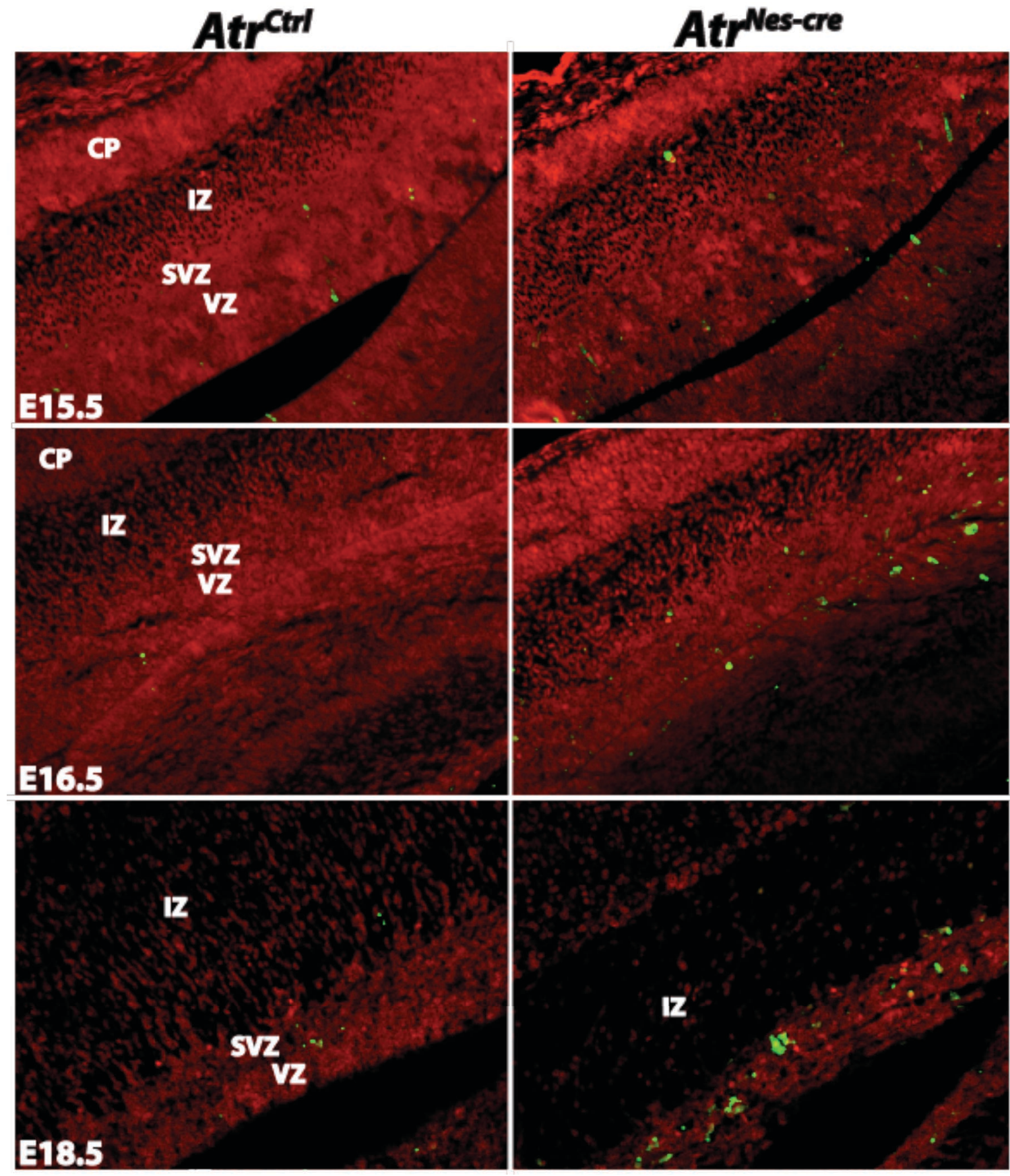

Figure 3.13 Pattern of cell death in the ventricular zone of the embryonic Atr $^{\text {Nes-cre }}$ cortex.

The ventricular zone of the Atr $^{\text {Nes-cre }}$ neopallial cortex showed only a slight increase in TUNEL signal (green) as compared to $A t r{ }^{C t r l}$ at E15.5 (upper panels). From E16.5 to E18.5, DNA damage became much more prominent in this region (middle and lower panels). Magnification is 200x. Nuclei were stained with propidium iodide (red). Abbreviations: ventricular zone (VZ), sub-ventricular zone (SVZ), intermediate zone (IZ), cortical plate (CP). 


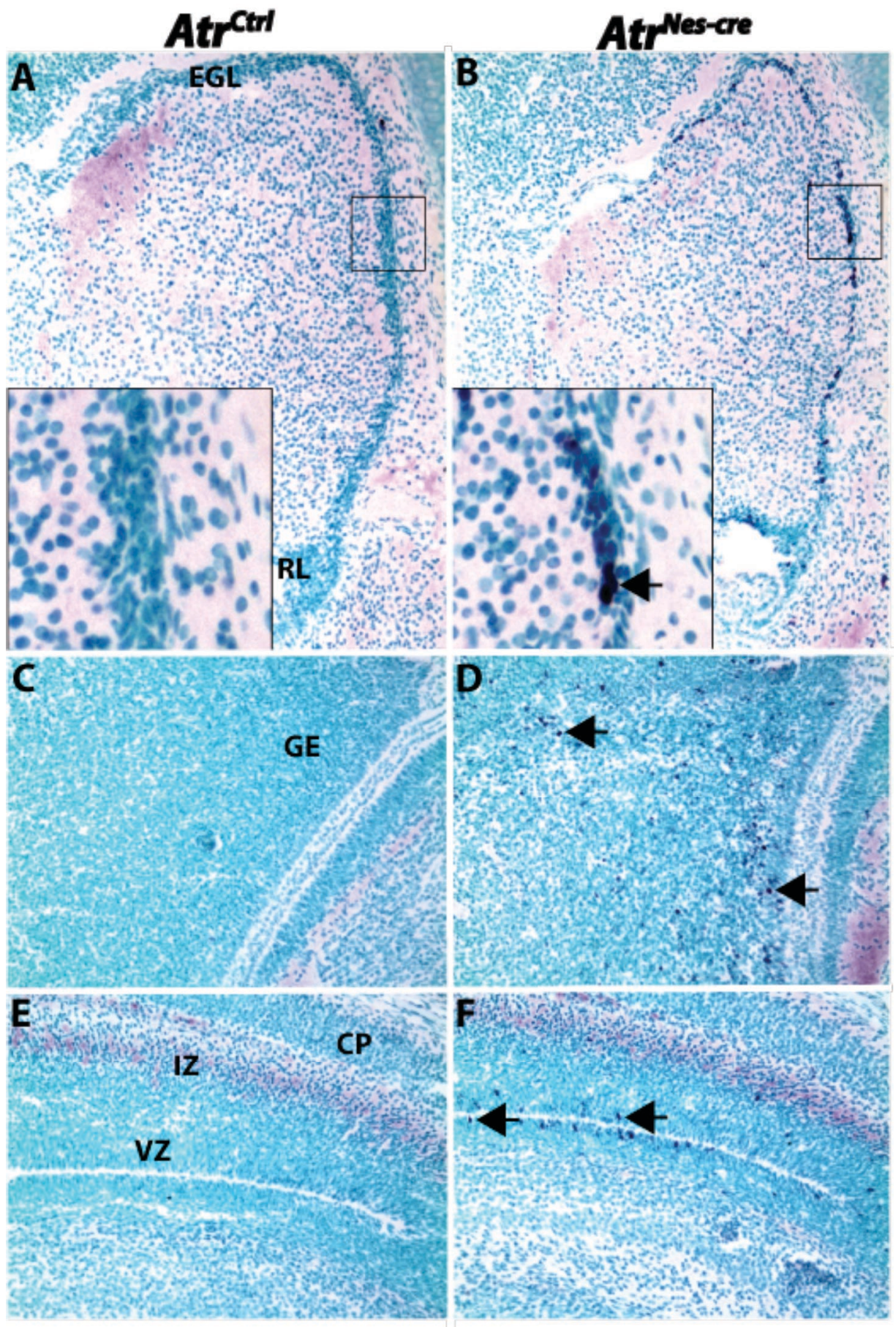

Figure 3.14 Immunostaining against p53-phosphoserine 18 in E16.5 Atr $^{C t r l}$ and Atr $^{\text {Nes-cre }}$ samples.

Activation of $\mathrm{p} 53$ correlated with the incidence of $\gamma-\mathrm{H} 2 \mathrm{AX}$ appearance and apoptosis. (A, B) At E16.5, the Atr ${ }^{\text {Nes-cre }}$ external granule layer (EGL) and rhombic lip (RL) showed upregulation of p53 that was not observed in Atr ${ }^{C t r l}$ animals. (C-F) The same was true in the ganglionic eminence (GE) and the ventricular zone (VZ). The intermediate zone (IZ) and the cortical plate $(\mathrm{CP})$ were negative for p53 induction. Arrows indicate p53 ser 18positive cells. 
observed during development of Atr $^{\text {Nes-cre }}$ embryos (Figures 3.8-3.13). Atr ${ }^{\text {Nes-cre }}$ mice were crossed onto a p53-null background to determine if p53 induction caused microcephaly associated with ATR-Seckel syndrome (Personal communication with Dr. Pierre-Olivier Frappart, March 6, 2008). Dr. Frappart observed full rescue of apoptosis in all affected regions of the Atr $^{\text {Nes-cre }}$ E15.5 animals in the $p 53^{-/-}$genetic background, based upon TUNEL labeling and immunostaining against active caspase-3 (Figure 3.15Ca-f, m-r). However, P5 Atr ${ }^{\text {Nes-cre }} p 53^{-/-}$animals did not exhibit substantial rescue of neuropathology associated with Atr loss (Figure 3.15A-B). Furthermore, Dr. Frappart's data showed no defect in proliferation of granule cell progenitors in the E15.5 Atr $^{\text {Nes-cre }}$ or Atr ${ }^{\text {Nes-cre }} p 53^{-/-}$EGL as indicated by BrdU labeling of S-phase cells (Figure 3.15Cs-u). The same was true for the GE (Figure 3.15Cv-x). These data indicate that though p53dependent apoptosis is a feature of the Atr $^{\text {Nes-cre }}$ phenotype, it does not significantly contribute to the gross pathological state of the brain. Furthermore, p53-induced cell cycle arrest is not a significant contributing factor to the microcephaly and aberrant neurogenesis that is characteristic of the Atr ${ }^{\text {Nes-cre }}$ genotype and hence ATR-SS. These results are currently being independently confirmed.

\subsubsection{Atr deficiency results in depletion of proliferating cell populations during neurogenesis}

Having established the neurogenic defects that result from Atr deficiency in the Atr ${ }^{\text {Nes-cre }}$ model were not due to p53 signaling, embryonic studies were necessary to determine the events responsible for the phenotype. It was clear based upon Dr. Frappart's report of the Atr ${ }^{\text {Nes-cre }} p 53^{-/-}$mice that apoptosis as detected by TUNEL and active-caspase 3 was not the cause of defective neurogenesis, as p53-deficiency rescued apoptosis without correcting neural malformations. Up to E15.5, no proliferation defect was observed in any area of the Atr $^{\text {Nes-cre }} \mathrm{CNS}$ when compared to controls (Figure 3.15C and data not shown). At E16.5 however, a dramatic decrease in PCNA and Ki67 positive cells was apparent in the EGL. The rhombic lip did not appear significantly different in Atr ${ }^{\text {Nes-cre }}$ samples as compared to controls (Figure 3.16). It was surprising that the RL was not more dramatically affected by Atr inactivation, as it is comprised of proliferating neural precursors. To determine if the Atr $^{\text {Nes-cre }}$ genotype affected the proliferating neural precursors of the RL and the fourth ventricle (V4), immunostaining against SOX2 was performed. SOX 2 is a transcription factor required for proliferation and maintenance of neural stem cells (Nicolis, 2007; Zappone et al., 2000). The RL remains prominent until about E17.5, at which time the EGL appears highly developed and the RL is largely diminished. Despite the presence of $\gamma-\mathrm{H} 2 \mathrm{AX}$ and apoptotic cells in the E16.5 Atr ${ }^{\text {Nes-cre }}$ $\mathrm{RL}$, there was no decrease in the number of either SOX2-positive or PCNA- positive cells (Figure 3.16-3.17). No notable differences in either SOX2 expression or expression of the proliferation markers PCNA and Ki67 were observed in the forebrain until later stages of embryonic development. Proliferation of the lateral ventricular zone and RMS appeared largely unaffected in E16.5 and E17.5 Atr ${ }^{\text {Nes-cre }}$ samples (data not shown). At E18.5 there was a drastic difference in PCNA status between Atr $^{C t r l}$ and Atr ${ }^{\text {Nes-cre }}$ ganglionic eminence (GE) and the lateral ventricular zone (Figure 3.18A-E). A more subtle decrease in the number of proliferating cells in the OB was observed (Figure 
Figure 3.15 Apoptosis was rescued in $\mathrm{Atr}^{\mathrm{Nes-cre}} \mathrm{p53^{-/- }}$ animals, but neuropathology was not affected by $\mathrm{p53}$ loss in P5 Atr $^{\text {Nes-cre }}$ mice.

(A) Atr ${ }^{\text {Nes-cre }} p 53^{+/-}$mice showed significantly increased weight in comparison to agematched $A t r^{\text {Nes-cre }}$ littermates $(\mathrm{P}$ value $=0.0063)$. The Atr $^{\text {Nes-cre }} p 53^{-/-}$genotype also positively affected $A t r^{\text {Nes-cre }}$ body weight $(\mathrm{P}$ value $=0.0099)$. Neither the Atr ${ }^{\text {Nes-cre }} p 53^{+/-}$ nor $A t r^{\text {Nes-cre }} p 53^{-/-}$genotype showed a significant difference in brain size compared with age-matched $A t r^{\text {Nes-cre }}$ littermates (P values are 0.0730 and 0.1631 , respectively). (B) The Atr ${ }^{\text {Nes-cre }} p 53^{-/-}$phenotype was indistinguishable from that of $A t r^{\text {Nes-cre }}$ in terms of size (ac) or fine structure of the brain based on H\&E stain (d-i) or immunostaining against calbindin D18K to label cerebellar Purkinje cells (j-o). (C) Sections were collected from E15.5 embryos and evaluated for differences in DNA damage signaling between the Atr ${ }^{\text {Nes-cre }}$ and $\mathrm{Atr}^{\text {Nes-cre }} \mathrm{p} 53^{-/-}$samples. The $p 53^{-/-}$background rescued apoptosis based on TUNEL labeling (a-f) and immunostaining against active-caspase 3 (m-r).

Immunostaining against $\gamma$-H2AX showed persistent DNA damage in $\mathrm{Atr}^{\text {Nes-cre }} \mathrm{p} 53^{-/-}$

embryos (g-1). BrdU labeling revealed no impact of either the Atr $^{\text {Nes-cre }}$ or Atr ${ }^{\text {Nes-cre }} p 53^{-/-}$ upon proliferation at E15.5 (s-x). Immunostaining against p53 confirmed its stabilization in $A t r^{\text {Nes-cre }}$ samples and showed its inactivation in $A t r^{\text {Nes-cre }} p 53^{-/-}$samples $(\mathrm{y}-\varepsilon)$. Personal communication with Dr. Pierre-Olivier Frappart, March 6, 2008. 
A

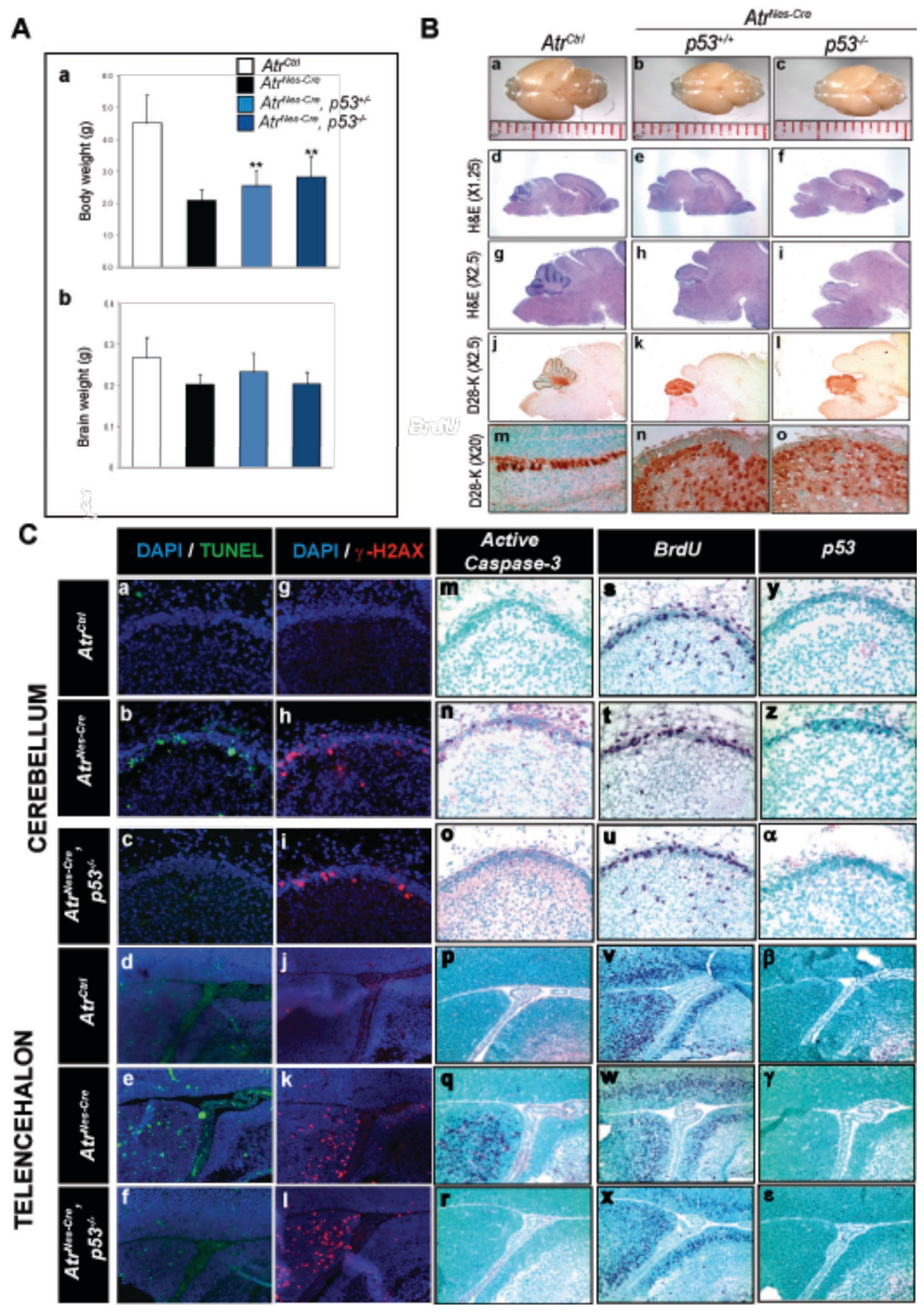

B 

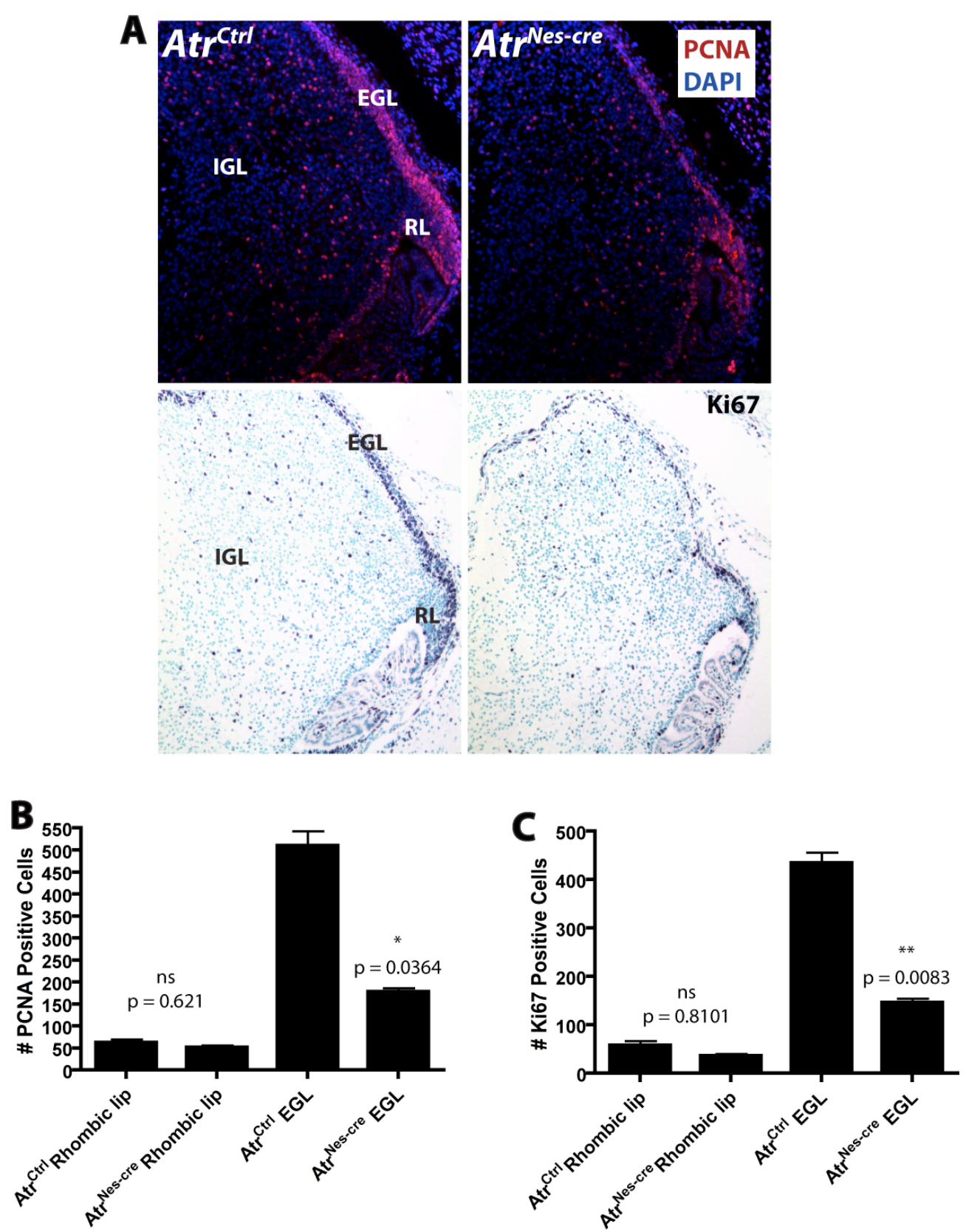

Figure 3.16 Proliferation of the EGL in the E16.5 $\mathrm{Atr}^{\text {Nes-cre }}$ cerebellum is decreased. (A) Immunostaining against PCNA (upper panels) and Ki67 (lower panels) was used to

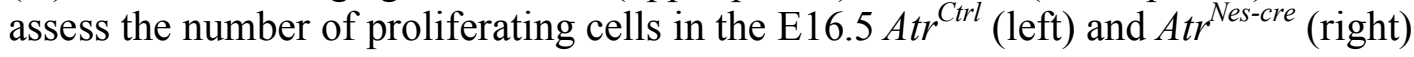
cerebella. (B) The total number of PCNA-positive cells was counted from images representative of those shown in A. (C) The total number of Ki67-positive cells was counted from images representative of those shown in A. Multiple sections from each sample were analyzed. Three animals per genotype were analyzed. P value was derived from an unpaired t-test. Photomicrographs were captured at 200x magnification. 


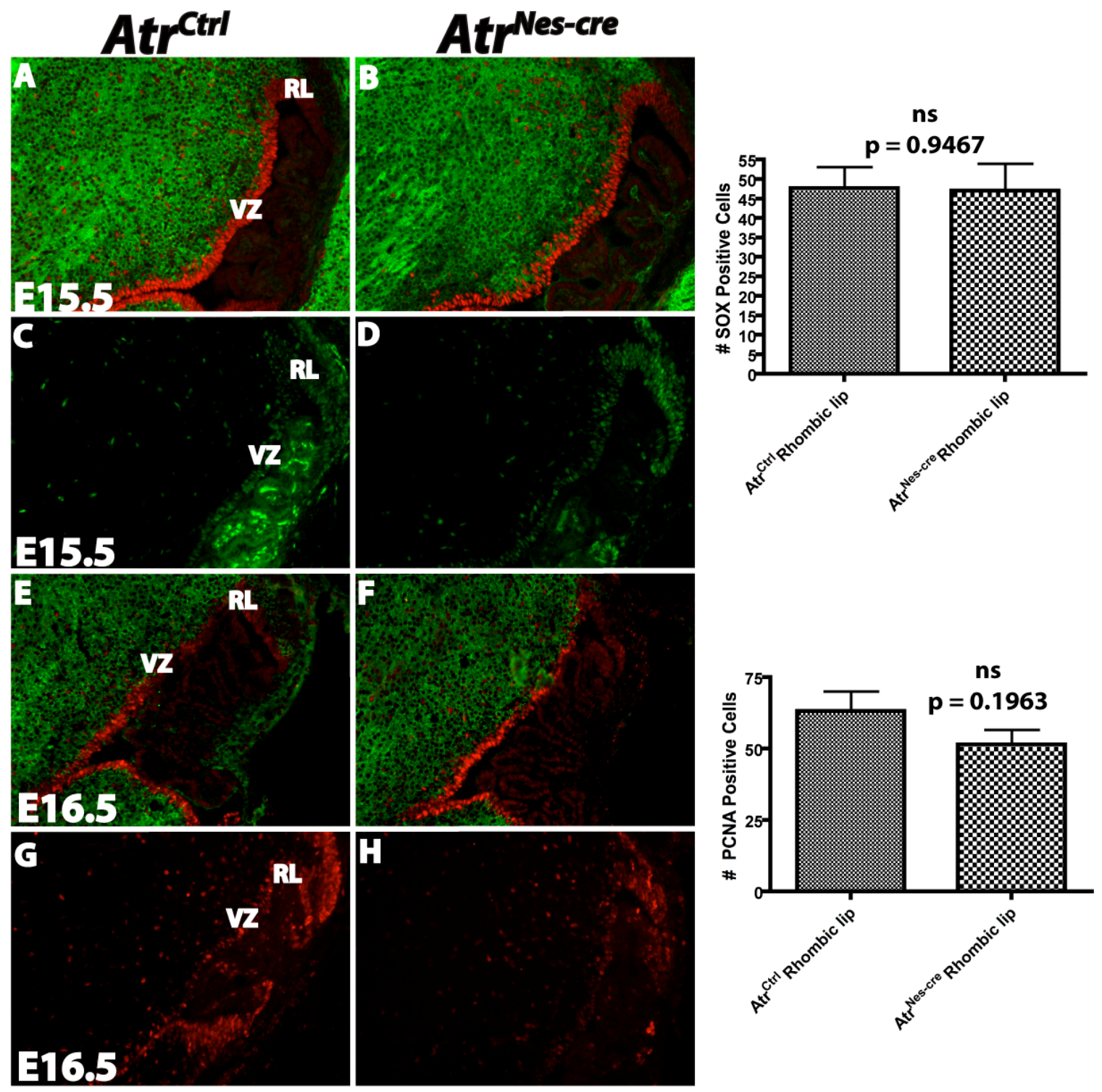

Figure 3.17 The rhombic lip is largely unaffected in Atr $^{\text {Nes-cre }}$ embryos.

Immunostaining against SOX2 (red) and $\beta$ tubulin III (TUJ1) (green) was used to analyzed the effects of the Atr ${ }^{\text {Nes-cre }}$ genotype on cerebellar precursor cells in E15.5 (A,B) and E16.5 (E, F) samples. Immunostaining of E15.5 Atr ${ }^{\text {Ctrl }}$ and Atr $^{\text {Nes-cre }}$ samples against PCNA (green) indicated no difference in proliferation in the RL at this stage $(C, D)$. Similarly, there was no change in the number of PCNA-positive cells (red) in the RL of $A t{ }^{C t r l}$ and $A t r^{\text {Nes-cre }}$ samples at E16.5 $(\mathrm{G}, \mathrm{H})$. Quantitation of these results (as described in Figure 3.14) was performed for E16.5 samples (right hand panels). Photomicrographs were captured at 200x magnification. 

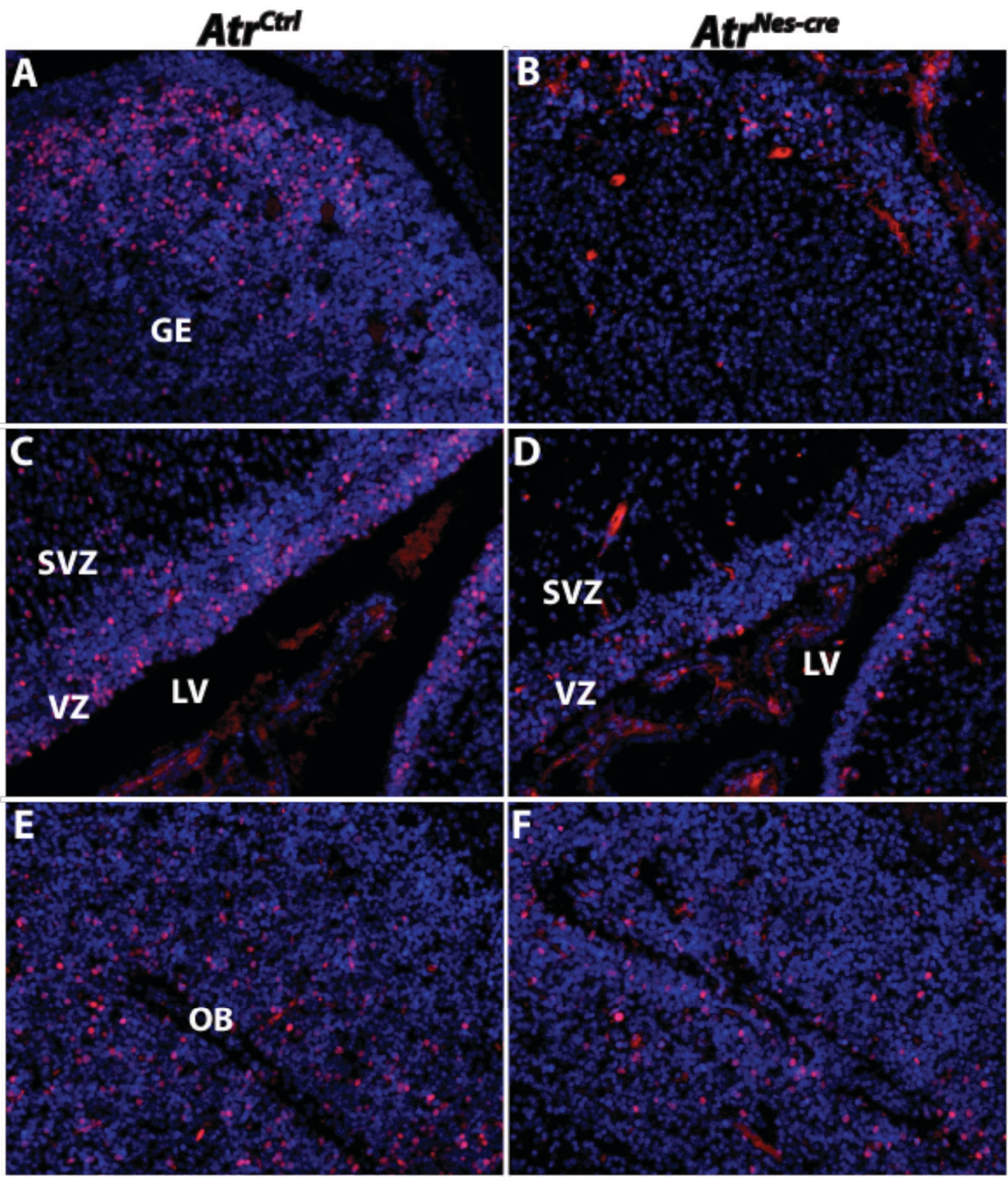

Figure 3.18 Immunostaining against PCNA in E18.5 Atr $^{\text {Nes-cre }}$ forebrain.

Immunostaining against PCNA (red) showed decreased proliferation in the Atr $^{\text {Nes-cre }}$ forebrain at E18.5. Proliferating regions such as the ganglionic eminence (GE), the walls of the lateral ventricle (VZ), the subventricular zone (SVZ) and the rostral migratory stream of the olfactory bulb (OB) were affected. Nuclei were stained with DAPI (blue). Magnification is 200x. The lateral ventricle is abbreviated LV. 
3.18E-F). Immunostaining against Ki67 was consistent with those findings (data not shown). Immunostaining against PCNA revealed substantial depletion of proliferating cells in the lateral ventricular zone of $\mathrm{P} 5 \mathrm{Atr}{ }^{\text {Nes-cre }}$ animals, in comparison to littermate controls (Figure 3.19A-B). The Atr ${ }^{\text {Nes-cre }}$ cerebellar EGL also had significantly fewer proliferating cells during post-natal life, consistent with the embryonic findings. This was particularly true in more lateral sections (Figure 3.19C-D) and less dramatic (though obvious compared to $A t r^{C t r l}$ ) in medial regions that displayed a relatively thicker EGL with more proliferating cells (Figure 3.19E-F). The $A t r^{\text {Nes-cre }}$ dentate gyrus showed only a marginal decrease in PCNA positive cells compared to that of Atr $^{C t r l}$ mice (data not shown). We therefore attribute the neurogenesis defects found in tr $^{\text {Nes-cre }}$ to reduced proliferation, rather than apoptosis, in the developing nervous system.

\subsubsection{Atm and Atm functions are not obviously redundant in the developing brain}

The induction of p53-dependant cell death in proliferating regions of the Atr $^{\text {Nes-cre }}$ embryonic brain indicated that either ATR signaling is not required for p53 activity in these regions or that ATM is responsible for p53 activation in proliferating neural precursors in the absence of ATR. Alternatively there may be another gene involved in p53 regulation during the DNA damage response.

To genetically delineate the in vivo distinctions between ATM and ATR activity during neurogenesis, Atm was conditionally deleted in the CNS through generation of $A t m^{\text {loxP } / o x P}$ mice which were then crossed with $A t r^{+/ l o x P}$; Nestin-cre mice to produce Atr ${ }^{\text {loxPlloxP }} ;$ Atm $^{\text {loxPlloxP }}$; Nestin-cre $\left(\right.$ Atr $^{\text {Nes-cre }}$ Atm $\left.^{\text {Nes- }}{ }^{\text {cre }}\right)$ double conditional knock-out animals. The conditional Atm allele was chosen for this study in order to circumvent possible affects outside the nervous system as well as to promote more efficient breeding strategies (germline $\mathrm{Atm}^{-/}$animals are sterile). $\mathrm{Atm}^{\text {Nes-cre }}$ mice had not been previously characterized, thus it was necessary to first confirm the ablation of Atm activity in the nervous system. At the time these animals were generated, there was not a reliable antibody against ATM suitable for use in murine tissue. So, we used real-time PCR to confirm excision of the DNA encoding the targeted exon and assessed the effects of the $\mathrm{Atm}^{\text {Nes-cre }}$ genotype on IR-induced apoptosis in the post-natal cerebellum. DNA extracted from cryosections of E15.5 $\mathrm{Atr}^{\text {Nes-cre }} \mathrm{Atm}^{\text {Nes-cre }}$ brains was used for real-time PCR analysis of excision efficiency for exon 58, which is the floxed exon of the Atm conditional mouse model used in this study. In comparison to one $\mathrm{Atr}^{+/ l o x P} \mathrm{Atm}^{+/ \text {loxP }}$; Nestin-cre $\left(\mathrm{Atr}^{+/ N e s-c r e} \mathrm{Atm}^{+/ \text {Nes-cre }}\right)$ three $\mathrm{Atr}^{\text {Nes-cre }} \mathrm{Atm}^{\text {Nes-cre }}$ showed approximately $70-80 \%$ deletion efficiency (Figure 3.20A). To confirm adequate decrease of Atm activity, P6 Atm ${ }^{\mathrm{Ctrl}}$ and Atm $^{\text {Nes-cre }}$ animals were subjected to 18 Gy IR and allowed to recover for 18 hours prior to sacrifice. TUNEL assay revealed abrogated apoptosis in the EGL and dentate gyrus (data not shown) of the $\mathrm{Atm}^{\text {Nes-cre }}$ animals (Figure 3.20B). Untreated $\mathrm{Atr}^{\mathrm{Ctrl}} \mathrm{Atm}^{\text {Nes-cre }}$ samples were identical to untreated $A t{ }^{C t r l}{ }^{C t m}{ }^{C t r l}$ littermates in every aspect analyzed. $\mathrm{Atr}^{\mathrm{Ctrl}} \mathrm{Atm}^{\text {Nes-cre }}$ controls have been excluded from some figures for more efficient use of space. 

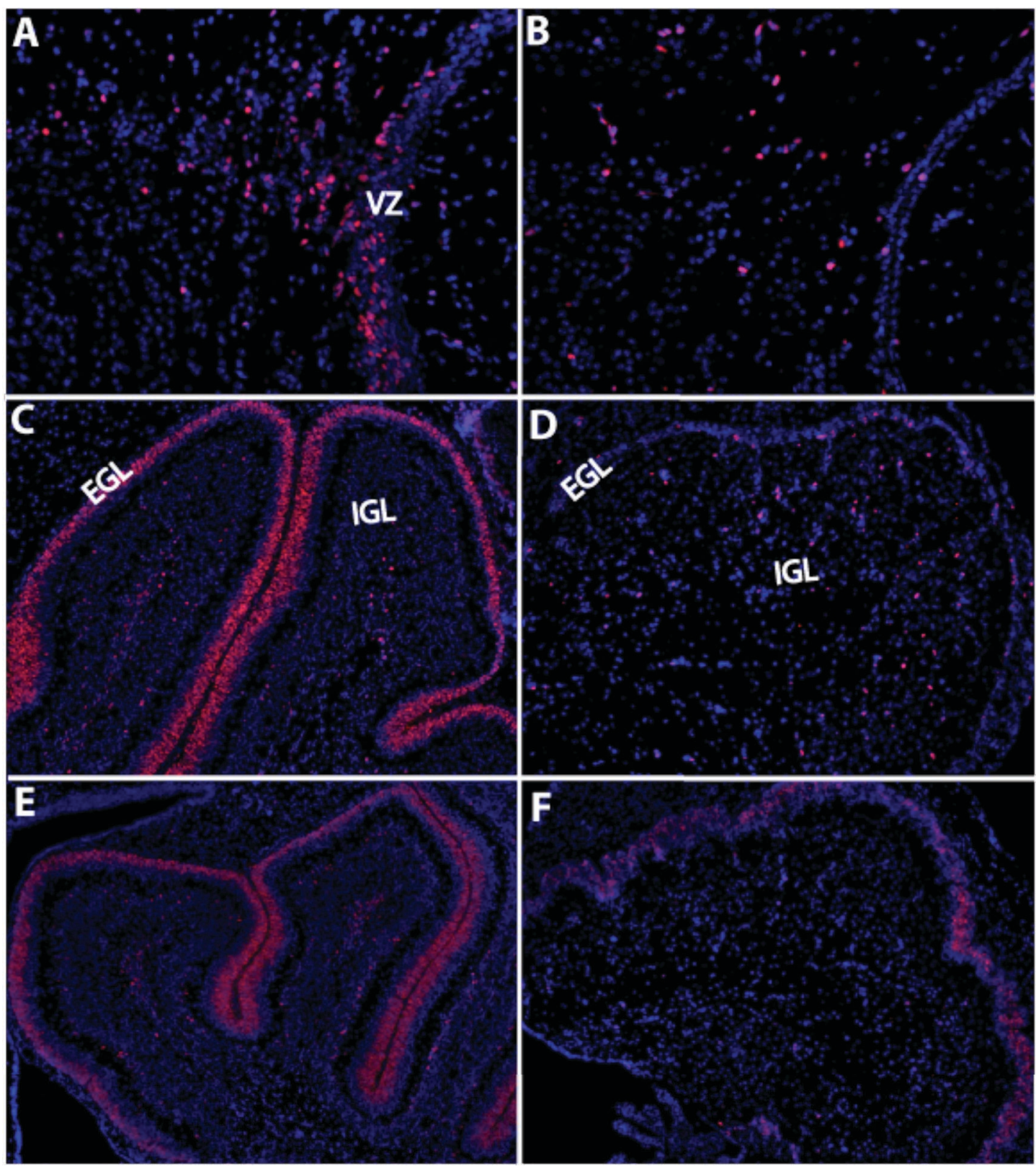

Figure 3.19 Immunostaining against PCNA in P5 Atr $^{C t r l}$ and Atr $^{\text {Nes-cre }}$ sagittal sections of cerebellum and forebrain.

Immunostaining against PCNA (red) was applied to brain sections collected from $\mathrm{Atr}$ Ctrl and Atr $^{\text {Nes-cre }}$ mice at P5. Similar to results obtained at E18.5 (Figure 3.16), the walls of the lateral ventricle showed drastically decreased PCNA positivity in the developing cortex and basal ganglia of Atr $^{\text {Nes-cre }}$ samples (A, B). The EGL of the lateral cerebellum was nearly non-existent and showed almost no PCNA-positive cells in the Atr $^{\text {Nes-cre }}$ samples, in stark contrast to that of age-matched $A t{ }^{C t r l}$ animals (C, D). The EGL of the medial cerebellum was thicker than in the lateral sections, but still showed decreased presence of PCNA-positive cells compared to controls (E, F). Nuclei were stained with DAPI (blue). Magnification is 200x in panels A and B, 100x in C-F. 

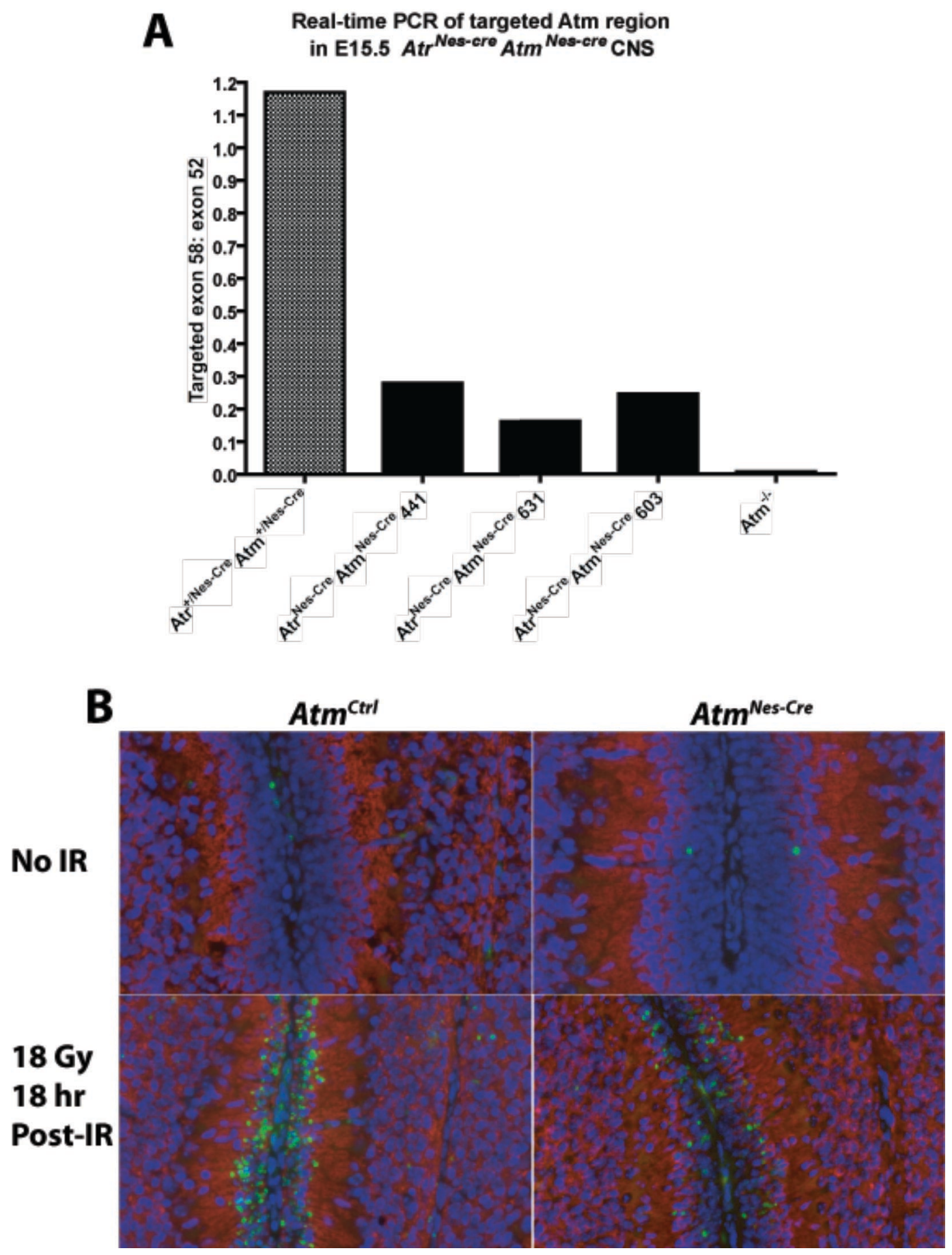

Figure 3.20 Efficient Nestin-cre mediated excision of $\mathrm{Atm}$ exon 58 and confirmation of genotype consistent with $\mathrm{Atm}^{-/-}$mice.

(A) Real-time PCR was used to ascertain deletion efficiency of exon 58 in the $\mathrm{Atm}^{\text {Nes-cre }}$ E15.5 CNS. Results for exon 58 were normalized to those for exon 52. (B) P6 $\mathrm{Atm}^{\mathrm{Ctrl}}$ and $\mathrm{Atm}^{\text {Nes-cre }}$ mice were treated with $18 \mathrm{~Gy}$ of IR or mock irradiated and allowed a recovery period of 18 hours. TUNEL assay (green) and immunostaining against $\beta$ tubulin III (TUJ1) (red) were performed. Nuclei were stained with DAPI (blue). Magnification is 200x. 
$A t r^{\text {Nes-cre }} \mathrm{Atm}^{\text {Nes-cre }}$ mice were born at the expected Mendelian ratio (data not shown), and were phenotypically indistinguishable from $A t r^{\text {Nes-cre }}$ littermates (Figure 3.21). No difference was observed in either the gross appearance or histological features between the $\mathrm{Atr}^{\text {Nes-cre }} \mathrm{Atm}^{\mathrm{Ctrl}}$ and $\mathrm{Atr}^{\text {Nes-cre }} \mathrm{Atm}^{\text {Nes-cre }}$ brains (Figure 3.21A). Neither brain nor body weights showed any significant difference (Figure 3.21B). Lifespan and capacity to feed properly were similarly unaffected in Atr $^{\text {Nes-cre }}$ Atm $^{\text {Nes-cre }}$ pups compared to $A t r^{\text {Nes-cre }}$ Atm ${ }^{\text {Ctrl }}$ littermates (data not shown). Immunostaining against calbindin-D28K showed the cerebellar abnormalities typical of Atr $^{\text {Nes-cre }}$ mice to be unaffected by Atmdeficiency (Figure 3.21C). Other morphologic abnormalities described in section 3.3.1 for $A t r^{\text {Nes-cre }}$ mice were identical to those found in $\mathrm{Atr}^{\text {Nes-cre }} \mathrm{Atm}^{\text {Nes-cre }}$ animals (data not shown). We compared the endogenous DNA damage response between $\mathrm{Atr}^{\mathrm{Nes}-\mathrm{cre}} \mathrm{Atm}^{\mathrm{Crl}}$ and $\mathrm{Atr}^{\text {Nes-cre }} \mathrm{Atm}^{\text {Nes-cre }}$ littermates as well. Immunostaining against total p53 revealed its stabilization in the E16.5 EGL of both $\mathrm{Atr}^{\text {Nes-cre }} \mathrm{Atm}^{\mathrm{Ctrl}}$ and $\mathrm{Atr}^{\text {Nes-cre }} \mathrm{Atm}^{\text {Nes-cre }}$ samples, while no p53-positive cells were observed in $\mathrm{Atr}^{\mathrm{Ctrl}} \mathrm{Atm}^{\mathrm{Ctrl}}$ and $\mathrm{Atr}^{\mathrm{Ctrl}} \mathrm{Atm}^{\mathrm{Nes}-\mathrm{cre}}$ samples (Figure 3.22A-C and data not shown). Phosphorylation of p53 on serine 18 observed in the EGL of $\mathrm{Atr}^{\text {Nes-cre }} \mathrm{Atm}^{\mathrm{Ctrl}}$ embryos was entirely abrogated by Atm-deficiency (Figure 3.22D-F). This leads us to speculate that the p53-phosphoserine 18 positive cells observed in the embryonic EGL of Atr ${ }^{\text {Nes-cre }}$ mice likely reflect granule precursor cells that acquired damage during later stages of replication, but did not initiate the DSB response until after exiting the cell cycle. However, further experiments would be required to prove this to be the case, rather than Atm induction in replicating neural precursors. TUNEL assay and immunostaining against Ki67 were used to determine if Atm loss affected either apoptosis or proliferation in Atres-cre embryos. At E16.5, no significant difference was observed in the levels of either apoptosis or proliferation between Atr $^{\text {Nes-cre }}$ Atm $^{\text {Ctrl }}$ and Atr $^{\text {Nes-cre }}$ Atm $^{\text {Nes-cre }}$ animals, as demonstrated in the EGL

(Figure 3.22G-L). Similarly, no differences in proliferation or apoptosis were observed in the RL, cortex, ganglionic eminence, or other areas in the $\mathrm{Atr}^{\text {Nes-cre }} \mathrm{Atm}^{\text {Nes-cre }}$ nervous system when compared with age-matched $A t r^{\text {Nes-cre }}$ Atm ${ }^{\text {Ctrl }}$ embryos. Phosphorylation of H2AX on ser 139 was also similar between $\mathrm{Atr}^{\text {Nes-cre }} \mathrm{Atm}^{\text {Nes-cre }}$ and $\mathrm{Atr}^{\text {Nes-cre }} \mathrm{Atm}^{\mathrm{Ctrl}}$ embryos (data not shown). These data suggest that ATR and ATM do not function redundantly in the developing nervous system, but may cooperate indirectly to ensure genomic stability.

\subsection{Discussion}

ATM and ATR are structurally and functionally similar signaling kinases that share many overlapping substrates and are involved in the DNA damage response. However, the physiologic requirements of these genes are quite distinct as ATRdeficiency is associated with developmental microcephaly and ATM inactivation leads to the neurodegenerative syndrome A-T (Cimprich \& Cortez, 2008). Activation of ATR occurs through recognition of RPA-coated ssDNA regions and interaction with TopBP1 and ATRIP; whereas current knowledge of ATM activation revolves around its being recruited to DSB sites and undergoing autophosphorylation events that are partially 

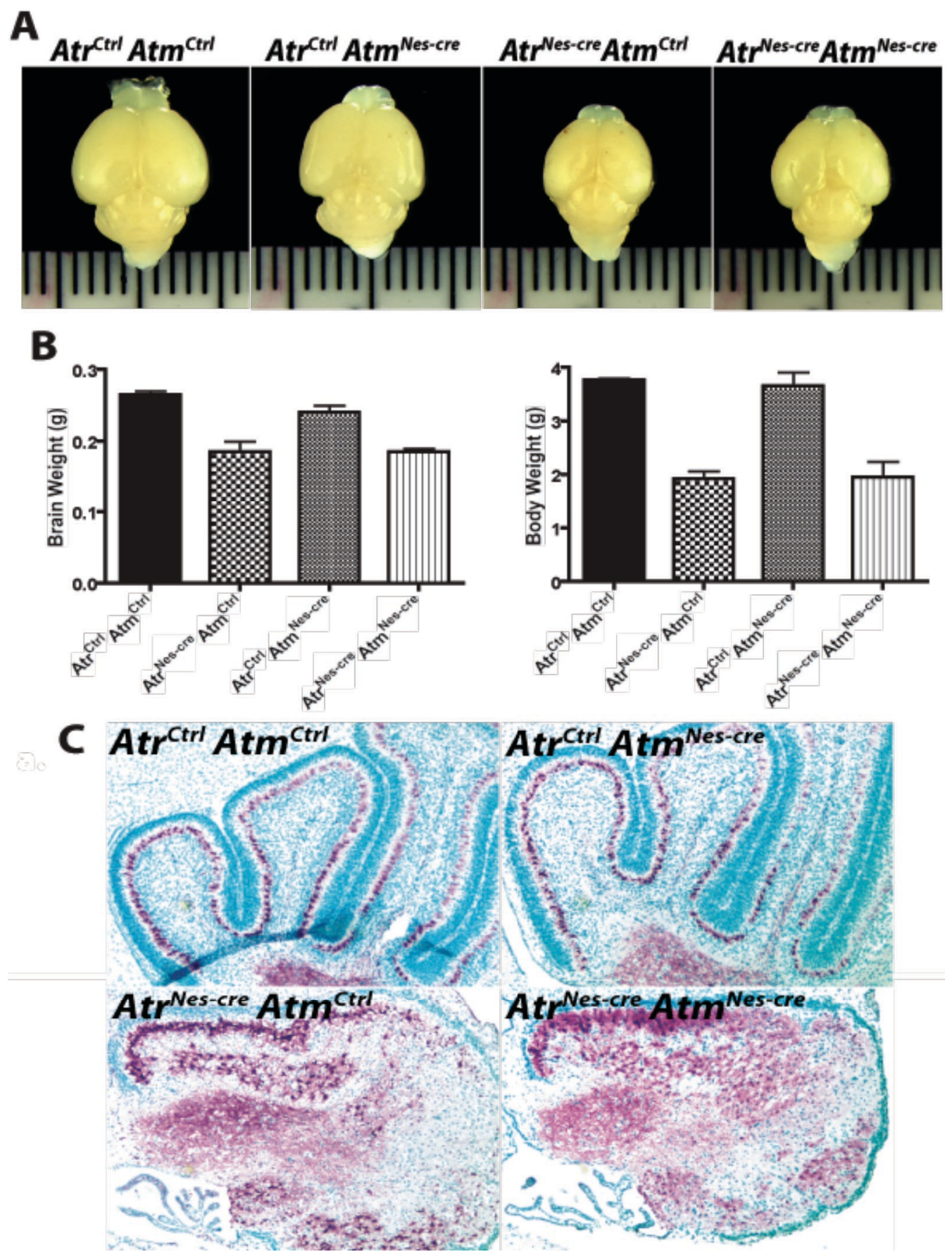

Figure 3.21 $\mathrm{Atr}^{\text {Nes-cre }} \mathrm{Atm}^{\text {Nes-cre }}$ mice are indistinguishable from $\mathrm{Atr}^{\text {Nes-cre }}$ littermates. (A) Measurement of the Atm ${ }^{\text {Nes-cre }}$ mutation towards Atr ${ }^{\text {Nes-Cre }}$ brain size. (B) Brain and body weights were collected from sex-matched cohorts at P5. Error bars represent standard deviation. P value $=1$ between the Atr $^{\text {Nes-Cre }}$ Atm $^{C t r l}$ brain weights and the Atr ${ }^{\text {Nes- }}$ ${ }^{C r e} A^{A m}{ }^{\text {Nes-cre }}$ brain weights. P value $=0.09228(\mathrm{n}=3)$ between the Atr $^{\text {Nes-Cre }}$ Atm $^{\text {Ctrl }}$ body weights and the Atr ${ }^{\text {Nes-Cre }}$ Atm $^{\text {Nes-cre }}$ body weights. (C) Atr ${ }^{\text {Nes-cre }}$ Atm $^{\text {Nes-cre }}$ cerebellar morphology was largely similar to that of $\mathrm{Atr}^{\text {Nes-cre }} \mathrm{Atm}^{\mathrm{Ctrl}}$ littermates, as shown by immunostaining against calbindin-D28K. Methyl green was used to counterstain nuclei. Magnification is $100 x$. 

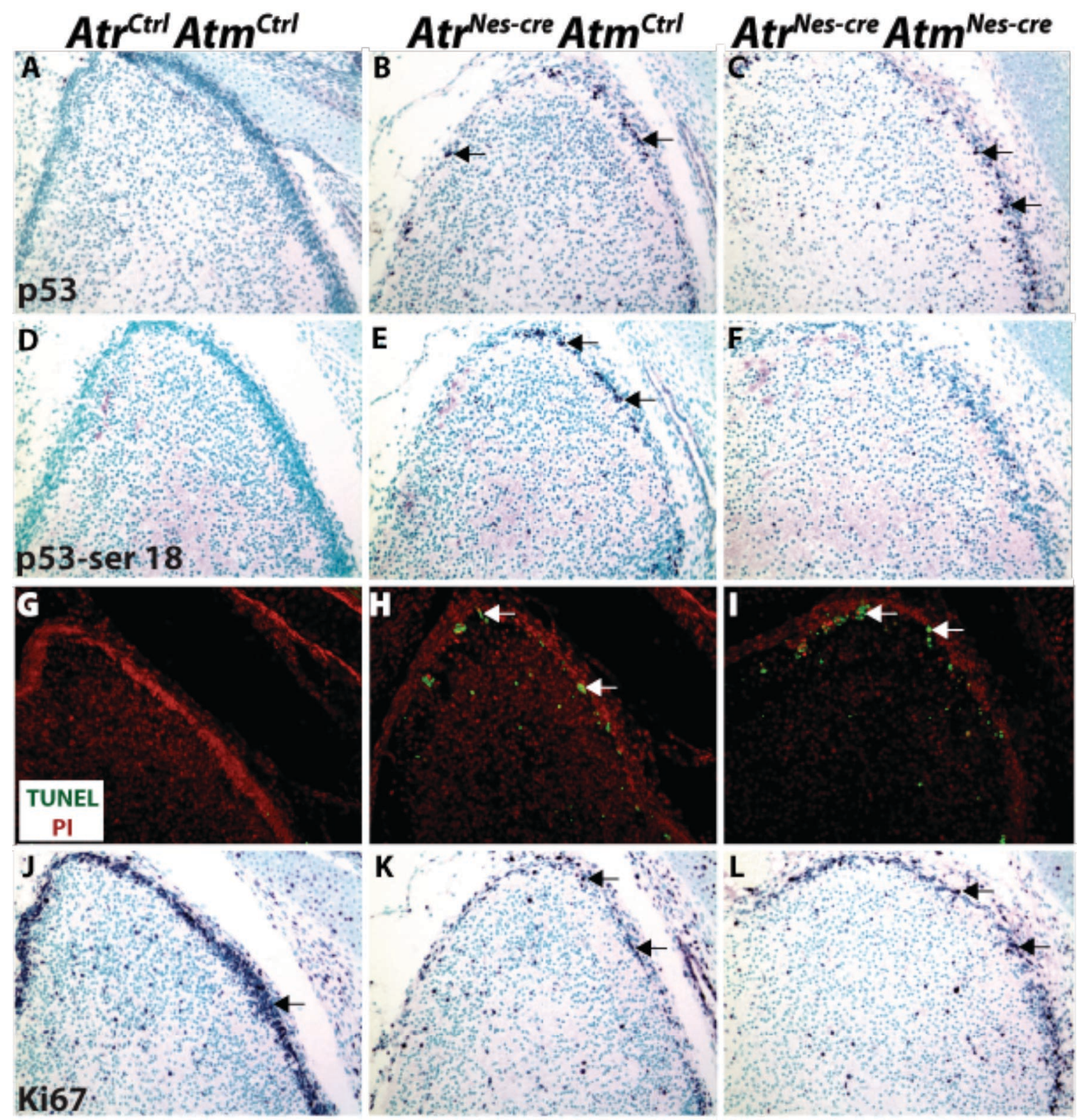

Figure 3.22 DNA damage signaling in the E16.5 Atr $^{\text {Nes-cre }} \mathrm{Atm}^{\text {Nes-cre }}$ EGL.

(A-C) Immunostaining against p53 (purple) showed similar stabilization of total p53 in E16.5 Atr $^{\text {Nes-cre }}$ Atm $^{\text {Ctrl }}$ compared to Atr $^{\text {Nes-cre }}$ Atm $^{\text {Nes-cre }}$ littermates. (D-E) Phosphorylation of p53 serine 18 (purple) was largely abrogated in Atr $^{\text {Nes-cre }}$ Atm $^{\text {Nes-cre }}$ samples. (G-I) TUNEL assay showed that Atm-deficiency did not significantly block apoptosis in the $\mathrm{Atr}^{\text {Nes-cre }} \mathrm{Atm}^{\text {Nes-cre }}$ EGL, as cell death was similar to that observed in $\mathrm{Atr}^{\text {Nes-cre }} \mathrm{Atm}^{\text {Ctrl }}$ samples. (J-I) Immunostaining against Ki67 revealed no effect of Atm-deficiency on proliferation in the E16.5 EGL. Because cerebellar sections are not matched in this figure, no conclusions can be drawn regarding structural differences between any of the genotypes. No differences were observed between $\mathrm{Atr}^{\mathrm{Ctrl}} \mathrm{Atm}^{\mathrm{Ctrl}}$ and $\mathrm{Atr}^{\mathrm{Ctrl}} \mathrm{Atm}^{\text {Nes-cre }}$ samples (data not shown). Magnification is 200x. 
dependant upon the MRN complex (Mordes \& Cortez, 2008; Mordes et al., 2008). For example, ATM-phosphoserine 1981 foci are largely reduced in Nbs1 mutant cells (Kitagawa et al., 2004). Phosphorylation of p53 at serine 15 is an integral component of the DNA damage response. It has been shown that ATM is an important mediator of p53 induction both in vitro and in vivo (Banin et al., 1998; Canman et al., 1998; Kastan et al., 1992; Khanna et al., 1998; Lavin, 2008). ATR function has also been linked to p53 activity. Doxycycline induced overexpression of catalytically inactive ATR in human fibroblasts inhibited p53 phosphorylation on serines 15 and 37 (Tibbetts et al., 1999). Lenti-cre- infected $A T R^{\text {flox/- }}$ fibroblasts showed reduced p53-serine 18 phosphorylation at later time points following IR, with ATM being responsible at earlier time points (Brown \& Baltimore, 2003).

The Atr ${ }^{\text {Nes-cre }}$ phenotype provided insight into the nature of developmental delay and microcephaly associated with ATR-Seckel syndrome. Consistent with the known requirements of ATR in responding to replication stress and UV-induced DNA damage, DSBs associated with p53-dependant apoptosis were observed in areas of the developing Atr ${ }^{\text {Nes-cre }}$ brain that are largely populated by proliferating progenitor cells. Importantly, Atr-deficiency also led to a significant decrease in the number of proliferating cells in the forebrain and cerebellar EGL of Atr ${ }^{\text {Nes-cre }}$ embryos. Surprisingly, this affect was not observed in the rhombic lip, which is comprised of proliferating progenitor cells that generate the EGL. Additionally, it was surprising that the forebrain did not show the decrease in proliferation until E18.5. As the appearance of DSBs, apoptosis, and decreased number of proliferating progenitor cells coincided temporally, it seems likely that these effects of Atr loss result directly from DNA damage. The question that arises though is why it takes so long for that damage to appear anteriorly, and why the proliferating cells of the rhombic lip are not affected while the EGL shows such a pronounced defect.

Parallel studies are currently underway in a TopBP1 conditional knock-out in which the rhombic lip is dramatically affected by E13.5 and there is clear reduction in the SOX2-positive progenitors of both the rhombic lip and the dorsal wall of V4 that produces progenitor cells which will generate cells such as cerebellar interneurons. We hope that through extensive comparison of the TopBP1 mutant and the Atr ${ }^{\text {Nes-cre }}$ mice, the relationship between ATR and TopBP1and the specific requirements for ATR activity in different cell types can be determined. Of course, we cannot neglect the possibility that Cre activity is less efficient in some cell types of the Atr $^{\text {Nes-cre }}$ embryos (despite the phenotype of the TopBP1 mutants crossed with the same Nestin-cre line). There is also the question as to how long Atr protein produced prior to gene deletion remains stable in these cell types. There is not currently a suitable antibody against Atr with which we can directly assess protein expression, so in situ hybridization experiments are underway to better evaluate these issues. Germline deletion of Atr leads to lethality by E7.5, suggesting that Atr is depleted within 24 hours after maternal supply is exhausted.

I have shown here that p53 is stabilized in the Atr-deficient nervous system and is responsible for apoptosis of cells in the EGL and VZ in response to DNA damage occurring after Atr loss. A subset of cells in the cerebellum and other developing regions 
showed Atm-dependant phosphorylation of p53 serine 18 in $A t r^{\text {Nes-cre }}$ embryos. Induction of p53 was correlated with apoptosis in the developing $A t r^{\text {Nes-cre }}$ brain. In $A t r^{\text {Nes-cre }} \mathrm{Atm}^{\text {Nes- }}$ ${ }^{c r e}$ embryos, p53 serine 18 phosphorylation was severely attenuated, but apoptosis occurred at a level consistent with that of $\mathrm{Atr}^{\text {Nes-cre }} \mathrm{Atm}^{\mathrm{Ctrl}}$ samples. Apoptosis observed in the embryonic $A t r^{N e s-c r e}$ brain is p53-dependant however, as cell death was significantly abrogated in Atr $^{\text {Nes-cre }} p 53^{-/}$animals. We speculate that the cells exhibiting Atmdependant $\mathrm{p} 53$ serine 18 phosphorylation had recently exited the cell cycle carrying damage accrued during replication, which resulted in death. However, experiments (such as co-labeling with TUNEL and antibodies against cell cycle markers or doublecortin, which marks post-mitotic, differentiating cells) have not been performed to determine if this is the case. This data also indicates a mechanism of DNA-damage induced p53 stabilization that is independent of either Atr or Atm. Further work will be necessary to identify the factor(s) responsible for p53 induction in this situation. Possibilities include DNA-PKcs, which is another serine/threonine kinase similar to ATR and ATM, but so far has been characterized primarily as a part of the NHEJ repair process (Mahaney et al., 2009). The role of DNA-PKcs in the nervous system is unclear.

Though ATR and ATM deficiency result in highly different neuropathologies, the possibility exists that there are still overlapping, redundant functions in the brain. ATR has been reported to function upstream of ATM. Fibroblasts derived from an ATR-Seckel syndrome individual showed defective ATM autophosphorylation on serine 1981 in response to either hydroxyurea-induced replication stress or UV-induced damage. Targeting ATR by siRNA was used to show normal ATM serine 1981 phosphorylation in response to IR (Myers \& Cortez, 2006; Stiff et al., 2006). Alternatively, it has also been reported that ATM and NBS1 are required for full ATR-dependent phosphorylation of Chk1 during S and G2 phases of the cell cycle and for the formation of RPA-coated ssDNA, which is required for ATRIP mediated ATR recruitment (Jazayeri et al., 2006). Additionally, it has been reported that IR-induced Chk1 phosphorylation depends upon ATR, ATM, and MRE11, but HU and UV-induced Chk1 phosphorylation is ATRdependant and independent of ATM and MRE11. It has been shown also that ATM and MRE11 are required for formation of RPA-coated ssDNA (Myers \& Cortez, 2006). While we have confirmed that Chk1 phosphorylation is indeed decreased in Atr ${ }^{\text {Nes-cre }}$ brains (data not shown), we have not adequately addressed the contribution of Atm to Chk1 activation in vivo. These studies will be performed in the near future. Thus far, we have made no attempts to link MRN activity with Atr requirements in the nervous system. This is a highly compelling idea however, particularly given the similarities between ATR-Seckel syndrome and NBS. Further evidence of potential in vivo interactions between Nbs1 and Atr might also be hinted at in the phenotypic similarities between the conditional Nbs1 knock-out mice and the Atr $^{\text {Nes-cre }}$ animals (Frappart et al., 2005).

In essence this study provides important insight into the pathology and etiology of ATR-Seckel syndrome. Based upon the microcephaly observed in the Atr ${ }^{\text {Nes-cre }}$ mice, we can extrapolate that ATR-Seckel syndrome is distinct from LIG4 syndrome in that it results from a combination of apoptosis and decreased proliferation (LIG4 syndrome appears to result strictly from apoptosis of newly post-mitotic cells in the developing 
nervous system). The data shown here also indicate that while some Atm activity is induced in the Atr ${ }^{\text {Nes-cre }}$ brain (based upon p53 serine18 phosphorylation), Atr and Atm signaling pathways operate relatively independently in the developing nervous system. Furthermore, this study also provides a more detailed account of the specificity with which ATM and ATR are required to monitor genomic integrity during different stages of the cell cycle. 


\section{CHAPTER 4 DISCUSSION}

Human syndromes in which the DNA damage response is defective can result in a variety of deleterious outcomes ranging from immunodeficiency, cancer predisposition, and infertility to profound neuropathology in the form of either degeneration or microcephaly. In particular, DNA damage poses a tremendous threat to the human nervous system, and when DNA repair mechanisms go awry the structural and functional integrity of the nervous system is at stake. Some diseases associated with DSB repair defects include ataxia-telangiectasia, Nijmegen breakage syndrome, ataxia-telangiectasialike disease, LIG4 syndrome, and ATR-Seckel syndrome. Additionally, defective singlestrand break repair mechanisms can also result in neurodegeneration similar to that of A$\mathrm{T}$, but without any extraneural deficiencies. Examples of SSB repair deficiencies include ataxia with oculomotor apraxia 1 and spinocerebellar ataxia with axonal neuropathy. Similarly, mutations in components of the nucleotide excision repair pathway have been associated with the human syndromes xeroderma pigmentosum, Cockayne syndrome, and trichothiodystrophy, all of which present with varying degrees of neuropathology. While these conditions share many phenotypic similarities, each has its own distinct characteristics attributable to the gene and repair pathway affected (McKinnon, 2009). My work has sought to determine the signaling mechanisms that prevent the neurologic phenotypes of Nijmegen breakage syndrome, ataxia-telangiectasia, ataxia-telangiectasia like disease, and ATR-Seckel syndrome.

Atm-dependent apoptosis is necessary for elimination of newly post-mitotic, genotoxically stressed cells during neurogenesis (Herzog et al., 1998). The MRN complex is required for efficient ATM activity, and deficiencies in its components Mre11 and $\mathrm{Nbs} 1$ result in immunodeficiency and radiation sensitivity similar to that seen in ATM-deficiency. However, NBS results in microcephaly while A-T and ATLD are characterized by neurodegeneration (Gatti \& Swift, 1985; McKinnon, 2009; Stewart et al., 1999; van der Burgt et al., 1996). To determine why MRE11 and Nbs1 hypomorphic mutations result in different neuropathology, we compared the DNA damage response in the nervous systems of Mrell and $\mathrm{Nbs} 1$ mutant mice with that of $\mathrm{Atm}^{-/-}$mice. The Mre $11^{A T L D 1 / A T L D 1}$ and $N b s 1^{A B / A B}$ mutant mice were designed to mimic the hypomorphic mutations identified in ATLD and NBS individuals, respectively (Theunissen et al., 2003; Williams et al., 2002). Despite decreased Atm autophosphorylation after DNA damage in the brains of both models, the $N b s 1^{\Delta B / \Delta B}$ mutants showed an otherwise normal response to IR and endogenously occurring damage incurred in Nbs1, Lig4 double deficient mice. In contrast, Mre $11^{\text {ATLDI/ATLD1 }}$ mutants showed abrogated Atm-dependent, Chk2 and p53induced apoptosis of early post-mitotic neural cells in response to DSBs, similar to $\mathrm{Atm}^{-/-}$ animals. We postulate that therein lays the distinction between ATLD and NBS neuropathology.

Similar to NBS, ATR-Seckel syndrome is characterized by microcephaly, but is associated with hypomorphic mutations in the ATR kinase (O'Driscoll et al., 2003). ATM and ATR share structural similarities as well as overlapping substrates, but respond to different forms of damage and undergo distinct modes of activation (Cimprich \& Cortez, 
2008; Mordes \& Cortez, 2008). In vitro studies indicate direct cooperation and crosstalk in both function and interaction between these molecules (Cimprich \& Cortez, 2008). Physiologically it is unclear how relevant these studies are, particularly because the neurologic consequences of ATM and ATR deficiencies are so distinct. Atr ${ }^{-/}$mice die very early in embryonic development ( E6-7.5), so a conditional Atr knock-out mouse model (Brown \& Baltimore, 2000) was used to compare the requirements for these two kinases in the nervous system. Atr ${ }^{\text {Nes-cre }}$ mice were born at a Mendelian ratio, but typically died within the first week of life. Failure to thrive and severe neurogenesis defects were deemed to be the cause of death. Decreased proliferative capacity and p53dependant apoptosis caused depletion of the cerebellar EGL and abnormal forebrain development. These characteristics of the Atr ${ }^{\text {Nes-cre }}$ animals resemble those described in ATR-SS individuals, and were due primarily to proliferation failure of neural progenitors as p53-deficiency rescued apoptosis without dramatically impacting CNS morphology. Atm-deficiency did not impact the Atr $^{\text {Nes-cre }}$ phenotype, though it did abrogate phosphorylation of p53-serine 18. Total p53 stabilization was not affected by Atm-loss and apoptosis was still detected in Atr $^{\text {Nes-cre }} \mathrm{Atm}^{\text {Nes-cre }}$ embryos. Thus, we conclude that while ATR and ATM likely cooperate in maintaining CNS integrity, the two proteins do not appear to directly interact genetically.

As a whole, this work provides deeper understanding into the requirements of ATR and the MRN complex in DSB signaling in the nervous system. Prior to these studies, most knowledge of ATR and MRN behavior was restricted to work using either primary fibroblasts or immortalized cell lines, neither of which are appropriate indicators of physiologic conditions in the nervous system. However, the mouse models used here have their limitations. While inactivating mutations in ATM and Mre11 cause neurodegeneration in humans, there is no obvious neuropathology in either $\mathrm{Atm}^{-/}$or Mre $11^{A T L D 1 / A T L D 1}$ mice. Similarly, $N b s 1^{\triangle B / \triangle B}$ mice do not exhibit striking microcephaly though there is a minor, but statistically significant reduction in brain size. While the Atm, Mre11, and Nbs1 mutants used were designed to reflect specific mutations associated with the human counterparts, the Lig $4^{\text {Nes-cre }}$ and $A t r^{\text {Nes-cre }}$ mice reflect tissuespecific deletion rather than the hypomorphic mutations reported in LIG4 syndrome and ATR-Seckel syndrome. Thus each mouse model used carries its own caveats in translating these data to the respective human conditions. However, it is only through animal studies that understanding of the neurologic impact of these diseases will ever be gained, and mice are currently the most accessible mammals in which to undertake genetic studies.

\subsection{Apoptosis Is Central to the Manifestation of Neuropathology Associated with DSB Repair Deficiencies}

The DSB response normally results in either p53-dependant apoptosis or cell cycle arrest (Vousden \& Lu, 2002). The proper balance between cellular death and proliferation is particularly important to ensure morphological and functional integrity of the nervous system. $\mathrm{Atm}^{-/-}$mice fail to induce proper p53 signaling in response to either IR or endogenously occurring DNA damage, resulting in failure of genotoxically stressed 
neural cells to undergo apoptosis (Chong et al., 2000; Gao et al., 2000; Herzog et al., 1998; Lee et al., 2000; Sekiguchi et al., 2001). This failure to induce p53-dependant apoptosis is evidence that there is a DSB signaling deficit in the $\mathrm{Atm}^{-/-}$brain, and it is postulated that cells which would otherwise have been eliminated from the developing nervous system become incorporated as mature neurons and later undergo degeneration due to genotoxic stress. It is unknown why mouse models fail to mimic the neurologic aspects of A-T. It is distinctly possible that there are genetic redundancies in the mouse brain that are lacking in humans, thus making mice more resistant to Atm loss. Another theory as to why $\mathrm{Atm}^{-/-}$mice fail to recapitulate the cerebellar ataxia and degeneration characteristic of A-T is simply that timing of cerebellar maturation and lifespan do not accommodate the length of time required for such damage to accrue and manifest. It is typically not until at least 12-14 months of age that ataxia is noted in children with A-T (Gatti \& Swift, 1985). Atm $^{-/}$mice typically die of thymic lymphomas between 2-4 months of age (Barlow et al., 1996; Elson et al., 1996), however those that evade tumors do not exhibit ataxia by 12 months of age. As with $\mathrm{Atm}^{-/-}$mice, Mre $11^{\text {ATLDI/ATLDI }}$ animals are a phenocopy of the extraneural characteristics of ATLD though there is no direct evidence of neurodegeneration or consequent ataxia (Theunissen et al., 2003). Because increased tumorigenesis is not observed in Mre $11^{\text {ATLD1/ATLD1 }}$ animals, they survive the normal murine lifespan up to 2 years, but fail to exhibit signs of neuropathology. Thus, the shorter lifespan of $\mathrm{Atm}^{-/-}$mice is still an insufficient explanation as to why neurodegeneration is not observed. Furthermore, the accumulation of $\gamma-\mathrm{H} 2 \mathrm{AX}$ and 53BP1 foci without consequent apoptosis in the adult Lig $4^{\text {Nes-cre }}$ brain demonstrates that unlike immature neurons, mature neurons are highly impervious to DNA damageinduced apoptosis. Cell cycle re-entry has been associated with death of post-mitotic neurons in neurodegenerative disorders such as Alzheimer's disease and Parkinson's disease. It has been suggested that DNA damage promotes cell cycle re-entry of postmitotic Purkinje cells in human A-T tissue samples as well as in $\mathrm{Atm}^{-/-}$and $\mathrm{Atm}^{+/-}$mice (Yang \& Herrup, 2005). In contrast, a separate study claims that Atm suppression protects post-mitotic cells from cell cycle re-entry and apoptosis in response to DNA damaging agents (Kruman et al., 2004). Most recently, Cdk5 has been implicated in activating Atm through phosphorylation of serine 794 in post-mitotic cultured granule cells. Interruption of Cdk5 signaling reportedly inhibits ATM activation (as indicated by serine 1981 autophosphorylation and phosphorylation of p53 on serine 18), and prevents DNA damage-induced cell cycle re-entry in cerebellar granule cells (Tian et al., 2009). Thus, there is conflicting data regarding whether or not ATM is required to induce neuronal cell death via cell cycle re-entry or whether ATM deficiency induces death through such a mechanism in mammalian tissues. Adequate studies of the relationship between ATM and Cdk5 have not been undertaken in vivo. Furthermore, the indication that $\mathrm{Cdk} 5$ is required for ATM activation indicates the need for further study in regard to how it and the MRN complex might cooperate in ATM activation, specifically in the nervous system.

Although the etiology of ataxia in either A-T or ATLD is not clear, both proteins are required for the DSB-induced death of early post-mitotic neural precursors through p53 signaling (Chong et al., 2000; Herzog et al., 1998; Lee et al., 2000; Lee et al., 2001; Sekiguchi et al., 2001; Shull et al., 2009). In contrast, normal p53 signaling and 
subsequent apoptosis at least partially explain the microcephaly observed in LIG4 syndrome and NBS. In the instance of LIG4 syndrome, mouse models provide a very clear cut case that unrepaired DSBs accumulate in post-mitotic neurons during embryogenesis and lead to Atm/Mre11/p53-dependent apoptosis that results in a drastic size reduction of the mature brain (Ferguson et al., 2000; Frank et al., 2000; Lee et al., 2000; Sekiguchi et al., 2001; Shull et al., 2009). The nuances of Nbs1 function in the brain are less clear, as despite its interactions with Mre11 and Atm, the impact of disrupted Nbs1 activity in the nervous system does not overlap with that of either Mre11 or Atm. In the $N b s 1^{\Delta B / \Delta B}$ mutant, a DNA repair defect likely leads to increased cell death during neurogenesis and subsequent microcephaly. The $N b s 1^{\Delta B}$ allele lacks the FHA/BRCT N-terminal domains, similar to the mutations identified in NBS individuals (Williams et al., 2002). According to our data the Nbs1 N-terminus is thus not required for apoptotic induction through Atm (Shull et al., 2009). This is not entirely surprising as Atm binds Nbs1 through its C-terminus, and this interaction is required for efficient phosphorylation of Atm targets (Falck et al., 2005). However, the physiological significance of this interaction in relation to NBS pathology is complicated. $N b s 1^{\Delta C / \Delta C}$ mutant mice were indistinguishable from $W T$ in terms of IR sensitivity and chromosomal stability (Difilippantonio et al., 2007; Stracker et al., 2007). While Stracker et al. reported defects in the intra-S-phase checkpoint; Difilippantonio et al. did not observe cell cycle checkpoint defects at any stage. Both groups observed abrogated apoptotic signaling through Atm in the thymus, but Difilippantonio et al. reported normal apoptotic induction in the nervous system of $N b s 1^{\Delta C / \Delta C}$ mutants. Thus, the connection between the Nbs1-Atm interaction and Atm signaling in the brain remains somewhat obscure. The conditionally null Nbs1 mutant $\mathrm{Nbn}^{\mathrm{f6} / \mathrm{f6}}$ mice support this conclusion, as p53-dependent apoptosis is a major contributor to the microcephaly and neurogenesis defects reported in this animal model (Frappart et al., 2005). Interestingly, NBS and ATR-Seckel syndrome share more similarities than do NBS and A-T. As already alluded to in Chapter 3, the $\mathrm{Nbn}^{\mathrm{fb} / \mathrm{f6}}$ (also on a Nestin-cre background) and Atr ${ }^{\text {Nes-cre }}$ mice have strikingly similar phenotypes that feature cerebellar dysgenesis and p53 stabilization. Unlike the Atr $^{\text {Nes-cre }}$ phenotype however, p53 deficiency largely corrected the aberrant neurogenesis of $N b n^{f 6 / 66}$ mice, suggesting that p53-dependant apoptosis was the major factor in microcephaly (Frappart et al., 2005). Based upon our data and that reported in the $N b n^{f 6 / f 6}$, we predict microcephaly associated with NBS to be the direct result of unrepaired DNA DSBs that result in p53-dependant apoptosis. Through treatment of neural stem cells isolated from $\mathrm{Nbn}^{f 6 / f 6}$ pups with the Atm-inhibitor caffeine, Frappart et al. also provided some evidence that Atm was responsible for p53 induction. However, there is currently no report of $\mathrm{Nbn}^{f 6 / \mathrm{f} 6} \mathrm{Atm}^{-/-}$mice to confirm that to be the case.

While the link to p53 signaling after DNA damage in the phenotypes of $\mathrm{Atm}^{-/}$, Mre $11^{A T L D 1 / A T L D 1}, N b s 1^{\triangle B / \triangle B}$, and Lig4-deficient mice is clear, the signaling pathway that accounts for the $A t r^{\text {Nes-cre }}$ phenotype remains to be determined. Despite the large amount of p53-dependant apoptosis observed in proliferating regions of the Atr ${ }^{\text {Nes-cre }}$ brain, p53 loss did not affect the overall phenotype. This indicates that p53-dependent apoptosis is only a minor consequence of Atr loss, and that p53 induction is not a major function of ATR in the nervous system. Though phosphorylation of p53-serine 18 was blocked in the Atr $^{\text {Nes-cre }}$ Atm $^{\text {Nes-cre }}$ animals, total p53 induction was unaffected and apoptosis was not 
dramatically attenuated. These results imply that Atm signaling through p53 was responsible for the elimination of only a small subset of Atr ${ }^{\text {Nes-cre }}$ cells, and that Atr and Atm signaling appear independent though somewhat cooperative in the nervous system. Indeed, it has been demonstrated using the $\mathrm{Xrcc}^{-/-}$animals in which p53-dependent apoptosis occurs in proliferating cells of the developing nervous system that Atm does not contribute to apoptosis resulting from HR inactivation (Orii et al., 2006). It was speculated that Atr might be the kinase responsible for stabilizing p53 in proliferating neural precursors, but that seems less likely to be the case in light of these data.

The half-life of p53 is reportedly only 10-30 minutes and many factors contribute to activation and stability of the protein (Culmsee \& Mattson, 2005; DeLeo et al., 1979). The major events involved in p53 stabilization include phosphorylation, acetylation, interaction with MDM2, and proteasome inhibition. Numerous phosphorylation sites have been identified as contributing to p53 stabilization and activity (Culmsee \& Mattson, 2005). In addition to p53-serine 18, another such site is serine 23 (the human homologue is p53-serine 20), which has been linked to the apoptotic function of p53. Studies in mice carrying p53 serine $18 / 23$ to alanine mutations indicate that p53phosphoserine 18 is only partially required for DNA damage induced apoptosis. While $p 53^{S 18 / 23 A}$ mutant mice completely rescued neural apoptosis and embryonic lethality in the $\mathrm{Xrcc}^{-/-}$genetic background, mice carrying only the S18A mutation barely impacted the $\mathrm{Xrcc}^{-/}$phenotype (Chao et al., 2006). Because the $p 53^{S 18 / 23 A}$ mutants showed much less apoptotic induction than either $p 53^{S 18 A}$ or $p 53^{S 23 A}$ mutants, it can be concluded that phosphorylation of both serine 18 and serine 23 synergistically optimize p53-dependent apoptosis in proliferating cells (Chao et al., 2003; Chao et al., 2006; MacPherson et al., 2004). One report indicates that neither ATM nor ATR phosphorylate p53 on serine 20, which would be consistent with the presence of ATM/ATR-independent, p53-dependent apoptosis observed in the Atr ${ }^{\text {Nes-cre }}$ Atm $^{\text {Nes-cre }}$ mice (Chehab et al., 1999). However, p53serine 23 is a target of Chk2 (Chehab et al., 2000). Chk2 hyperphosphorylation is severely abrogated in $\mathrm{Atm}^{-/-}$cells (Ahn et al., 2000; Falck et al., 2001; Melchionna et al., 2000). After IR, the $\mathrm{Atm}^{--}$thymus exhibits approximately half the number of viable cells as does the $p 53^{---}$thymus. However, $\mathrm{Atm}^{--} \mathrm{Chk2^{-- }}$ mice exhibit a similar amount of viable cells as the $p 53^{-/}$mice in the irradiated thymus (Stracker et al., 2007). Similarly, IRinduced apoptosis was further abrogated in $\mathrm{Mrel} 1^{A T L D 1 / A T L D 1} \mathrm{Chk2}^{-/-}$and $\mathrm{Nbs} 1^{\triangle B / \triangle B} \mathrm{Chk2} 2^{-/-}$ thymocytes (Stracker et al., 2008; Stracker \& Petrini, 2008). This suggests that Chk2 mediation of p53-induced apoptosis is only partially dependent upon Atm. The level of p53-phosphoserine 20 has not been examined in the Atr ${ }^{\text {Nes-cre }}$ mice, but IR-induced phosphorylation of Chk2 is consistent with levels observed in $\mathrm{Atr}^{\mathrm{Ctrl}}$ embryonic brains and in cultured neural stem cells (data not shown). Thus, it is necessary to perform western blot analysis of Chk2 phosphorylation to begin determining the signaling mechanism behind p53 stabilization in the developing Atr $^{\text {Nes-cre }}$ Atm $^{\text {Nes-cre }}$ brains.

Though Atr-/Atm-independent Chk2 activity is a highly compelling candidate for induction of $\mathrm{p} 53$ in the $\mathrm{Atr}^{\mathrm{Nes}-\mathrm{cre}} \mathrm{Atm}^{\mathrm{Nes}-\mathrm{cre}}$ nervous system, there are other molecules that could also be responsible. As already alluded to in Chapter 3, p63 and p73 are also implicated in IR-induced activation of p53 in the embryonic brain (Flores et al., 2002). The potential roles of Atr and Atm signaling in mediation of p63/p73-induced p53 
activity have not been explored. ARF signaling is another possible mechanism by which p53 stabilization might occur in Atr $^{\text {Nes-cre }}$ Atm $^{\text {Nes-cre embryos. The murine Ink } 4 a \text { locus }}$ encodes the tumor suppressor p19 $9^{\text {Arf }}$, which induces cell cycle arrest through upregulation of p53 expression (Kamijo et al., 1997; Quelle et al., 1995). In Atm $^{--}$MEFs, $p 19^{A R F}$ deficiency reversed premature replicative senescence though $\mathrm{Atm}^{-/-} \mathrm{ARF}^{-/-}$mice retained radiation sensitivity and lymphoma latency was unchanged (Kamijo et al., 1999). Signaling through $\mathrm{p} 19^{\text {Arf }}$ could thus be responsible for the proliferation defects observed in the $A t r^{\text {Nes-cre }}$ nervous system, but if that was the case, then the $p 53^{-/-}$background should have had a more positive effect upon neurogenesis defects in the $A t{ }^{\text {Nes-cre }}$ mice. Indeed, $A t r^{\text {Nes-cre }}$ mice were crossed onto an Ink4a/ARF mutant background, with no dramatic effect upon viability or brain morphology (data not shown). Embryonic studies have not yet been performed on these double mutants to ascertain the effects of Ink $4 a / A R F$ loss on p53 induction. Regardless of the signaling mechanisms behind p53 induction in the Atr $^{\text {Nes-cre }}$ Atm $^{\text {Nes-cre }}$ mice, it is clear that neither p53-dependent cell cycle arrest nor apoptosis are significantly responsible for the neurogenesis defects observed in the $\mathrm{Atr}^{\mathrm{Nes}-}$ ${ }^{c r e}$ mutants. This drastically separates the ATR-Seckel syndrome phenotype from the phenotypes of A-T, ATLD, NBS, and LIG4 syndrome in which p53 signaling is paramount.

\subsection{The Impact of DNA Damage Signaling upon Cell Cycle Regulation in Neural Progenitor Cells}

While the role of ATM appears to be restricted to post-mitotic cells during neurogenesis, ATR and MRN are required to ensure replication of neural progenitor cell populations. The Lig4/Xrcc4 complex is critical to the NHEJ repair pathway, which is the primary mechanism through which DSBs are repaired in post-mitotic neurons. Deficiency of either Lig4 or Xrrc4 leads to DNA damage accumulation and Atm/p53dependent apoptosis in post-mitotic cells during neurogenesis (Frank et al., 2000; Gao et al., 2000; Lee et al., 2000). Xrcc2 is essential for the HR repair pathway, which is preferred in replicating cells and germline inactivation of Xrcc 2 results in embryonic lethality and neural apoptosis (Deans et al., 2000). In contrast to findings in Lig4deficient mice, $\mathrm{Xrcc}^{-/-}$animals showed p53-dependent apoptosis in proliferating regions of the embryonic nervous system that was not Atm-dependent (Orii et al., 2006). This provided further indication that Atm is primarily involved in initiating apoptosis via p53 in post-mitotic cells during neurogenesis. In unpublished data (not shown) The Mre $11^{A T L D 1 / A T L D 1}$ genetic background had no affect upon apoptosis in the embryonic $\mathrm{XrcC}^{-/-}$nervous system, similar to the $\mathrm{Atm}^{-/-}$background (Personal communication with Dr. Youngsoo Lee, March 6, 2009). However, the $N b s 1^{\Delta B / \Delta B}$ background reduced $X r c c 2^{-/-}$ viability from the median age of E13.5 to E9.5, suggesting that Nbs1 has a potential role in HR (Personal communication with Dr. Youngsoo Lee, March 6, 2009). A role for $\mathrm{Nbs} 1$ function in ensuring replication fidelity through mediation of DNA repair is also implicated by the $\mathrm{Nbn}^{f 6 / f 6}$; Nestin-cre mutant mouse. In addition to apoptosis, proliferation was decreased in the cerebellar EGL of these mice, as indicated by BrdU labeling experiments (Frappart et al., 2005). However, it was not indicated in that report if this replication defect was related to $\mathrm{p} 53$-induced cell cycle checkpoint activation (in 
other words, was it rescued by p53 deficiency or was it p53-independent, as the proliferation decrease in the Atr ${ }^{\text {Nes-cre }}$ mutant seems to be?). Furthermore, the early embryonic lethality around E7.5 observed in $\mathrm{Nbs}^{-/-}$mice is sufficient to indicate that Nbs1 is required for HR (Zhu et al., 2001).

Similarly, conditional deletion of Mre11 in mouse embryonic stem cells revealed its requirement for proliferation (Xiao \& Weaver, 1997). Mre11 nuclease activity (which is retained in the Mre $11^{A T L D I / A T L D 1}$ mutant) has recently been implicated in HR repair as well as in contributing to ATR activation, both of which would be consistent with early embryonic lethality. Mre $11^{H 129 N / H 129 N}$ mutant mice are embryonic lethal by E10.5 due to overall failure to develop normally, it was particularly noted that there was no evidence of heart development. Loss of p53 did not impact viability. MEFs derived from a conditional allele showed decreased proliferation and genomic instability. Though this mutation did not affect ATM activity, it did confer hypersensitivity to IR and replication stress, consistent with decreased ATR activity that was indicated by very attenuated phosphorylation of Chk1 on serine 345 in Mre $11^{H 129 N / H 129 N}$ cells (Buis et al., 2008). Thus, the MRN complex has been linked in vivo to ATR and ATM signaling.

There is evidence that in addition to its interactions with ATM, the MRN complex is required for maintaining replication fidelity through association with ATR. Nbs1 activity has been implicated both upstream and downstream of ATR signaling. Nbs1 is phosphorylated on serine 343 by ATR, in response to stalled replication forks (Olson et al., 2007a). Hyperphosphorylation of RPA in response to hydroxyurea treatment requires ATR, but phosphorylated Nbs1 is required for this activity independently of ATM activity (Manthey et al., 2007). It has also been shown that the MRN complex interacts with ATRIP, a factor required for ATR activation, through the FHA/BRCT domains of Nbs1 (Olson et al., 2007a). Interaction of the MRN complex with RPA also occurs through the Mre11 C-terminus and is CDK-dependent (Olson et al., 2007b). Nbs1 reportedly interacts physically with TopBP1, which is involved in ATR activation through association with ATRIP, and its expression is reportedly decreased in Nbs1deficient cells (Mordes et al., 2008; Morishima et al., 2007). Consistent with requirements for Nbs1 upstream of ATR signaling, NBS cells reportedly show diminished phosphorylation of ATR targeted regions on Chk1, c-Jun, and p53 in response to either UV-irradiation or hydroxyurea treatment (Stiff et al., 2005). Another potential connection between ATR activity and MRN exists through the CtIP protein. The yeast homologue to human CtIP is Ctp1, which is required for efficient formation of RPAcoated ssDNA adjacent to DSB sites (Limbo et al., 2007). Human CtIP is recruited to DSB sites exclusively during S/G1 phases and interacts with the MRN complex. CtIP is required for DSB end resection and thus for RPA and ATR recruitment to the break site (Sartori et al., 2007). Though more work is required to firmly establish the relationship between the MRN complex and ATR, it is a very compelling notion that these molecules are functionally entwined, particularly in light of their common developmental requirements and the similar consequences of hypomorphic mutations associated with NBS and ATR-SS. 
ATR signaling through claspin/Chk1 is critical to both the DNA damage response and controlling replication (Cimprich \& Cortez, 2008). Through Rad17 modulation, claspin acts as an adaptor molecule at the replication fork to facilitate the ATR-Chk1 interaction, following TopBP1-ATRIP mediated ATR activation (Cimprich \& Cortez, 2008; Kumagai \& Dunphy, 2000; Liu et al., 2006; Shiotani \& Zou, 2009; Wang et al., 2006). Once activated by ATR, Chk1 is released from chromatin and free to phosphorylate its own substrates such as the CDC25 phosphatases, which regulate cell cycle progression (Cimprich \& Cortez, 2008; Smits et al., 2006). The human genome contains 3 CDC25 genes, each of which is subject to alternative splicing. The CDC25 phosphatases regulate cell cycle transitions through dephosphorylating and thereby activating cyclin dependent kinases (CDKs). All three isoforms (CDC25 A-C) are involved in regulating the G1-S and G2-M transitions, as well as mitosis. During S-phase, the inactivation of CDC25A is mediated by DNA damage-induced Chk1 and Chk2 activity. G1 arrest is induced in response to DNA damage when Chk1 phosphorylates CDC25A, which results in its degradation and subsequent $\mathrm{G} 2$ arrest. In response to IR, Chk1 and Chk2 phosphorylate CDC25C, which prevents its interaction with CDK1cyclin B. CDC25B is also reportedly phosphorylated by Chk1, resulting in G2/M arrest. In addition to ATM/ATR mediated CDC25 phosphorylation, p38 also regulates checkpoint activation through CDC25 phosphorylation (Boutros et al., 2006).

The impact of ATR and ATM inactivation upon CDC25 and subsequent cyclin mediation has not been thoroughly characterized in the nervous system. Cells isolated from individuals with either ATR-SS or primary microcephaly caused by mutations in the microcephalin/BRIT1 gene (MCPH1) showed reduced CDC15A degradation associated with failure to activate the $\mathrm{G} 2 / \mathrm{M}$ checkpoint, increased percentage of cells showing nuclear fragmentation, and an increased number of cells exhibiting supernumerary mitotic centrosomes in response to DNA damage (Alderton et al., 2006). Chk2 is a major ATM substrate, and in vitro ATM-deficiency results in G1-S and G2-M checkpoint failure, as well as early cellular senescence attributed to decreased p53 and Chk2 activity (Lavin, 2008). In the case of ATR and Nbs1 function in the nervous system, we can speculate that decreased cell cycle checkpoint activation results in aberrant replication followed by senescence or apoptosis of cells that have incurred genotoxic levels of DNA damage, hence the observations of apoptosis and decreased proliferation observed in the

developing brains of $\mathrm{Atr}^{\mathrm{Nes}-\mathrm{cre}}$ and $\mathrm{Nbn}^{f 6 / f 6}$ mutant mice (Frappart et al., 2005). Despite the observations of cell cycle checkpoint defects and early senescence in cells cultured from ATM deficient humans and mice, as well as from ATLD individuals and the Mre $11^{A T L D 1 / A T L D 1}$ mutant animals, the data presented here provides no indication that either inactivating ATM mutations or the hypomorphic mutations associated with ATLD have any impact upon cell cycle checkpoint integrity or proliferation in the nervous system (Lavin, 2008; Stewart et al., 1999; Taylor et al., 2004; Theunissen et al., 2003).

\subsection{Concluding Remarks}

ATM, ATR, and the MRN complex are integral components of the DNA DSB signaling machinery. Autosomal recessive genetic mutations in ATM, ATR, Mre11, and 
Nbs1 have been linked to diseases that share many characteristics such as cell cycle checkpoint deficiencies in cell lines such as fibroblasts, immunodeficiency, increased radiation sensitivity, and genomic instability. Inactivating, autosomal recessive mutations in ATM are responsible for ataxia-telangiectasia, which is characterized by neurodegeneration and consequent ataxia, cancer proneness, ocular telangiectasias, increased $\alpha$-fetoprotein levels, and immunodeficiency (OMIM \#208900). Hypomorphic mutations in ATR have been identified in a subset of individuals diagnosed with Seckel syndrome (OMIM \#601215). Seckel syndrome presents with intrauterine growth defects, microcephaly, dwarfism, and variable degrees of mental retardation (OMIM \#210600). ATR-Seckel syndrome individuals present with these characteristics as well as the above mentioned hallmarks of genomic instability. ATM and ATR are similar PIKKs that divide the DNA DSB response between signal transduction of DSBs induced by sources such as IR and DSBs that result from replication stress, respectively. The MRN complex is implicated in modulating the activity of both molecules and in directly facilitating DSB repair through tethering of broken ends and 5'-3' resection (Cimprich \& Cortez, 2008; McKinnon, 2009). Hypomorphic mutations in its components Mre11 and Nbs1 result in ataxia-telangiectasia-like disease (OMIM \#604391) and Nijmegen breakage syndrome (OMIM \#251260), respectively. ATLD differs from A-T in the absence of telangiectasia and increased $\alpha$-fetoprotein levels, but presents with very similar neurodegeneration and ataxia, but with longer latency. NBS shares many characteristics with ATR-SS, particularly microcephaly.

This work has sought to delineate the signaling mechanisms that account for the distinct neuropathology observed in these individual genetic deficiencies using mouse models. While $\mathrm{Atm}^{-/-}$and $\mathrm{Mrel} 1^{\text {ATLDI/ATLDI }}$ mice show abrogated p53-dependent apoptotic induction in post-mitotic regions of the developing brain, $N b s 1^{1 B / \triangle B}$ animals showed normal levels of cell death in response to DNA DSBs despite reduced Atm activity. This data indicates that while reduced elimination of cells harboring DNA damage is involved in the pathology of A-T and ATLD, the microcephaly associated with NBS is likely attributable to increased Atm/p53-dependent cell death as a result of DNA repair deficiency. In terms of Atm signaling in response to DSBs, these results indicate that there is an as yet undefined threshold of Atm activity required to efficiently induce apoptosis and that the $N b s 1^{\Delta B / A B}$ mutation does not suppress Atm function to the same extent that the ATLD mutation does, thus producing different neurologic consequences when challenged by DNA damage (Shull et al., 2009). By comparing the neuropathology and neural DNA damage response of Atr $^{\text {Nes-cre }}$ and Atr $^{\text {Nes-cre }}$ Atm $^{\text {Nes-cre }}$ mice, we have reiterated that Atm is not significantly involved in the DNA damage response of replicating neural precursors and Atr deficiency results in microcephaly and neurogenesis defects through a combination of apoptosis and decreased proliferation of neural precursor cells associated with increased DNA damage in proliferating regions of the developing brain. While apoptotic signaling proved to be p53-dependent and Atmindependent, the signaling mechanisms behind the proliferation defect in the Atr $^{\text {Nes-cre }}$ brain have yet to be identified. Overall, the data presented here provides valuable insights into the tissue-specific requirements of ATM, ATR, and MRN signaling and sheds new light onto the etiology of the neurologic phenotypes resulting from deficiency of these molecules. 


\section{LIST OF REFERENCES}

Adams KE, Medhurst AL, Dart DA and Lakin ND. (2006). Oncogene, 25, 3894-904.

Ahn JY, Schwarz JK, Piwnica-Worms H and Canman CE. (2000). Cancer Res, 60, 59346.

Ahnesorg P, Smith P and Jackson SP. (2006). Cell, 124, 301-13.

Alderton GK, Galbiati L, Griffith E, Surinya KH, Neitzel H, Jackson AP, Jeggo PA and O'Driscoll M. (2006). Nat Cell Biol, 8, 725-33.

Audebert M, Salles B and Calsou P. (2004). J Biol Chem, 279, 55117-55126.

Bakkenist CJ and Kastan MB. (2003). Nature, 421, 499-506.

Ball HL, Ehrhardt MR, Mordes DA, Glick GG, Chazin WJ and Cortez D. (2007). Mol Cell Biol, 27, 3367-77.

Banin S, Moyal L, Shieh S, Taya Y, Anderson CW, Chessa L, Smorodinsky NI, Prives C, Reiss Y, Shiloh Y and Ziv Y. (1998). Science, 281, $1674-7$.

Barlow C, Hirotsune S, Paylor R, Liyanage M, Eckhaus M, Collins F, Shiloh Y, Crawley JN, Ried T, Tagle D and Wynshaw-Boris A. (1996). Cell, 86, 159-71.

Bartkova J, Horejsi Z, Koed K, Kramer A, Tort F, Zieger K, Guldberg P, Sehested M, Nesland JM, Lukas C, Orntoft T, Lukas J and Bartek J. (2005). Nature, 434, 86470.

Bartkova J, Rezaei N, Liontos M, Karakaidos P, Kletsas D, Issaeva N, Vassiliou LV, Kolettas E, Niforou K, Zoumpourlis VC, Takaoka M, Nakagawa H, Tort F, Fugger K, Johansson F, Sehested M, Andersen CL, Dyrskjot L, Orntoft T, Lukas J, Kittas C, Helleday T, Halazonetis TD, Bartek J and Gorgoulis VG. (2006). Nature, 444, 633-7.

Bassing CH and Alt FW. (2004). DNA Repair (Amst), 3, 781-96.

Bekker-Jensen S, Lukas C, Melander F, Bartek J and Lukas J. (2005). J Cell Biol, 170, 201-11.

Ben-Arie N, Bellen HJ, Armstrong DL, McCall AE, Gordadze PR, Guo Q, Matzuk MM and Zoghbi HY. (1997). Nature, 390, 169-72.

Bermudez VP, Lindsey-Boltz LA, Cesare AJ, Maniwa Y, Griffith JD, Hurwitz J and Sancar A. (2003). Proc Natl Acad Sci U S A, 100, 1633-8. 
Boutros R, Dozier C and Ducommun B. (2006). Curr Opin Cell Biol, 18, 185-91.

Brown EJ and Baltimore D. (2000). Genes Dev, 14, 397-402.

Brown EJ and Baltimore D. (2003). Genes Dev, 17, 615-28.

Buck D, Malivert L, de Chasseval R, Barraud A, Fondaneche MC, Sanal O, Plebani A, Stephan JL, Hufnagel M, le Deist F, Fischer A, Durandy A, de Villartay JP and Revy P. (2006). Cell, 124, 287-99.

Buis J, Wu Y, Deng Y, Leddon J, Westfield G, Eckersdorff M, Sekiguchi JM, Chang S and Ferguson DO. (2008). Cell, 135, 85-96.

Burma S and Chen DJ. (2004). DNA Repair (Amst), 3, 909-18.

Canman CE, Lim DS, Cimprich KA, Taya Y, Tamai K, Sakaguchi K, Appella E, Kastan MB and Siliciano JD. (1998). Science, 281, 1677-9.

Carletti B and Rossi F. (2008). Neuroscientist, 14, 91-100.

Celeste A, Difilippantonio S, Difilippantonio MJ, Fernandez-Capetillo O, Pilch DR, Sedelnikova OA, Eckhaus M, Ried T, Bonner WM and Nussenzweig A. (2003). Cell, 114, 371-83.

Celeste A, Petersen S, Romanienko PJ, Fernandez-Capetillo O, Chen HT, Sedelnikova OA, Reina-San-Martin B, Coppola V, Meffre E, Difilippantonio MJ, Redon C, Pilch DR, Olaru A, Eckhaus M, Camerini-Otero RD, Tessarollo L, Livak F, Manova K, Bonner WM, Nussenzweig MC and Nussenzweig A. (2002). Science, 296, 922-7.

Chanoux RA, Yin B, Urtishak KA, Asare A, Bassing CH and Brown EJ. (2008). J Biol Chem.

Chao C, Hergenhahn M, Kaeser MD, Wu Z, Saito S, Iggo R, Hollstein M, Appella E and Xu Y. (2003). J Biol Chem, 278, 41028-33.

Chao C, Herr D, Chun J and Xu Y. (2006). Embo J, 25, 2615-22.

Chehab NH, Malikzay A, Appel M and Halazonetis TD. (2000). Genes Dev, 14, 278-88.

Chehab NH, Malikzay A, Stavridi ES and Halazonetis TD. (1999). Proc Natl Acad Sci U $S A, 96,13777-82$.

Chong MJ, Murray MR, Gosink EC, Russell HR, Srinivasan A, Kapsetaki M, Korsmeyer SJ and McKinnon PJ. (2000). Proc Natl Acad Sci U S A, 97, 889-94. 
Cimprich KA and Cortez D. (2008). Nat Rev Mol Cell Biol, 9, 616-27.

Conti C, Seiler JA and Pommier Y. (2007). Cell Cycle, 6, 2760-2767.

Corbin JG, Gaiano N, Juliano SL, Poluch S, Stancik E and Haydar TF. (2008). $J$ Neurochem, 106, 2272-87.

Cortez D, Guntuku S, Qin J and Elledge SJ. (2001). Science, 294, 1713-6.

Costanzo V, Shechter D, Lupardus PJ, Cimprich KA, Gottesman M and Gautier J. (2003). Mol Cell, 11, 203-13.

Critchlow SE, Bowater RP and Jackson SP. (1997). Curr Biol, 7, 588-98.

Cuadrado M, Martinez-Pastor B, Murga M, Toledo LI, Gutierrez-Martinez P, Lopez E and Fernandez-Capetillo O. (2006). J Exp Med, 203, 297-303.

Culmsee C and Mattson MP. (2005). Biochem Biophys Res Commun, 331, 761-77.

Daniel JA, Pellegrini M, Lee JH, Paull TT, Feigenbaum L and Nussenzweig A. (2008). $J$ Cell Biol, 183, 777-83.

Deans B, Griffin CS, Maconochie M and Thacker J. (2000). Embo J, 19, 6675-85.

DeLeo AB, Jay G, Appella E, Dubois GC, Law LW and Old LJ. (1979). Proc Natl Acad Sci U S A, 76, 2420-4.

Di Micco R, Fumagalli M, Cicalese A, Piccinin S, Gasparini P, Luise C, Schurra C, Garre M, Nuciforo PG, Bensimon A, Maestro R, Pelicci PG and d'Adda di Fagagna F. (2006). Nature, 444, 638-42.

DiBiase SJ, Zeng Z-C, Chen R, Hyslop T, Curran WJ, Jr. and Iliakis G. (2000). Cancer Res, 60, 1245-1253.

Difilippantonio S, Celeste A, Fernandez-Capetillo O, Chen HT, Reina San Martin B, Van Laethem F, Yang YP, Petukhova GV, Eckhaus M, Feigenbaum L, Manova K, Kruhlak M, Camerini-Otero RD, Sharan S, Nussenzweig M and Nussenzweig A. (2005). Nat Cell Biol, 7, 675-85.

Difilippantonio S, Celeste A, Kruhlak MJ, Lee Y, Difilippantonio MJ, Feigenbaum L, Jackson SP, McKinnon PJ and Nussenzweig A. (2007). J Exp Med, 204, 1003-11.

Dornan D, Shimizu H, Mah A, Dudhela T, Eby M, O'Rourke K, Seshagiri S and Dixit VM. (2006). Science, 313, 1122-6.

Edwards MA, Yamamoto M and Caviness VS, Jr. (1990). Neuroscience, 36, 121-44. 
Ellison V and Stillman B. (2003). PLoS Biol, 1, E33.

Elson A, Wang Y, Daugherty CJ, Morton CC, Zhou F, Campos-Torres J and Leder P. (1996). Proc Natl Acad Sci U S A, 93, 13084-13090.

Falck J, Coates J and Jackson SP. (2005). Nature, 434, 605-11.

Falck J, Lukas C, Protopopova M, Lukas J, Selivanova G and Bartek J. (2001). Oncogene, 20, 5503-10.

Ferguson DO, Sekiguchi JM, Chang S, Frank KM, Gao Y, DePinho RA and Alt FW. (2000). Proc Natl Acad Sci U S A, 97, 6630-3.

Fernandez-Capetillo O, Lee A, Nussenzweig M and Nussenzweig A. (2004). DNA Repair (Amst), 3, 959-67.

Flores ER, Tsai KY, Crowley D, Sengupta S, Yang A, McKeon F and Jacks T. (2002). Nature, 416, 560-4.

Forni PE, Scuoppo C, Imayoshi I, Taulli R, Dastru W, Sala V, Betz UA, Muzzi P, Martinuzzi D, Vercelli AE, Kageyama R and Ponzetto C. (2006). J Neurosci, 26, 9593-602.

Frank KM, Sharpless NE, Gao Y, Sekiguchi JM, Ferguson DO, Zhu C, Manis JP, Horner J, DePinho RA and Alt FW. (2000). Mol Cell, 5, 993-1002.

Frappart PO and McKinnon PJ. (2006). Neuromolecular Med, 8, 495-512.

Frappart PO, Tong WM, Demuth I, Radovanovic I, Herceg Z, Aguzzi A, Digweed M and Wang ZQ. (2005). Nat Med, 11, 538-44.

Friedberg EC and Meira LB. (2006). DNA Repair (Amst), 5, 189-209.

Gao Y, Ferguson DO, Xie W, Manis JP, Sekiguchi J, Frank KM, Chaudhuri J, Horner J, DePinho RA and Alt FW. (2000). Nature, 404, 897-900.

Gatti RA and Swift M. (1985). Ataxia-telangiectasia: Genetics, Neuropathology, and Immunology of a Degenerative Disease of Childhood. Alan R. Liss: New York.

Goldberg M, Stucki M, Falck J, D'Amours D, Rahman D, Pappin D, Bartek J and Jackson SP. (2003). Nature, 421, 952-6.

Goldowitz D and Hamre K. (1998). Trends Neurosci, 21, 375-82.

Goodrich LV, Milenkovic L, Higgins KM and Scott MP. (1997). Science, 277, 1109-13. 
Grawunder U, Wilm M, Wu X, Kulesza P, Wilson TE, Mann M and Lieber MR. (1997). Nature, 388, 492-5.

Grawunder U, Zimmer D, Fugmann S, Schwarz K and Lieber MR. (1998a). Mol Cell, 2, 477-84.

Grawunder U, Zimmer D, Kulesza P and Lieber MR. (1998b). J Biol Chem, 273, 2470814.

Hallonet ME and Le Douarin NM. (1993). Eur J Neurosci, 5, 1145-55.

Herzog KH, Chong MJ, Kapsetaki M, Morgan JI and McKinnon PJ. (1998). Science, 280, 1089-91.

Hurley PJ and Bunz F. (2007). Cell Cycle, 6, 414-7.

Ito K, Hirao A, Arai F, Matsuoka S, Takubo K, Hamaguchi I, Nomiyama K, Hosokawa K, Sakurada K, Nakagata N, Ikeda Y, Mak TW and Suda T. (2004). Nature, 431, 997-1002.

Jazayeri A, Falck J, Lukas C, Bartek J, Smith GC, Lukas J and Jackson SP. (2006). Nat Cell Biol, 8, 37-45.

Jeggo P and O'Neill P. (2002). DNA Repair (Amst), 1, 771-7.

Jensen P, Zoghbi HY and Goldowitz D. (2002). J Neurosci, 22, 8110-6.

Kamijo T, van de Kamp E, Chong MJ, Zindy F, Diehl JA, Sherr CJ and McKinnon PJ. (1999). Cancer Res, 59, $2464-9$.

Kamijo T, Zindy F, Roussel MF, Quelle DE, Downing JR, Ashmun RA, Grosveld G and Sherr CJ. (1997). Cell, 91, 649-59.

Kang J, Bronson RT and Xu Y. (2002). Embo J, 21, 1447-55.

Kastan MB and Bartek J. (2004). Nature, 432, 316-23.

Kastan MB, Zhan Q, El-Deiry WS, Carrier F, Jacks T, Walsh WV, Plunkett BS, Vogelstein B and Fornace AJ. (1992). Cell, 71, 587-597.

Katyal S and McKinnon PJ. (2007). Cell Cycle, 6, 2360-5.

Khanna KK, Keating KE, Kozlov S, Scott S, Gatei M, Hobson K, Taya Y, Gabrielli B, Chan D, Lees-Miller SP and Lavin MF. (1998). Nat Genet, 20, 398-400. 
Kitagawa R, Bakkenist CJ, McKinnon PJ and Kastan MB. (2004). Genes Dev, 18, 142338.

Kobayashi J, Antoccia A, Tauchi H, Matsuura S and Komatsu K. (2004). DNA Repair (Amst), 3, 855-61.

Kozlov SV, Graham ME, Peng C, Chen P, Robinson PJ and Lavin MF. (2006). Embo J, 25, 3504-14.

Kruman, II, Wersto RP, Cardozo-Pelaez F, Smilenov L, Chan SL, Chrest FJ, Emokpae R, Jr., Gorospe M and Mattson MP. (2004). Neuron, 41, 549-61.

Kuhne M, Riballo E, Rief N, Rothkamm K, Jeggo PA and Lobrich M. (2004). Cancer Res, 64, 500-8.

Kumagai A and Dunphy WG. (2000). Mol Cell, 6, 839-49.

Kumagai A, Lee J, Yoo HY and Dunphy WG. (2006). Cell, 124, 943-55.

Lavin MF. (2008). Nat Rev Mol Cell Biol, 9, 759-69.

Lavin MF and Kozlov S. (2007). Cell Cycle, 6, 931-942.

Lee JH and Paull TT. (2004). Science, 304, 93-6.

Lee Y, Barnes DE, Lindahl T and McKinnon PJ. (2000). Genes Dev, 14, 2576-80.

Lee Y, Chong MJ and McKinnon PJ. (2001). J Neurosci, 21, 6687-93.

Lees-Miller SP and Meek K. (2003). Biochimie, 85, 1161-73.

Lieber MR, Ma Y, Pannicke U and Schwarz K. (2003). Nat Rev Mol Cell Biol, 4, 712-20.

Limbo O, Chahwan C, Yamada Y, de Bruin RA, Wittenberg C and Russell P. (2007). Mol Cell, 28, 134-46.

Liu S, Bekker-Jensen S, Mailand N, Lukas C, Bartek J and Lukas J. (2006). Mol Cell Biol, 26, 6056-64.

Lledo PM, Merkle FT and Alvarez-Buylla A. (2008). Trends Neurosci, 31, 392-400.

Lou Z, Minter-Dykhouse K, Franco S, Gostissa M, Rivera MA, Celeste A, Manis JP, van Deursen J, Nussenzweig A, Paull TT, Alt FW and Chen J. (2006). Mol Cell, 21, 187-200.

Lou Z, Minter-Dykhouse K, Wu X and Chen J. (2003). Nature, 421, 957-61. 
Lukas J, Lukas C and Bartek J. (2004). DNA Repair (Amst), 3, 997-1007.

MacPherson D, Kim J, Kim T, Rhee BK, Van Oostrom CT, DiTullio RA, Venere M, Halazonetis TD, Bronson R, De Vries A, Fleming M and Jacks T. (2004). Embo $J$, 23, 3689-99.

Mahaney BL, Meek K and Lees-Miller SP. (2009). Biochem J., 417, 639-50.

Manthey KC, Opiyo S, Glanzer JG, Dimitrova D, Elliott J and Oakley GG. (2007). J Cell Sci, 120, 4221-9.

Martinez S, Wassef M and Alvarado-Mallart RM. (1991). Neuron, 6, 971-81.

Matsuoka S, Ballif BA, Smogorzewska A, McDonald ER, 3rd, Hurov KE, Luo J, Bakalarski CE, Zhao Z, Solimini N, Lerenthal Y, Shiloh Y, Gygi SP and Elledge SJ. (2007). Science, 316, 1160-6.

McKinnon PJ. (2009). Nat Rev Neurosci, 10, 100-12.

McKinnon PJ and Caldecott KW. (2007). Annu Rev Genomics Hum Genet, 8, 37-55.

Melchionna R, Chen XB, Blasina A and McGowan CH. (2000). Nat Cell Biol, 2, 762-5.

Miale IL and Sidman RL. (1961). Exp Neurol, 4, 277-96.

Morales D and Hatten ME. (2006). J Neurosci, 26, 12226-36.

Mordes DA and Cortez D. (2008). Cell Cycle, 7, 2809-12.

Mordes DA, Glick GG, Zhao R and Cortez D. (2008). Genes Dev, 22, 1478-89.

Morishima K, Sakamoto S, Kobayashi J, Izumi H, Suda T, Matsumoto Y, Tauchi H, Ide H, Komatsu K and Matsuura S. (2007). Biochem Biophys Res Commun, 362, 8729.

Moshous D, Callebaut I, de Chasseval R, Corneo B, Cavazzana-Calvo M, Le Deist F, Tezcan I, Sanal O, Bertrand Y, Philippe N, Fischer A and de Villartay JP. (2001). Cell, 105, 177-86.

Myers JS and Cortez D. (2006). J Biol Chem, 281, 9346-50.

Nicolis SK. (2007). Neurobiol Dis, 25, 217-29.

Nijnik A, Woodbine L, Marchetti C, Dawson S, Lambe T, Liu C, Rodrigues NP, Crockford TL, Cabuy E, Vindigni A, Enver T, Bell JI, Slijepcevic P, Goodnow CC, Jeggo PA and Cornall RJ. (2007). Nature, 447, 686-90. 
O'Driscoll M, Ruiz-Perez VL, Woods CG, Jeggo PA and Goodship JA. (2003). Nat Genet, 33, 497-501.

Olson E, Nievera CJ, Lee AY, Chen L and Wu X. (2007a). J Biol Chem, 282, 22939-52.

Olson E, Nievera CJ, Liu E, Lee AY, Chen L and Wu X. (2007b). Mol Cell Biol, 27, 6053-67.

Orii KE, Lee Y, Kondo N and McKinnon PJ. (2006). Proc Natl Acad Sci U S A, 103, 10017-22.

Pabla N, Huang S, Mi QS, Daniel R and Dong Z. (2008). J Biol Chem, 283, 6572-83.

Pellegrini M, Celeste A, Difilippantonio S, Guo R, Wang W, Feigenbaum L and Nussenzweig A. (2006). Nature, 443, 222-5.

Perrault R, Wang H, Wang M, Rosidi B and Iliakis G. (2004). J Cell Biochem, 92, 781794.

Petrini JH and Stracker TH. (2003). Trends Cell Biol, 13, 458-62.

Phillips ER and McKinnon PJ. (2007). Oncogene, 26, 7799-808.

Quelle DE, Zindy F, Ashmun RA and Sherr CJ. (1995). Cell, 83, 993-1000.

Rass U, Ahel I and West SC. (2007). Cell, 130, 991-1004.

Riballo E, Critchlow SE, Teo SH, Doherty AJ, Priestley A, Broughton B, Kysela B, Beamish H, Plowman N, Arlett CF, Lehmann AR, Jackson SP and Jeggo PA. (1999). Curr Biol, 9, 699-702.

Rooney S, Sekiguchi J, Zhu C, Cheng HL, Manis J, Whitlow S, DeVido J, Foy D, Chaudhuri J, Lombard D and Alt FW. (2002). Mol Cell, 10, 1379-1390.

Rosenthal N and Brown S. (2007). Nat Cell Biol, 9, 993-9.

Ruzankina Y, Pinzon-Guzman C, Asare A, Ong T, Pontano L, Cotsarelis G, Zediak VP, Velez M, Bhandoola A and Brown EJ. (2007). Cell Stem Cell, 1, 113-26.

Sartori AA, Lukas C, Coates J, Mistrik M, Fu S, Bartek J, Baer R, Lukas J and Jackson SP. (2007). Nature, 450, 509-14.

Sedelnikova OA, Pilch DR, Redon C and Bonner WM. (2003). Cancer Biol Ther, 2, 2335. 
Sekiguchi J, Ferguson DO, Chen HT, Yang EM, Earle J, Frank K, Whitlow S, Gu Y, Xu Y, Nussenzweig A and Alt FW. (2001). Proc Natl Acad Sci U S A, 98, 3243-8.

Shiloh Y. (2006). Trends Biochem Sci, 31, 402-10.

Shiotani B and Zou L. (2009). J Cell Sci, 122, 301-4.

Shull ER, Lee Y, Nakane H, Stracker TH, Zhao J, Russell HR, Petrini JH and McKinnon PJ. (2009). Genes Dev, 23, 171-80.

Sillitoe RV and Joyner AL. (2007). Annu Rev Cell Dev Biol, 23, 549-77.

Smeyne RJ, Chu T, Lewin A, Bian F, Sanlioglu S, Kunsch C, Lira SA and Oberdick J. (1995). Mol Cell Neurosci, 6, 230-51.

Smits VA, Reaper PM and Jackson SP. (2006). Curr Biol, 16, 150-9.

Stewart GS, Maser RS, Stankovic T, Bressan DA, Kaplan MI, Jaspers NG, Raams A, Byrd PJ, Petrini JH and Taylor AM. (1999). Cell, 99, 577-87.

Stewart GS, Wang B, Bignell CR, Taylor AM and Elledge SJ. (2003). Nature, 421, 9616.

Stiff T, Reis C, Alderton GK, Woodbine L, O'Driscoll M and Jeggo PA. (2005). Embo J, 24, 199-208.

Stiff T, Walker SA, Cerosaletti K, Goodarzi AA, Petermann E, Concannon P, O'Driscoll $\mathrm{M}$ and Jeggo PA. (2006). Embo J, 25, 5775-82.

Stracker TH, Couto SS, Cordon-Cardo C, Matos T and Petrini JH. (2008). Mol Cell, 31, 21-32.

Stracker TH, Morales M, Couto SS, Hussein H and Petrini JH. (2007). Nature, 447, 21821.

Stracker TH and Petrini JH. (2008). Cell Cycle, 7, 3618-21.

Stucki M, Clapperton JA, Mohammad D, Yaffe MB, Smerdon SJ and Jackson SP. (2005). Cell, 123, 1213-26.

Stucki M and Jackson SP. (2006). DNA Repair (Amst), 5, 534-43.

Takahashi A and Ohnishi T. (2005). Cancer Lett, 229, 171-9. 
Takai H, Naka K, Okada Y, Watanabe M, Harada N, Saito S, Anderson CW, Appella E, Nakanishi M, Suzuki H, Nagashima K, Sawa H, Ikeda K and Motoyama N. (2002). Embo J, 21, 5195-5205.

Takubo K, Ohmura M, Azuma M, Nagamatsu G, Yamada W, Arai F, Hirao A and Suda T. (2008). Cell Stem Cell, 2, 170-82.

Tauchi H, Kobayashi J, Morishima K, van Gent DC, Shiraishi T, Verkaik NS, vanHeems D, Ito E, Nakamura A, Sonoda E, Takata M, Takeda S, Matsuura S and Komatsu K. (2002). Nature, 420, 93-8.

Taylor AM, Groom A and Byrd PJ. (2004). DNA Repair (Amst), 3, 1219-25.

Theunissen JW, Kaplan MI, Hunt PA, Williams BR, Ferguson DO, Alt FW and Petrini JH. (2003). Mol Cell, 12, 1511-23.

Tian B, Yang Q and Mao Z. (2009). Nat Cell Biol.

Tibbetts RS, Brumbaugh KM, Williams JM, Sarkaria JN, Cliby WA, Shieh SY, Taya Y, Prives C and Abraham RT. (1999). Genes Dev, 13, 152-7.

Traiffort E, Charytoniuk DA, Faure H and Ruat M. (1998). J Neurochem, 70, 1327-30.

van der Burgt I, Chrzanowska KH, Smeets D and Weemaes C. (1996). J Med Genet, 33, 153-6.

Vousden KH and Lu X. (2002). Nat Rev Cancer, 2, 594-604.

Wang H, Rosidi B, Perrault R, Wang M, Zhang L, Windhofer F and Iliakis G. (2005). Cancer Res, 65, 4020-4030.

Wang VY and Zoghbi HY. (2001). Nat Rev Neurosci, 2, 484-91.

Wang X, Zou L, Lu T, Bao S, Hurov KE, Hittelman WN, Elledge SJ and Li L. (2006). Mol Cell, 23, 331-41.

Williams BR, Mirzoeva OK, Morgan WF, Lin J, Dunnick W and Petrini JH. (2002). Curr Biol, 12, 648-53.

Windhofer F, Wu W, Wang M, Singh SK, Saha J, Rosidi B and Iliakis G. (2007). Int J Radiat Oncol Biol Phys, 68, 1462-70.

Wingate RJ. (2001). Curr Opin Neurobiol, 11, 82-8.

Wonders C and Anderson SA. (2005). Neuroscientist, 11, 199-205. 
Xiao Y and Weaver DT. (1997). Nucleic Acids Res, 25, 2985-91.

Yang Y and Herrup K. (2005). J Neurosci, 25, 2522-9.

Yoo HY, Kumagai A, Shevchenko A, Shevchenko A and Dunphy WG. (2007). J Biol Chem, 282, 17501-6.

You Z, Chahwan C, Bailis J, Hunter T and Russell P. (2005). Mol Cell Biol, 25, 5363-79.

Zappone MV, Galli R, Catena R, Meani N, De Biasi S, Mattei E, Tiveron C, Vescovi AL, Lovell-Badge R, Ottolenghi S and Nicolis SK. (2000). Development, 127, 236782.

Zhu J, Petersen S, Tessarollo L and Nussenzweig A. (2001). Curr Biol, 11, 105-9.

Ziv Y, Bielopolski D, Galanty Y, Lukas C, Taya Y, Schultz DC, Lukas J, Bekker-Jensen S, Bartek J and Shiloh Y. (2006). Nat Cell Biol, 8, 870-6.

Zou L and Elledge SJ. (2003). Science, 300, 1542-8.

Zou L, Liu D and Elledge SJ. (2003). Proc Natl Acad Sci U S A, 100, 13827-32. 


\section{VITA}

Erin Shull was born Erin Renee Phillips in Rockford, Illinois on June 19, 1980. She graduated from the University of Tennessee in Knoxville cum laude with a Bachelor of Science in Animal Science in 2003, and then enrolled in the Interdisciplinary program at the University of Tennessee Health Science Center. 